Provided for non-commercial research and education use. Not for reproduction, distribution or commercial use.

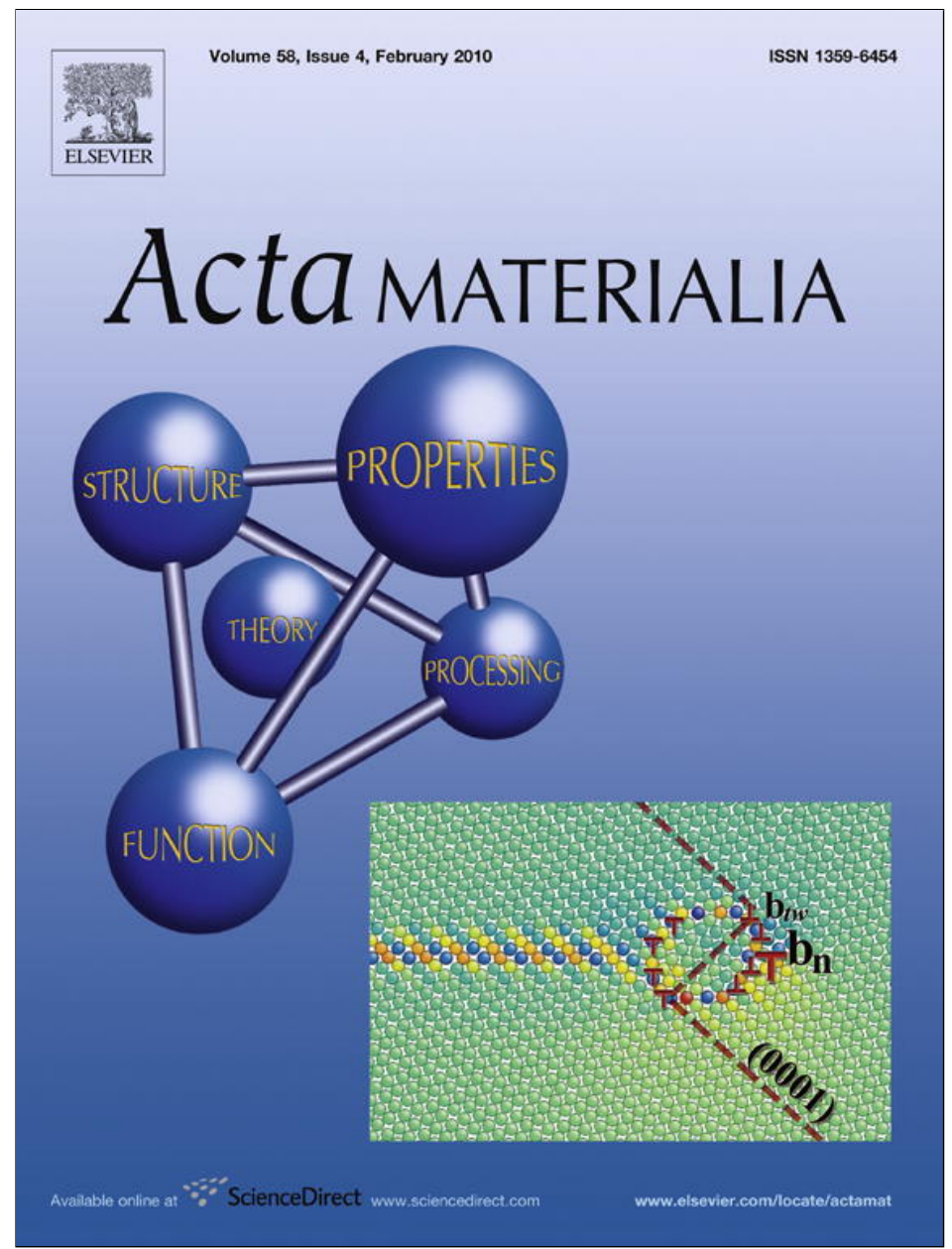

This article appeared in a journal published by Elsevier. The attached copy is furnished to the author for internal non-commercial research and education use, including for instruction at the authors institution and sharing with colleagues.

Other uses, including reproduction and distribution, or selling or licensing copies, or posting to personal, institutional or third party websites are prohibited.

In most cases authors are permitted to post their version of the article (e.g. in Word or Tex form) to their personal website or institutional repository. Authors requiring further information regarding Elsevier's archiving and manuscript policies are encouraged to visit:

http://www.elsevier.com/copyright 


\title{
Atomistic modeling of interfaces and their impact on microstructure and properties
}

\author{
Y. Mishin ${ }^{\mathrm{a}, *}, \mathrm{M}$. Asta $^{\mathrm{b}}, \mathrm{Ju} \mathrm{Li}^{\mathrm{c}}$ \\ ${ }^{a}$ Department of Physics and Astronomy, MSN 3F3, George Mason University, 4400 University Drive, Fairfax, VA 22030, USA \\ ${ }^{\mathrm{b}}$ Department of Chemical Engineering and Materials Science, University of California Davis, One Shields Ave., Davis, CA 95616-5294, USA \\ ${ }^{\mathrm{c}}$ Department of Materials Science and Engineering, University of Pennsylvania, 3231 Walnut Street, Philadelphia, PA 19104, USA
}

Received 14 February 2009; received in revised form 14 August 2009; accepted 24 October 2009

Available online 1 December 2009

\begin{abstract}
Atomic-level modeling of materials provides fundamental insights into phase stability, structure and properties of crystalline defects, and to physical mechanisms of many processes ranging from atomic diffusion to interface migration. This knowledge often serves as a guide for the development of mesoscopic and macroscopic continuum models, with input parameters provided by atomistic models. This paper gives an overview of the most recent developments in the area of atomistic modeling with emphasis on interfaces and their impact on microstructure and properties of materials. Modern computer simulation methodologies are discussed and illustrated by several applications related to thermodynamic, kinetic and mechanical properties of materials. Existing challenges and future research directions in this field are outlined.
\end{abstract}

(c) 2009 Acta Materialia Inc. Published by Elsevier Ltd. All rights reserved.

Keywords: Atomistic modeling; Interatomic potentials; First-principles methods; Molecular dynamics; Interfaces

\section{Introduction}

Over the past two decades, computer simulations based on atomistic models have become a powerful tool for gaining fundamental knowledge of materials processes and properties, and for providing input data for continuum models and materials design [1,2]. The capabilities and areas of application of such simulations continue to grow, reinforced by the progress in modern computer technology and the development of new simulation methods and numerical algorithms. The goal of this paper is to review recent advances in atomic-level materials modeling with emphasis on interfaces and their impact on microstructure and properties.

Any atomistic simulation starts with establishing a model describing atomic interactions in the material of

\footnotetext{
* Corresponding author. Tel.: +1 703993 3984; fax: +1 7039931993.

E-mail address: ymishin@gmu.edu (Y. Mishin).
}

interest. The two main approaches currently used to this end are: (i) first-principles ( $\mathrm{ab}$ initio) methods based on a quantum-mechanical treatment of electrons and (ii) classical interatomic potentials. The advantage of first-principles calculations, in addition to being accurate and having strong physical underpinnings, is the ability to treat both pure elements and multicomponent systems with a comparable amount of computational effort. This advantage greatly facilitates the exploration of chemical effects on materials properties. At the same time, the numerical complexity and $N^{3}$ scaling of first-principles methods make them computationally demanding. With today's computers, the largest systems that can be relaxed statically contain a few hundred atoms. Ab initio molecular dynamics (MD) can be run for times on the order of tens of picoseconds; to put this number in perspective, a typical period of atomic vibrations in solids is $\sim 0.1 \mathrm{ps}$. Despite these limitations, even calculations within this small domain of sizes and times often provide invaluable insights into the physics of many materials processes. 


\section{List of abbreviations}

ACI amorphous-crystal interface

ADP angular-dependent potential

BOP bond-order potential

CALPHAD calculation of phase diagrams

CE cluster expansion

CPA coherent-potential approximation

CSL coincident site lattice

DDD discrete dislocations dynamics

DFT density functional theory

DFPT density functional perturbation theory

EAM embedded-atom method

FE-NEB free-end nudged elastic band

FS Finnis-Sinclair

GB grain boundary
GGA generalized gradient approximation

GSF generalized stacking fault

HF Hartree-Fock

HRTEM high-resolution transmission electron microscopy

KMC kinetic Monte Carlo

LDA local density approximation

MC Monte Carlo

MEAM modified embedded-atom method

MD molecular dynamics

NEB nudged elastic band

TEM transmission electron microscopy

TST transition state theory

VASP Vienna Ab Initio Simulation Package
There are other processes and properties, however, that are presently inaccessible by first-principles methods and are likely to remain so for years to come. For example, some aspects of plastic deformation and fracture, multicomponent diffusion, melting, crystallization and other phase transformations (including phase nucleation and growth) involve large ensembles of atoms and/or require statistical averaging over many atomic events. The intrinsic length and time scales of these processes are simply beyond the spatiotemporal domain currently accessible by firstprinciples methods. Computer modeling of such processes is made possible by the use of semi-empirical interatomic potentials. Potential-based methods permit fast calculations of the system energy and classical interatomic forces, and give access to systems containing millions of atoms. They enable MD simulations to run for tens or even hundreds of nanoseconds, and allow Monte Carlo (MC) runs for millions of trial moves per atom. Even these capabilities often prove to be too restrictive, and new approaches seeking to further expand the length and time scales of the regular MD and MC methods are being actively pursued [3-9].

This paper has the following structure. After reviewing the first-principles methods (Section 2), we discuss in detail the current status in the development of interatomic potentials (Section 3). The latter topic is especially important because many simulation results reported in the literature are, in effect, as reliable as the potentials are. Section 4 gives a brief overview of the most common atomistic simulation methods and computer codes. The rest of the paper is devoted to examples of recent applications of atomistic methods, ranging from interface structure and thermodynamics to mechanical properties and interface migration. Clearly it is impossible to cover all applications in one article. Although our selection of the topics was admittedly influenced by our own interests and expertise, our main goal was to highlight several areas in which significant pro- gress has recently been achieved and where further active developments can be expected in the near future. Given that the selected applications are centered around interfaces, microstructure and mechanical behavior, it is not surprising that a certain preference is given to potentialbased simulations vs. first-principles calculations. Whenever possible, we discuss how atomistic information can be passed to larger-scale simulations methods, such as phase-field modeling [10] and finite-element calculations [11]. These continuum approaches are reviewed in other papers of this cluster $[10,11]$.

Because of the space limitation, our discussion will be focused on work published over the past approximately 15 years. The interested reader is referred to earlier literature, especially to the Sutton and Balluffi book [12], for a broad overview of the developments before 1995. Furthermore, we had to focus the discussion on metallic materials, and regard ceramics, semiconductors, polymers and other important classes of materials as lying beyond the scope of the paper. We conclude the paper (Section 10) by pointing to a few research directions where future breakthroughs are likely to occur due to developments of new methods or extension to new applications.

\section{First-principles calculations}

\subsection{Methods, computer codes and perspectives}

First-principles calculations (in the context of atomistic modeling) solve the electronic-structure problem in order to obtain the Born-Oppenheimer energy surface $U\left(\mathbf{r}_{1}\right.$, $\left.\mathbf{r}_{2}, \ldots, \mathbf{r}_{N}\right)$, where $\left\{\mathbf{r}_{1}, \mathbf{r}_{2}, \ldots, \mathbf{r}_{N}\right\}$ are the nuclear positions [2]. The Born-Oppenheimer approximation decouples the electronic degrees of freedom from the nuclear degrees of freedom, due to a large mass ratio between the nuclei and electrons. The electron problem is inherently quantum-mechanical. $U\left(\mathbf{r}_{1}, \mathbf{r}_{2}, \ldots, \mathbf{r}_{N}\right)$ includes the electron 
kinetic energy, electron-electron, electron-nucleus and nucleus-nucleus potential energies, but not the nuclear kinetic energy. Also, most ab initio calculations employing the basic density functional theory (DFT) $[13,14]$ assume the electronic configuration to be in an instantaneous ground state for each nuclear configuration. It is also possible to investigate excited electronic states and their effects on nuclei dynamics, using first-principles methods such as the time-dependent DFT [15]. Such problems can arise in materials science in the interaction of light with matter and transport, e.g. in exciton and polaron dynamics [16].

If $U\left(\mathbf{r}_{1}, \mathbf{r}_{2}, \ldots, \mathbf{r}_{N}\right)$ is computed on-the-fly to drive Newtonian dynamics for the nuclei, it is called first-principles MD [17-19]. For light-mass nuclei such as hydrogen, sometimes even the nucleus dynamics need to be treated quantum-mechanically $[20,21]$. None of the first-principles methods solve the electron/nuclei problem exactly: they are all based on various layers of approximations. However, these approximations have rigorous quantitative foundations and are at least nominally improvable systematically. The accuracy of the approximations is often quite good, and sometimes excellent, within its range of validity and computability for practical applications. The philosophy of ab initio calculations is striving to make quantitative predictions using only the Planck constant, electron mass, nuclear charge and other universal constants at a quantum-mechanical level, with an expressed goal of approaching chemical accuracy.

For extended systems, especially metals, the KohnSham DFT [14] is the tool of choice. Their procedure maps a many-interacting-electrons system, with a many-body wavefunction $\Psi\left(\mathbf{r}_{1}, \mathbf{r}_{2}, \ldots, \mathbf{r}_{M}\right)$, to a unique non-interacting-electrons system $\left\{\psi_{1}(\mathbf{r}), \psi_{2}(\mathbf{r}), \ldots, \psi_{M}(\mathbf{r})\right\}$ with the same electron density, where the $\psi_{i}(\mathbf{r})$ s are single-electron wavefunctions and $M$ is the number of electrons. The beauty of this mapping is that the kinetic energy of the reference non-interacting-electrons system largely captures the nonlocal component of $U$. Also, the availability of $\left\{\psi_{1}(\mathbf{r}), \psi_{2}(\mathbf{r}), \ldots, \psi_{M}(\mathbf{r})\right\}$ allows one to define the so-called exchange interaction in reference to the well-known Hartree-Fock (HF) formalism [22], and the residual is defined as the correlation energy. Thus, by definition, a HF calculation has $100 \%$ exchange energy but $0 \%$ correlation energy.

The key ingredients of DFT calculations are (i) density functionals, (ii) basis functions and (iii) treatment of core electrons. The best-known density functionals include the local density approximation (LDA) [23-25], the PW91 generalized gradient approximation (GGA) [26,27], the PBE GGA $[28,29]$ and hybrid functionals, such as B3LYP [30], in which the exact HF exchange energy is mixed with LDA/GGA approximants. Developing physically based, accurate and easy-to-compute electron density functionals is a very active field of research in the physics and chemistry communities [31].

For the basis functions, the planewave basis has an algorithmic simplicity and continuously tunable precision, and therefore is a very popular choice adopted in the Vienna $\mathrm{Ab}$ Initio Simulation Package (VASP) [32,33], ABINIT [34], PWscf [35], Dacapo [36], Quantumespresso [37] and other packages. Augmented planewave codes such as WIEN2k [38] and the muffin-tin orbital basis are also quite popular. The Gaussian basis is used in quantum chemistry packages such as GAUSSIAN [39], GAMESS [40], NWChem [41] and Q-Chem [42], as well as entirely DFT packages such as SIESTA [43] and FIREBALL [44]. Finally, the so-called real-space DFT methods based on finite differences or finite elements are also under active development [45-47].

In the so-called all-electron DFT calculations, all electrons are treated in the same framework, which obviously makes the calculations computationally demanding. Since the core electrons of atoms often do not participate in bonding, pseudopotentials are constructed to mimic the effect of core electrons on valence electrons. The pseudopotentials are typically linearly additive, nonlocal quantum-mechanical operators, fitted to isolated-atom full-electron wavefunctions. These pseudopotentials for electrons surrounding a collection of "pseudoatoms" tend to be much more transferable than empirical interatomic potentials are. The main idea behind a nonlocal pseudopotential is a mapping of the actual valence electron wavefunction $\psi(\mathbf{r})$ to a pseudowavefunction $\tilde{\psi}(\mathbf{r})$, where $\tilde{\psi}(\mathbf{r})$ is smoother and requires fewer planewaves to represent. The so-called norm-conserving pseudopotentials [48] preserve the net electron charge inside and outside an artificial radial cutoff. These are quite accurate, but can require a large number of planewaves to converge for many elements (e.g. carbon, oxygen, $3 d$ metals and $f$-electron systems). The so-called ultrasoft pseudopotentials $[49,50]$ and the projector augmented-wave method [51] drastically improve computational efficiency without sacrificing much accuracy and transferability for these elements.

First-order derivatives of $U\left(\mathbf{r}_{1}, \mathbf{r}_{2}, \ldots, \mathbf{r}_{N}\right)$, such as forces and the stress tensor, can be calculated analytically [52] by taking the inner product of certain operators between the present wavefunctions $\left\{\psi_{1}(\mathbf{r}), \psi_{2}(\mathbf{r}), \ldots, \psi_{M}(\mathbf{r})\right\}$ only. These are in fact special cases of the general linear response theory (density functional perturbation theory, DFPT), in which one imagines an arbitrary perturbation in the DFT calculation and analytically derives closed-form expressions for the response. DFPT has been used to calculate phonon frequencies with great success [53]. Lattice constants, stresses (which lead to elastic moduli) and phonon frequencies are important quantities since they can be compared with experimental results and can serve to validate the accuracy of ab initio methodologies [54-56].

\subsection{Application to compositionally disordered materials}

First-principles calculations for compositionally disordered materials are commonly performed within the framework of the cluster-expansion (CE) formalism [57-66]. In 
this approach, the energy for a given configuration of atomic species on an underlying parent lattice (e.g. facecentered cubic (fcc), body-centered cubic (bcc) or hexagonal close-packed (hcp)) is parameterized in terms of pair and multibody cluster interactions. These cluster interactions are computed either within the framework of structure-inversion methods $[58,60,62,66]$, involving fitting the $\mathrm{CE}$ to energies of periodic ordered structures, or by techniques based on the coherent-potential approximation (CPA) theory for the electronic structure of a random alloy [64,67-70]. Once a converged CE has been derived, it can be coupled with direct enumeration techniques to determine the energetically preferred atomic arrangements on the given lattice $[59,60,63]$. Further, by employing CEs in MC simulations or cluster-variation method calculations, enthalpies and entropies of mixing and phase boundaries on phase diagrams can be computed (see e.g. Ref. [59,63] and references therein).

For many alloy systems it has been found that, in order to obtain good agreement with experimental measurements, it is necessary to account for non-configurational contributions to mixing free energies, arising from electronic $[63,64,71]$ and vibrational excitations $[63,64,72,73]$. For solid solutions, in systems where short-range order can be neglected, computationally less demanding approaches for calculating the energetics of random alloys involve the use of the CPA $[67,68,70]$ or special quasi-random structures [74-78], which are ordered structures designed to have correlation functions approximating those of a disordered alloy.

Applications of the $\mathrm{CE}$ and related formalisms have been recently reviewed [59,63,64], and for many alloy systems impressive agreement with experimental measurements has been demonstrated. Active research continues in further development of such techniques to address a number of outstanding challenges. Such challenges include systems where long-range interactions arise due to size-mismatch-related elastic relaxations [79-81], where the electronic structure displays strong variations with atomic configurations [58,64], and where crystal structures are mechanically unstable over a range of compositions [78,82-84]. Extensions of the CE formalism are also being actively pursued for applications to inhomogeneous systems, including surfaces [85-88], to calculations of tensorial quantities $[65,89]$ and to the modeling of diffusion in nondilute systems $[90,91]$.

While the CE methods described above have been used extensively in calculations for binary and some ternary systems, their computational demands grow rapidly with increasing number of components, and they remain intractable in direct applications to commercial multicomponent alloys. Hence, in the context of alloy design, first-principles thermodynamic and kinetic methods are often combined with CALPHAD (calculation of phase diagrams) approaches $[92,93]$ to model complex multicomponent systems. In this approach, first-principles methods are employed to provide thermochemical and kinetic data, or to augment experimental information in the develop- ment of accurate thermodynamic and mobility databases. Here a primary challenge lies in estimating the error in the first-principles calculations, in order to give them proper weight in the construction of such databases [94]. Discussions of the strategies for coupling first-principles and CALPHAD methods, along with some illustrative examples, can be found in Refs. [63,94-98].

\section{Interatomic potentials}

\subsection{Why do we need interatomic potentials?}

Most atomistic simulations employ the MD and MC methods implementing various statistical ensembles (Section 4). These methods require multiple evaluations of the total potential energy of the system $U$ and, in the case of MD, the Newtonian forces $\mathbf{F}_{i}=-\partial U / \partial \mathbf{r}_{i}$ acting on individual atoms ( $\mathbf{r}_{i}$ being the vector giving the position of an atom $i$ ). As discussed in Section 2.1, the most accurate calculations of energies and forces are made by first-principles methods based on quantum-mechanical treatment of electrons. However, there are many physical processes whose natural length and time scales lie way outside the domain currently accessible by first-principles methods. Access to them is afforded by semi-empirical interatomic potentials [99].

Interatomic potentials parameterize the configuration space of the material and express $U$ as a relatively simple function of all atomic positions (configuration point). The forces are then computed (usually, analytically) as coordinate derivatives of $U$. This computation of $U$ and $\mathbf{F}_{i}$ is a simple and very fast numerical procedure with an order- $N$ scaling. It does not involve any quantum-mechanical calculations, although they are often used during the development of potentials. The potential functions contain fitting parameters, which are adjusted to reproduce selected properties of the material known from experiment and/or first-principles calculations. Once the fitting process is complete, the parameters are fixed once and for all and the potential is used in all simulations of the given material. The underlying assumption is that a potential which gives accurate energies/forces on configuration points used during the fit will also give reasonable results for configurations between and beyond those points. This property of potentials is referred to as "transferability" and is the most adequate measure of their quality.

Clearly, interatomic potentials can never be as accurate as first-principles calculations. However, computational speeds are limited, while many processes occur on large, and often multiple, length and time scales. It is often more useful to have an approximate answer for a process on its natural scale and at realistic temperatures than to rely on very accurate numbers for small models computed at $0 \mathrm{~K}$. Despite the increasing efficiency of first-principles methods, there will always be a need to push the length and time scales of simulations even further, or to extend them to more complex systems. 


\subsection{The embedded-atom method}

Empirical potentials have been used for materials modeling for several decades. Early work was based on simple pair potentials, such as the Lennard-Jones and Morse forms. Despite the oversimplification of atomic bonding, pair potentials have been very useful for producing valuable insights into materials behavior on the atomic level. Many of the early results obtained with pair potentials have proved to be fundamentally correct despite later refinements of the numerical values. Pair potentials continue to be employed today for fundamental studies of generic properties of materials. However, they are hardly capable of making quantitative predictions and in many cases can be wrong even qualitatively.

Presently, the most widely used potential format for metallic systems is given by the Finnis-Sinclair (FS) method [100] and the embedded atom method (EAM) [101]. Although initially derived from different physical approaches (DFT [101,102] and second-moment tight binding $[100,103])$, the two potential forms are similar. The choice of the potential format is usually dictated by tradition (e.g. most American groups prefer EAM while many European groups favor FS) rather than accuracy or any other advantages. The capabilities and limitations, as well as the potential generation procedures, are common to both potentials and will be discussed here using EAM as an example.

In EAM, $U$ is postulated in the form

$U=\frac{1}{2} \sum_{i, j(j \neq i)} \Phi_{s_{i} s_{j}}\left(r_{i j}\right)+\sum_{i} F_{s_{i}}\left(\bar{\rho}_{i}\right)$,

where the first term is the sum of pair interactions, $\Phi_{s_{i} s_{j}}\left(r_{i j}\right)$ is the pair-interaction potential between atom $i$ (of chemical identity $s_{i}$ ) and atom $j$ (of chemical identity $s_{j}$ ), and $F_{s_{i}}$ represents the embedding energy of atom $i$ in the host electron density $\bar{\rho}_{i}$ induced at site $i$ by all other atoms. The host electron density is given by [101]

$\bar{\rho}_{i}=\sum_{j \neq i} \rho_{s_{j}}\left(r_{i j}\right)$

where $\rho_{s_{j}}(r)$ is the electron density function assigned to atom $j$. For an FS potential [100], $\bar{\rho}_{i}$ is given by a sum of additional pairwise functions similar to the first term in Eq. (1). The second term in Eq. (1) represents, in an approximate manner, the many-body interactions responsible for a significant part of bonding in metals. The form of the embedding function is arbitrary in EAM but is postulated to be the negative of the square root of $\bar{\rho}_{i}$ in the FS method.

An EAM description of an elemental metal is given by three functions: $\Phi(r), \rho(r)$ and $F(\bar{\rho})$. A binary system A$\mathrm{B}$ is represented by seven functions $\Phi_{A A}(r), \Phi_{A B}(r), \Phi_{B B}(r)$, $\rho_{A}(r), \rho_{B}(r), F_{A}(\bar{\rho})$ and $F_{B}(\bar{\rho})$. If potential functions for two metals A and B are available, only the cross-interaction potential $\Phi_{A B}(r)$ is needed for a full description of the binary system. The advantage of this approach is that each element is represented by the same set of functions both in its pure state and in all alloys and compounds involving that element. A complete EAM description of an $n$-component system requires $n(n+1) / 2$ pair interaction functions $\Phi_{s s^{\prime}}(r), n$ electron density functions $\rho_{s}(r)$ and $n$ embedding functions $F_{s}(\bar{\rho})(s=1, \ldots, n)$. All these functions can be subject to certain mathematical transformations (called invariant transformations) that do not change the energy or forces calculated with the potential $[99,102]$. (The fixed square-root form of the embedding function and the form of the host electron density in the FS method preclude most of the invariant transformations.) The pair-interaction and electron-density functions together with several derivatives are truncated to zero at a cutoff distance $r_{c}$ covering 3-5 coordination shells. EAM functions are traditionally defined by analytical expressions with several adjustable parameters, although some authors prefer cubic splines or a set of basis functions.

In the EAM formalism, it is straightforward to derive analytical expressions for interatomic forces, elastic constants, the dynamical matrix, the mechanical stress tensor, unrelaxed vacancy formation energy, surface energies for different crystal orientations and a number of other properties [99]. Because such expressions include only the potential functions and their first and second derivatives, they can be easily coded into simulation programs and used for fast calculations of these properties.

EAM and FS potentials currently serve as the main workhorse for atomistic simulations of metallic materials. They have been applied to simulations of interfaces, dislocations, fracture, diffusion, structural transformations, solidification and melting, and many other processes. Over the past two decades, these potential forms have produced an excellent record of delivering reasonable values of elastic moduli, phonon dispersion relations, thermal expansion, melting properties, stacking fault energies, vacancy formation and migration energies, surface energies, surface relaxations and reconstructions, and many other properties of metals. For binary systems, experimental heats of formation and other properties of ordered compounds can be reproduced with reasonable accuracy. For some binary systems, even basic features of the phase diagrams can be reproduced without fitting to experimental thermodynamic data $[104,105]$ (see the example in Fig. 1).

EAM and FS potentials are available for a large number of elemental metals as well as binary systems. Some of them have been successfully tested for a wide range of properties and are widely used, such as the potentials for Al [106-108], $\mathrm{Ni}$ [106,108,109], $\mathrm{Cu}$ [109,110], Au [109], Fe [111] and Ni-Al $[104,112]$. A useful repository of potentials can be found at the NIST website, http://www.ctcms.nist.gov/potentials/. Some potentials accurately reproduce only specific properties of the material but perform poorly on other properties, making them useful for only certain types of applications. Several potentials have been proposed for ternary systems (see e.g. [113-115]). Unfortunately, the quality of ternary 

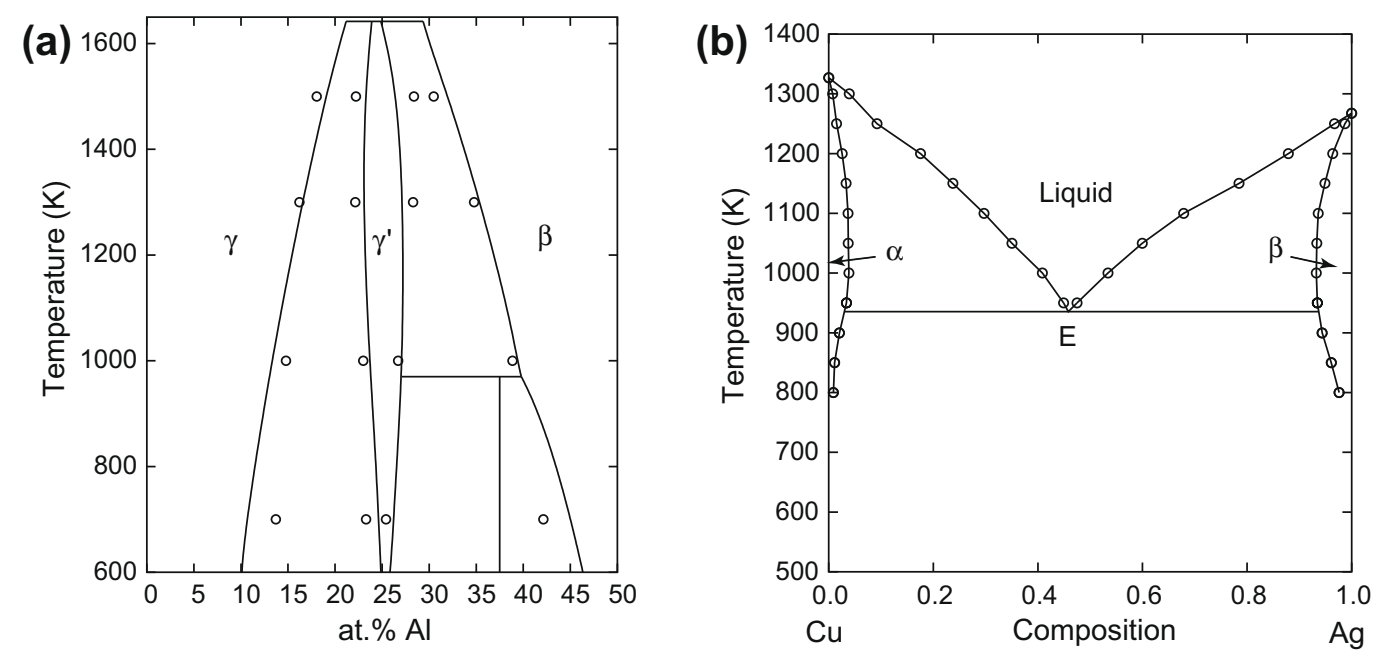

Fig. 1. Phase diagrams of the (a) Ni-Al [104] and (b) $\mathrm{Cu}-\mathrm{Ag}$ [105] systems calculated with EAM potentials (points). In (a) the lines show the experimental diagram; in (b) the lines simply connect the calculated points and serve as a guide to the eye.

potentials remains far from the level currently achieved for pure metals and binary alloys.

One of the challenges in EAM and FS simulations is the lack of flexibility in addressing chemical effects. Each time a new solute $B$ should be added to a metal A to examine its effect on a particular property, a new binary potential A-B must be constructed (unless it already exists). The construction of an accurate binary potential is a highly demanding task. This explains why a common strategy in this field is to generate new potentials that are not only accurate but also "universal", i.e. applicable to a wide range of simulations reaching beyond the particular problem that motivated the effort. Although ad hoc potentials applicable to only one particular type of simulations abound in the literature, they make little impact on the field.

\subsection{Potential generation procedures}

The choice of the fitting database is a critical step that affects the accuracy and reliability of new potentials. While early potentials were fit to a few experimental numbers, the current trend is to include both experimental information and a large amount of first-principles data $[104,105,107,108,110,112,116-120]$. In fact, some of the recent potentials have been fit predominantly to first-principles data, essentially making them a parameterization of first-principles calculations. The incorporation of firstprinciples information can drastically improve the reliability of potentials by sampling larger areas of configuration space and including configurations far away from regions represented by experimental data. Typical experimental properties used for potential fitting include the cohesive energy $E_{0}$, lattice parameter $a_{0}$, elastic constants $c_{i j}$, vacancy formation energy and often the stacking fault energy.

First-principles information is usually included in the form of energy-volume functions (binding relations) for the ground-state structure and several hypothetical alternate structures of the same metal. For binary systems, binding relations for a number of ordered compounds with different crystal structures and stoichiometries can be included [104,105,112,116,118,121], often covering the full composition range. Such compounds may or may not exist on the experimental phase diagrams. Their only role is to sample different local environments and atomic volumes of the material, including highly non-equilibrium configurations that may occur during atomistic simulations.

In addition to crystal structures, first-principles energies along homogeneous deformation paths are sometimes included, such as the tetragonal deformation path between fcc and bcc structures (the Bain path) or the trigonal deformation path fcc-simple-cubic-bcc (Fig. 2). Another example is the affine shear deformation along a twinning path returning, for example, the fcc structure back to itself but with a twin orientation. An alternative approach is to fit potentials to first-principles forces drawn from snapshots of $\mathrm{ab}$ initio MD simulations for solid and liquid phases (the force-matching method) [107].

The fitting parameters of potentials are optimized by minimizing the weighted mean-squared deviation of properties from their target values. The weights offer a powerful tool for controlling the priority of some properties over others. In recent years, optimization algorithms have been proposed that seek to avoid overfitting or underfitting of the database $[108,122]$. Rigorous statistical methods have been applied [123], and even approaches based on neural networks [124,125] and genetic algorithms $[126,127]$ have been proposed. A portable computer software has been developed [128] to improve the efficiency of the fitting process. Nevertheless, the construction of highquality potentials remains something of an art [129]. Indeed, many of the target properties can be only determined by actual simulations (e.g. temperatures of phase transformations) and can hardly be included in the fit 
(a)

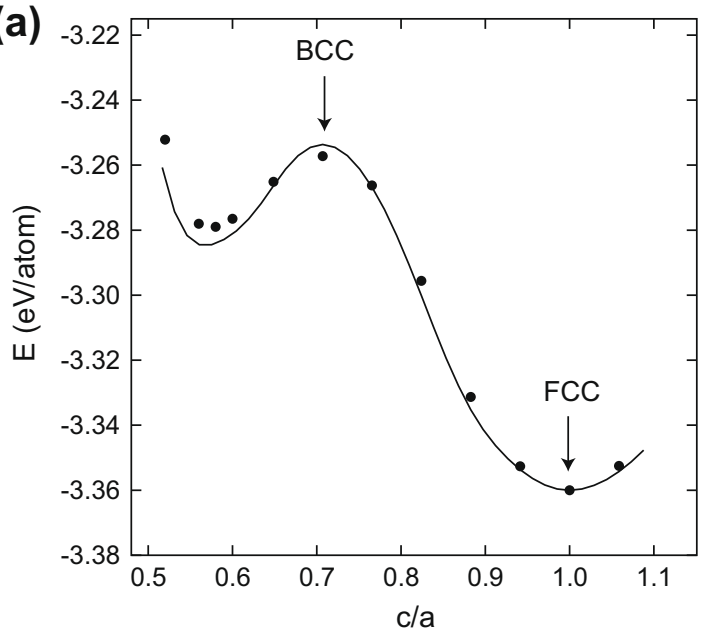

(b)

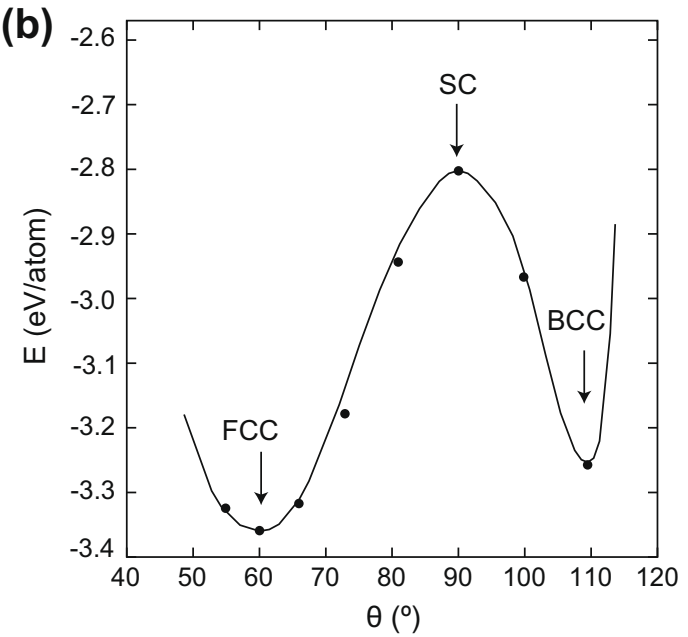

Fig. 2. Energy along (a) the Bain path and (b) the trigonal deformation path of Al obtained by first-principles calculations (points) and predicted by an EAM potential (curves) [108]. The arrows indicate the $c / a$ ratios and trigonal angles corresponding to the fcc, bcc and simple cubic structures. The volume is fixed at the equilibrium fcc value.

directly. Many properties can be "fitted" only indirectly, i.e. by making smart choices of representative atomic configurations, taking advantage of known correlations between different physical properties, or even relying on (often mysterious) correlations between certain features of potential functions (wiggles, humps and bumps) and particular physical properties.

Efficient fitting strategies for ternary or higher-order potentials have not been developed. For a ternary system, a simultaneous fit of all 12 functions of an EAM potential would probably be impractical - not to mention higherorder systems. A more promising approach could be to create a set of independent binary potentials $(\mathrm{A}-\mathrm{B}, \mathrm{B}-\mathrm{C}, \mathrm{A}-\mathrm{C}$, etc.), which together describe all interactions in the multicomponent system $\mathrm{A}-\mathrm{B}-\mathrm{C}$, etc. There is a risk, however, that properties of multicomponent compounds may not come out reasonable without a direct fit. In addition, if the invariant transformation parameters of an EAM potential have been optimized for two binaries, they cannot be used for fitting a third one; thus, the quality of the obtained multicomponent potential might depend on the order in which the constituent binaries are created. In principle, the invariant transformation parameters of all binaries can be optimized simultaneously, but this approach has not been explored.

\subsection{Other types of potentials}

EAM and FS potentials work best for simple and noble metals and are less accurate for transition metals, reflecting one of their intrinsic limitations (these are central-force models that cannot capture the covalent component of bonding due to $d$-electrons). Hcp metals also present a challenge to EAM. Several attempts have been made to address these limitations by generalizing the functional forms of the EAM and FS methods.
In the modified EAM (MEAM) [130-133], the electron density assigned to atoms is treated as a tensor and the host electron density $\bar{\rho}_{i}$ is expressed as a function of tensor invariants $\bar{\rho}_{i}^{(k)}(k=0,1,2,3)$ of several orders. Based on symmetry considerations, these invariants may be interpreted as representing the $s, p, d$ and $f$ electronic orbitals, although there is no direct link to the actual electronic structure of the material. MEAM also introduces a many-body "screening" procedure and limits all interactions to one or two coordination shells. Computationally, MEAM is slower than regular EAM but can be more accurate for transition metals. MEAM potentials have been constructed for a number of fcc [134-136], hcp [137,138] and bcc $[132,139]$ metals, as well as a few binary systems [140-144]. Even potentials for strongly covalent elements such as C [145], Si [130,131] and Ge [131] have been proposed, along with potentials for metal-nonmetal systems such as Fe-C [146], Fe-H [147], Ti-C and Ti-N [148].

The recently proposed angular-dependent potential (ADP) method $[118,119,149]$ introduces non-central interactions by postulating the energy in the form

$$
\begin{aligned}
U= & \frac{1}{2} \sum_{i, j(j \neq i)} \Phi_{s_{i} s_{j}}\left(r_{i j}\right)+\sum_{i} F_{s_{i}}\left(\bar{\rho}_{i}\right)+\frac{1}{2} \sum_{i, \alpha}\left(\mu_{i}^{\alpha}\right)^{2}+\frac{1}{2} \\
& \times \sum_{i, \alpha, \beta}\left(\lambda_{i}^{\alpha \beta}\right)^{2}-\frac{1}{6} \sum_{i} v_{i}^{2},
\end{aligned}
$$

where the Greek superscripts refer to Cartesian directions. The first two terms constitute the regular EAM format. The non-central character of bonding is captured by the three additional terms, which are invariants of the dipole vectors

$\mu_{i}^{\alpha}=\sum_{j \neq i} u_{s_{i} s_{j}}\left(r_{i j}\right) r_{i j}^{\alpha}$

and quadrupole tensors 
$\lambda_{i}^{\alpha \beta}=\sum_{j \neq i} w_{s_{i} s_{j}}\left(r_{i j}\right) r_{i j}^{\alpha} r_{i j}^{\beta}$,

$v_{i}$ being the trace of $\lambda_{i}^{\alpha \beta}$ :

$v_{i}=\sum_{\alpha} \lambda_{i}^{\alpha \alpha}$.

These expressions introduce two additional pairwise functions, $u_{s s^{\prime}}(r)$ and $w_{s s^{\prime}}(r)$, and with them some additional fitting flexibility. The additional terms depend on angles between interatomic bonds and directly penalize the total energy for deviations of atomic environments from cubic symmetry. They contribute to $U$ when a cubic crystal is subject to non-hydrostatic strains, thus affecting the elastic constants. They also contribute to formation energies of ordered compounds with such common structures as $\mathrm{L1}_{0}, \mathrm{~L}_{1}$ and $\mathrm{L1}_{2}$. The angular terms tend to increase the energy of lattice defects (areas of broken symmetry), leading to higher surface energies and more accurate vacancy migration barriers. The angular-dependent corrections can be especially important in chemically disordered alloys, amorphous solids and liquids.

The ADP method is based on essentially the same physical assumptions as MEAM but is somewhat simpler and computationally more efficient. While MEAM introduces the same tensor invariants through the electron density, ADP adds them directly to $U$. In addition, the many-body screening procedure of MEAM is replaced by a smooth cutoff including 4-6 coordination shells. The ADP method is similar to but more general than the embedded-defect method proposed by Pasianot et al. [150]. The recently developed Au potential [151] is based on a similar angular-dependent format. ADP potentials have been constructed for the $\mathrm{Fe}-\mathrm{Ni}$ [118] and $\mathrm{Cu}-\mathrm{Ta}$ [149] systems.

Among other proposed improvements of EAM, Webb and Grest [152] introduced a gradient correction to the host electron density, $\beta\left|\nabla \bar{\rho}_{i}\right|^{2}$ ( $\beta$ being an adjustable parameter), and were able to improve the agreement with experiment for liquid/vapor interface free energies of fcc metals. More recently, Wu et al. [153] have demonstrated an improvement in transferability by refitting the existing Voter and Chen EAM Al potential [106] with a gradient correction. Some progress has been achieved in incorporating the magnetic energy in semi-empirical potentials for iron [154-156].

A number of potentials have been developed for strongly covalent and ionic solids [129,157-161], but are not discussed here. Many of them rely on sophisticated functional forms intended to capture environment-dependent interactions, bond breaking/forming terms, bondangle penalties and other effects. While probably not directly applicable to metallic systems, they could inspire new ideas for improved metallic potentials. In fact, Mueller et al. [162] have recently constructed an analytical bondorder potential (BOP) for $\mathrm{Fe}$ and were able to reproduce a variety of bulk, defect and surface properties of both the bcc and fcc phases, as well as the fcc-bcc transition temperature. Further comments on BOPs and their applications can be found in Section 10 .

\section{Methods of atomistic simulations}

Atomistic simulation is the offspring of the atomic worldview of Democritus-Boltzmann married to modern computing technology. It uses discrete nuclear positions $\mathbf{r}^{3 N} \equiv\left\{\mathbf{r}_{1}, \mathbf{r}_{2}, \ldots, \mathbf{r}_{N}\right\}$ as degrees of freedom to represent behavior of materials. Typically a potential energy function $U\left(\mathbf{r}^{3 N}\right)$ is assigned (see Sections 2 and 3 ) that depends on the chemical types of the atoms. Then some dynamics (in the broad sense) is run in the computer based on $U\left(\mathbf{r}^{3 N}\right)$, to generate new $\mathbf{r}^{3 N}(t)$ labeled by $t$, which can be a continuous time or an integer progress variable. The $\mathbf{r}^{3 N}(t)$ samples or trajectories with associated energies and forces are then processed to represent some characteristic structural, energetic or dynamical features of a material.

The simplest dynamics to follow is that of Newton: $m_{i} \ddot{\mathbf{r}}_{i}=-\partial U / \partial \mathbf{r}_{i}$, in which case we run MD computer simulations [163-165]. However, other "fictitious dynamics", such as viscous steepest descent $\left(\dot{\mathbf{r}}_{i}=-v \partial U / \partial \mathbf{r}_{i}\right)$ or conjugate-gradient energy minimization, or stochastic dynamics, such as Langevin dynamics or MC, can also be simulated, driven by the same $U\left(\mathbf{r}^{3 N}\right)$ [166-170]. The point of doing "fictitious" dynamics rather than "real" dynamics in the computer is that they can be computationally more efficient for investigations of certain aspects of a physical problem. Because atomistic simulations share the same discrete-atom worldview as classical chemical kinetics and statistical mechanics, simulation methods are often deeply rooted in analytical formulations developed in those fields. An effective simulation is often due to a conceptual insight, as well as algorithmic advances. For instance, the concept of inherent structures [171] in liquids and their mapping [172,173] have greatly facilitated the understanding of supercooled liquids and the glass transition. In solids, vibrational normal mode analysis of atoms can be used to characterize structural stability [169] and estimate free energy $[174,175]$. Lattice dynamics calculations of phonon spectra [176] and the vibrational density of states [177] can provide much needed insight beyond plain MD trajectories, and can even correct for certain quantum effects $[178,179]$.

Like MD, MC simulations offer a way to sample the energy landscape. Two important developments in MC approaches have been the umbrella sampling $[168,180$ 183], which improves the sampling efficiency, and the Wang-Landau algorithm for computing the density of states [184]. The atoms can be on-lattice (different chemical types or vacancies/interstitials move on a lattice) or off-lattice, and can also be added or extracted from the simulation (grand canonical MC). Instead of sampling equilibrium fluctuations, kinetic Monte Carlo (KMC) simulations [185-188] sample saddle-point transitions on the potential energy landscape according to transition-state theory (TST) [189] in order to model rate phenomena in non-equilibrium systems. The KMC algorithm can also be applied to model the dynamics of discrete defects such as dislocation segments [190] or shear transformation zones [191] at the mesoscale. 
The time scale accessible to direct MD simulations is constrained by the necessity to temporally resolve the highest-frequency vibrations in the atomic system. On the other hand, according to the TST expression $R=v \exp \left(-Q / k_{\mathrm{B}} T\right)$, the success rate $R$ of a thermally activated event can be much smaller than the physical attempt frequency $v$, if the activation energy $Q \gg k_{\mathrm{B}} T[192,193]\left(k_{B}\right.$ is the Boltzmann factor). Therefore, for such rare-event problems, a direct MD simulation is not the best approach. To overcome the time-scale limitation, one can compute $Q$ and $v$ directly using saddle-point search methods such as the nudged elastic band (NEB) [194-198] or the Dimer [199] methods. For stress-driven events where the final free-energy minimum may be much further away from the saddle than the initial minimum, a modification to the NEB method called the free-end NEB was developed [200,201], which allows for more efficient sampling of the minimum energy path around the saddle. Other methods to accelerate simulations of sequential rare events [192] include activation-relaxation [202], hyperdynamics [4], the bond-boost method [203,204], metadynamics [205,206], parallel replica dynamics [5] and temperature-accelerated dynamics [6]. All these methods aim to improve the efficiency of sampling rare events while preserving the statistical characteristics in traversing the energy landscape.

Despite the conceptual and algorithmic advances in atomistic simulations mentioned above, the large-scale "brute-force" MD simulation is still needed in many cases, especially in situations involving transient dynamics (such as shocks) and complex phase changes/geometries and loading conditions [207-211]. Software such as LAMMPS [212], IMD [213] and DL_POLY [214] are publicly available to perform large-scale MD simulations on parallel platforms.

\section{Equilibrium properties of interfaces}

\subsection{Grain boundary structure}

Grain boundaries (GBs), i.e. interfaces between differently oriented crystals of the same material, are critical for many properties of materials [12]. The computer modeling of GBs has been mostly focused on symmetrical boundaries, i.e. boundaries which possess mirror symmetry of crystallographic planes and directions across the boundary plane [12,215]. Asymmetrical boundaries have also been studied [12,216] but not as extensively as symmetrical ones. It is known, however, that most GBs in real polycrystalline materials are asymmetrical [217-219]. In fact, they are often curved and sample a range of different asymmetrical planes. Unfortunately, the current understanding of atomic structure, energies $\gamma$ and mechanical properties of asymmetrical GBs remains rather limited. Recent experimental work [220-226] and atomistic simulations [227-235] have revealed a rich variety of their structures, structural transformations, dissoci- ation reactions, faceting transitions and other interesting properties. Our discussion will focus on asymmetrical boundaries as they are likely to attract much attention in the future as simulations extend to more realistic polycrystalline systems.

In a recent study [234], the energies and equilibrium atomic structures of asymmetrical $\Sigma 11$ [110] tilt GBs $\left(\theta=50.479^{\circ}\right.$ around the $[110]$ axis $)$ in $\mathrm{Cu}$ have been computed over the entire range of inclination angles $\alpha(\Sigma$ is the reciprocal density of coincident sites). The two well-known symmetrical boundaries of this family occur on the crystal planes $(3 \overline{3} \overline{2})\left(\alpha=0, \gamma=0.702 \mathrm{~J} / \mathrm{m}^{2}\right)$ and (1 $\left.\overline{1} 3\right)\left(\alpha=90^{\circ}\right.$, $\left.\gamma=0.310 \mathrm{~J} / \mathrm{m}^{2}\right)$, with asymmetrical boundaries at intermediate inclinations. By crystal symmetry, this $90^{\circ}$ angular range covers all distinct GB structures of this family.

It was found that these boundaries dissociate into a lowangle GB formed by Shockley partial dislocations and a high-angle non- $\Sigma 11$ boundary. (The latter boundary is not $\Sigma 11$ because the local lattice misorientation near this boundary deviates from the angle producing the $\Sigma 11$ coincident site lattice (CSL), resulting in an incommensurate (001)(111) plane-matching boundary [234].) The two product boundaries are separated by a layer of an fcc-based long-period structure containing an array of intrinsic stacking faults. This dissociation can be considered as a generalization of the 9R-phase formation found previously by simulations and high-resolution transmission electron microscopy (HRTEM) at incoherent $\Sigma 3$ twin boundaries in fcc metals [220,236-238]. This dissociation with the formation of long-period structures is likely to be a generic feature of all [1 10] tilt boundaries in fcc metals with low stacking-fault energies.

The simulations have also revealed that asymmetrical GBs tend to break into nanometer-scale facets. The facet planes often correspond to a nearby symmetrical boundary with a low energy (Fig. 3a), but not always. For example, the $\Sigma 11$ [1 10 ] tilt GBs form facets that are not $\Sigma 11$ and do not even belong to any particular CSL [234] (Fig. 3b).

The latter finding is important as it exposes a significant limitation of the CSL model for GBs. Indeed, the simulations [234] probed a trajectory in the five-dimensional space of geometric parameters on which four angles were fixed, imposing a $\Sigma 11 \mathrm{CSL}$, whereas the inclination angle was varied. The trajectory connected the two symmetrical boundaries and sampled a series of asymmetrical orientations between them. The traditional CSL model prescribes that the $\Sigma 11$ misorientation should be preserved at each point of the GB despite any changes in its local inclination. Contrary to this, it was found [234] that all asymmetrical GBs along this trajectory contained nanofacets of a GB external to this trajectory. This external GB is an incommensurate, non- $\Sigma$ boundary with a relatively low energy. Thus, the asymmetrical GBs locally deviate away from the macroscopically imposed CSL misorientation and incorporate facets of a non- $\Sigma$ boundary, which reduces the total GB energy. 
(a)

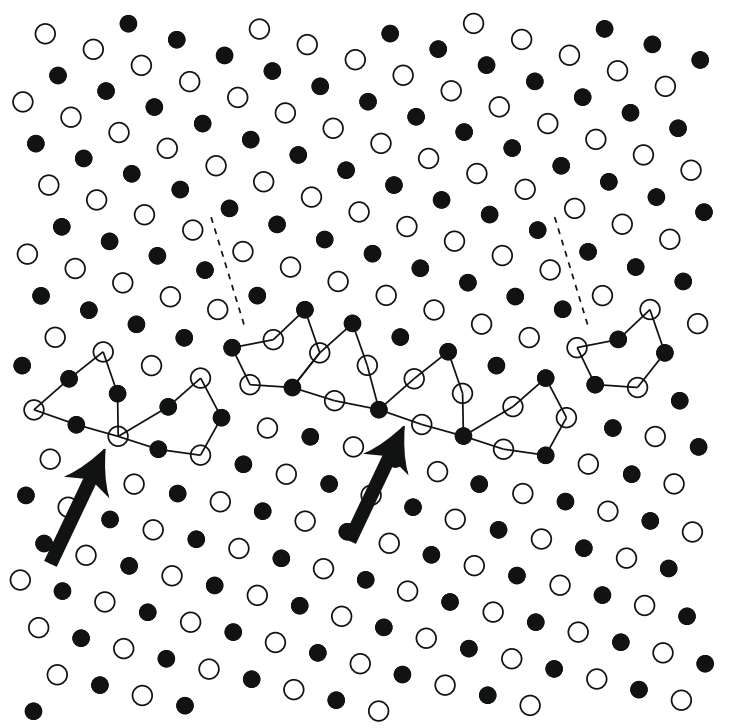

(b)

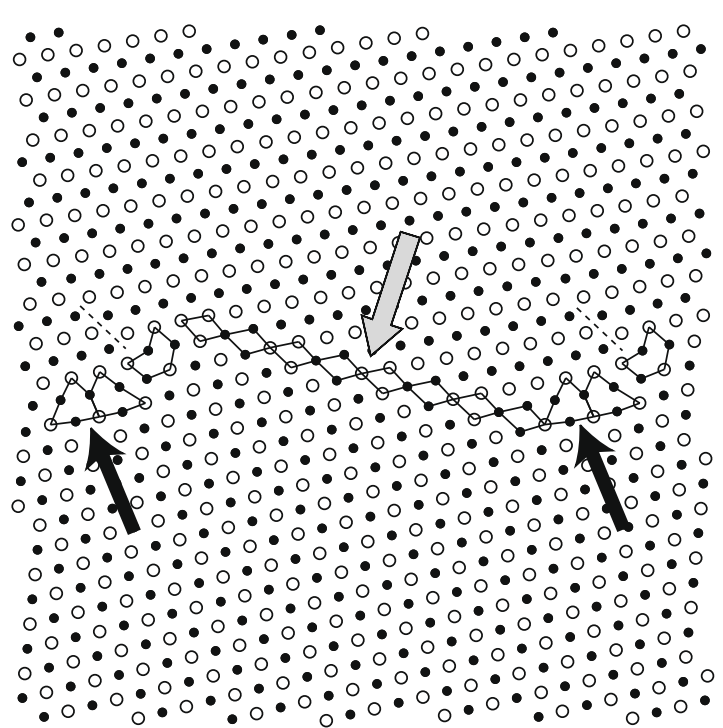

Fig. 3. Atomic structure of selected $\Sigma 11$ [1 10$]$ asymmetrical grain boundaries with inclination angles (a) $\alpha=46.7^{\circ}$ and (b) $\alpha=74.2^{\circ}[234]$. The structures are viewed along the tilt axis [1 10$]$, with the open and filled circles indicating atomic positions in alternate (220) planes. The gray arrow shows a facet with the structure of the $\Sigma 11$ [1 110$]$ (1 113 ) symmetrical tilt boundary. The black arrows show facets with the structure of an incommensurate non- $\Sigma 11$ boundary with a $(001) /(111)$ plane matching. The dashed lines indicate intrinsic stacking faults originating from the junctions of the nanofacets.

GB faceting is a generic phenomenon displayed by many materials $[12,216]$, in which an initially flat boundary spontaneously develops a sawtooth profile in a manner similar to phase transitions [12,224,226,239-241]. The faceting transition can lead to asymmetrical facets even if the average orientation of the boundary plane is symmetric, and vice versa. Hamilton et al. [226] applied first-principles, EAM and continuum elasticity methods to study the faceting transition at a $\Sigma 3(110)$ boundary in Al which had previously been observed by TEM [242]. They found that the equilibrium facet size grew as the cross-sectional area of the boundary increased, suggesting that finite-size facets were unstable unless the boundary plane was confined between surfaces, triple junctions or other defects. Their work has demonstrated a possible mechanism of the size effect on GB structures in confined systems such as nanograined materials, thin films and nanowires/pillars. The finite-size effects have been further explored by the same group by combining atomistic simulations with HRTEM observations on $\mathrm{Au}$ thin films [243].

More recently, the facet stability problem has been revisited by examining three different GBs in Al using two different EAM potentials [235]. The results suggest that the $\Sigma 3$ (110) faceting behavior reported in Ref. [226] presents only one possible case. In other cases, finite-size facets were found to be very stable and either did not grow during the simulations or grew extremely slowly. This stability against facet growth was explained by a high-energy barrier of facet migration arising due to large GB stresses. This barrier can lead to very slow kinetics of facet migrations, which is a necessary step of the coarsening process.

Another interesting effect found in some GBs is the formation of thin layers with a structure different from the crystal structure in the grains. For example, $\Sigma 3$ (211) $\mathrm{GBs}$ in $\mathrm{fcc} \mathrm{Cu}$ can form thin layers with a bcc structure, an effect that was predicted by atomistic simulations and confirmed by HRTEM observations [227,228].

\subsection{Grain boundary thermodynamics}

Thermodynamic properties of interfaces play an important role in capillarity-driven processes such as grain growth, precipitation and coarsening, as well as in solute segregation phenomena and interface decohesion. This is a vast area of research, in which atomistic simulations have been widely applied and have provided significant insights. The work before the mid-1990s has been thoroughly reviewed by Sutton and Balluffi [12]. We will discuss some of the most recent results, focusing on GBs.

Thermodynamic properties of GBs in single-component systems have been studied extensively by several different complementary methods based on the quasi-harmonic approximation [174,244-252] and thermodynamic integration techniques [174,245,246,250,251,253,254]. For detailed descriptions and comparisons of the results obtained by different methods, the interested reader is referred to Refs. [174,245,249-251].

The calculated GB free energies $(\gamma)$ in elemental crystals have generally been found to decrease with increasing temperature, by a magnitude that varies with the boundary type and the nature of the interatomic bonding. In a systematic study of [1 00] twist boundaries in $\mathrm{Au}$ [247], the values of $\gamma$ were found to decrease by as much as $25 \%$ at temperatures from 0 to $700 \mathrm{~K}$. It was demonstrated that the interface entropy can induce cusps in $\gamma(\theta)$ at high temperatures for misorientations $\theta$ distinct from those corre- 
sponding to the zero-temperature GB energy. For [100] symmetrical tilt boundaries in fcc Lennard-Jones and $\mathrm{Cu}$ crystals, $\gamma$ was found to decrease by as much as a factor of two as the temperature increased from $0 \mathrm{~K}$ to the melting point $[245,251]$. For these systems, large increases in the magnitude of $(\partial \gamma / \partial T)_{P}$ occur at homologous temperatures larger than approximately 0.5 , and are correlated with the onset of pronounced structural disorder within the boundary [245]. In detailed calculations of interfacial free energies for twin boundaries in $\mathrm{Si}$, modeled with the StillingerWeber potential [158], $\gamma$ was found to decrease by less than $10 \%$ relative to the $0 \mathrm{~K}$ value, at a homologous temperature of approximately 0.6 [250].

In the context of grain growth, the thermodynamic driving force for GB motion is governed by the GB stiffness, the quantity entering into the Gibbs-Thomson equation for the chemical potential jump across a curved interface when $\gamma$ is anisotropic [255-257]. When the GB has a nonzero curvature in only one principal direction, the stiffness can be written as $\Gamma=\gamma+\gamma^{\prime \prime}$, where $\gamma^{\prime \prime}=\partial^{2} \gamma / \partial \phi^{2}$ is the second derivative of $\gamma$ with respect to variations in the orientation $(\phi)$ of the boundary normal (see Ref. [257] for a detailed discussion of interface stiffness in three dimensions). Calculations of GB stiffness by the methods mentioned above are complicated by the need for an accurate parameterization of the dependence of $\gamma$ on misorientation, in order to compute $\gamma^{\prime \prime}$. An alternative approach, applicable at temperatures above the roughening temperature of the boundary, involves extracting the stiffness directly from an analysis of equilibrium capillary fluctuations in MD simulations $[258,259]$. From an analysis of the power spectrum of the Fourier amplitudes describing the fluctuations in interface position, $\Gamma$ can be extracted for curvatures along different crystallographic directions, as illustrated in Fig. 4. As with the GB free energy, the stiffness is seen to decrease with increasing temperature. An important result is the large variation in the stiffness for different directions of the GB curvature [259]. Further comments on this method and its application to GB mobility can be found in Section 9.5.

\subsection{Grain boundary segregation and embrittlement}

Solute segregation at GBs is another important aspect of interface thermodynamics, which is closely related to mechanical behavior of materials through the embrittlement effect (see also Section 6.4). Some impurities, such as $\mathrm{H}, \mathrm{O}, \mathrm{S}$ and $\mathrm{P}$, strongly segregate to GBs and reduce their cohesion, rendering otherwise ductile metals extremely brittle. Whether such elements are introduced during fabrication or penetrate from the environment, their detrimental effect on ductility and toughness can be dramatic. Other impurities (e.g. B and C) can have a beneficial effect by increasing GB cohesion or by expelling harmful elements from the GB by site competition.

First-principles calculations have been very efficient in predicting the embrittling potency of various elements and elucidating the physical mechanisms of the impurity effects. The most common approach is to compare binding energies of the impurity atoms to the GB and to the surface that would form by cleaving the material along the boundary. It is expected [260] that embrittling impurities should have a stronger binding to the surface than to the GB, with an opposite trend for GB-strengthening elements. In a series of first-principles calculations based on this criterion, $\mathrm{H}$, $\mathrm{O}, \mathrm{P}$ and $\mathrm{S}$ were predicted to cause GB embrittlement while $\mathrm{C}, \mathrm{B}$ and $\mathrm{N}$ were predicted to cause a strengthening effect in bcc Fe [261-264]. Boron can actually be either an embrittler or a cohesion enhancer in $\mathrm{Fe}$, depending on the site occupation [264]. An increasing amount of segregation can further enhance the embrittlement due to repulsive interactions between the impurity atoms (e.g. S or P), which further destabilize the boundary relative to the surface $[265,266]$. Similar results were obtained for the mentioned elements in Ni [267-269] (see [265] for a comprehensive review), as well as for $\mathrm{B}, \mathrm{C}, \mathrm{N}$ and $\mathrm{O}$ in $\mathrm{Mo}$ and $\mathrm{Nb}$ [270]. Despite the use of relatively small supercells with simple $\Sigma 3$ or $\Sigma 5 \mathrm{GBs}$, the predicted embrittlement trends are in good agreement with experimental results. These trends were mostly attributed to electronic effects, such as charge transfer towards the impurity atoms resulting in weakening of the bonding between neighboring host atoms $[262,267,269,271,272]$.

The embrittlement of $\mathrm{Cu}$ has recently received special attention in connection with the $\mathrm{Bi}-\mathrm{Cu}$ system, in which very small amounts of $\mathrm{Bi}$ can produce catastrophic GB embrittlement. In recent years, this system essentially became the testing bed for atomic-level theories of embrittlement and eventually the subject of debate and controversy. Duscher et al. [273] performed electronic structure calculations for $\mathrm{Bi}$ atoms occupying a certain (relatively open) substitutional site in the $\Sigma 5(310)[001]$ GB. They arrived at the conclusion that $\mathrm{Bi}$ atoms donate some extra charge to surrounding $\mathrm{Cu}$ atoms, making their electronic structure "Zn-like". The reduction of the $d$-like density of states near the Fermi level reduces the directional character and strength of $\mathrm{Cu}$ bonds, resulting in GB weakness. These conclusions were supported by experimental analysis of the electronic structure of the $55 \mathrm{~GB}$ with segregated Bi atoms using electron energy loss spectroscopy [273].

Other authors [274-276] disagreed with this model based on their own first-principles calculations for $\mathrm{Bi}$, $\mathrm{Na}, \mathrm{Ag}$ and $\mathrm{B}$ in $\Sigma 5$ and $\Sigma 19$ GBs. They performed a careful, step-by-step analysis that separated the chemical and structural effects. Their conclusion was that the electronic effects cannot be responsible for the Bi embrittlement, and in fact would strengthen, not weaken, $\mathrm{Cu}$ bonding. Instead, the embrittlement was explained by the mere size effect, in which the strongly oversized $\mathrm{Bi}$ atoms push $\mathrm{Cu}$ atoms apart and weaken their bonding. The calculations $[274,275]$ have shown that at least a monolayer of $\mathrm{Bi}$ in GBs is needed to produce the embrittlement, suggesting that the particular GB studied in Ref. [273] was in fact not embrittled. A strong embrittling effect by the same 

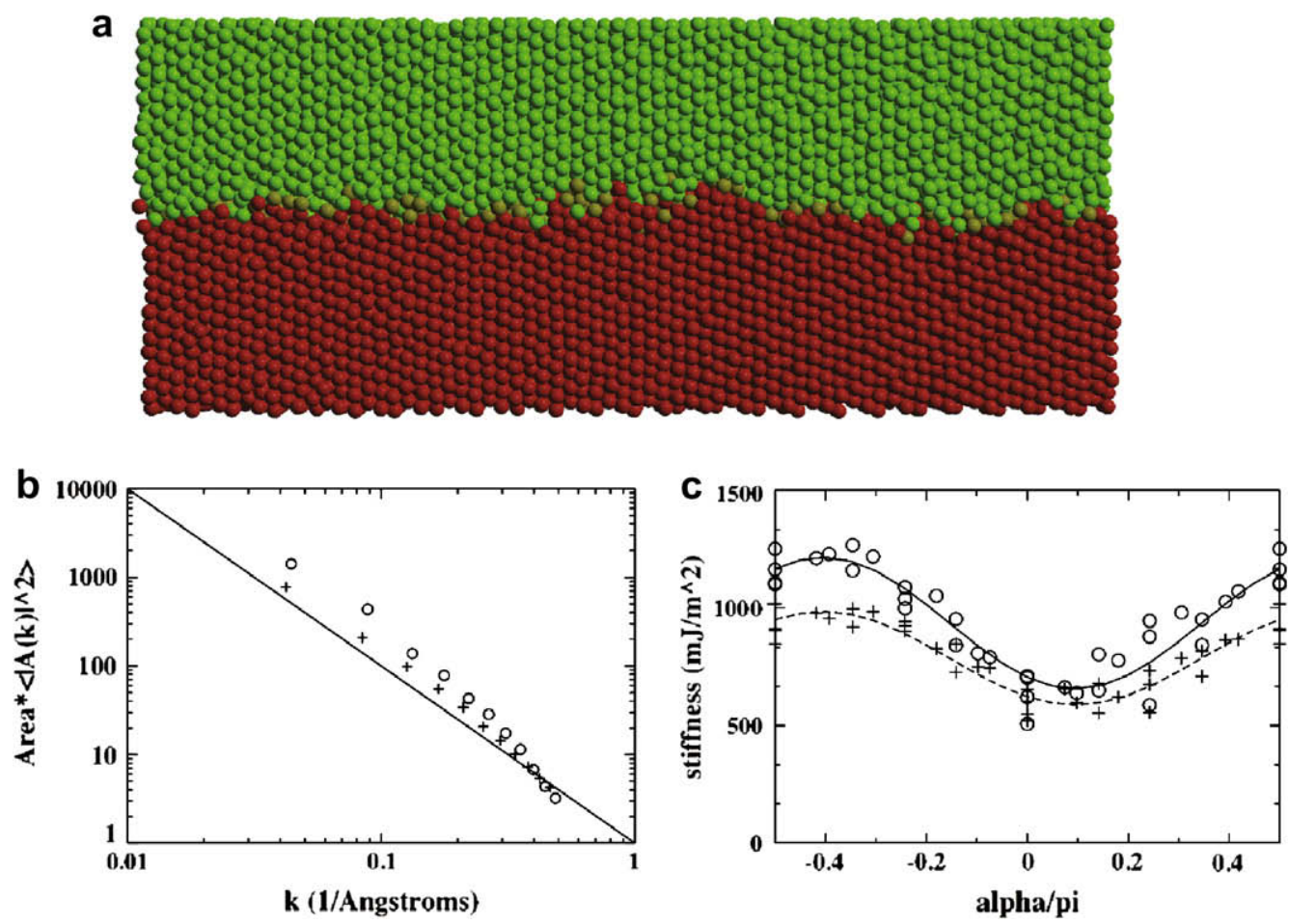

Fig. 4. An illustration of the capillary fluctuation method to compute grain-boundary stiffness, reproduced from Ref. [259]. The snapshot (a) is taken from an MD simulation for an asymmetric $\Sigma 7[111]$ tilt boundary in $\mathrm{Ni}$ at $T=1400 \mathrm{~K}$. The atoms are colored by an order parameter designed to identify whether the local geometry is consistent with one grain or the other. The non-planar nature of the boundary (the interface between the green and red atoms) is apparent. A plot of the averaged square Fourier amplitudes for the interface position are plotted in (b) vs. wavenumber on a log-log plot; the solid line gives the theoretical slope of $k^{-2}$. From a fit of the MD data to the theoretical formulas for equilibrium capillary fluctuation spectra the GB stiffness can be calculated for different directions of the interface curvature. This is shown in (c), where the open circles and crosses correspond to results at $1250 \mathrm{~K}$ and $1400 \mathrm{~K}$, respectively.

mechanism was predicted for $\mathrm{Na}$, whereas $\mathrm{Ag}$ was predicted not to embrittle $\mathrm{Cu}$ [275], both predictions being in agreement with experimental results. Boron was found to segregate by a combination of interstitial and substitutional mechanisms. Its influence on the GB strength is believed to be a combination of chemical and size effects [276].

$\mathrm{Ga}$ in $\mathrm{Al}$ is another prominent case of severe GB embrittlement that has been studied by first-principles methods. Thomson et al. [277,278] investigated the role of Ga by placing different amounts of $\mathrm{Ga}$ atoms at different positions in the $\Sigma 11$ (311) Al boundary. They found that Ga strongly prefers "tight" sites in the GB structure and attributed them the key role in the Ga behavior. They proposed a qualitative explanation of the $\mathrm{Ga}$ embrittlement effect based on the notion of "two radii" of Ga atoms, a nearest-neighbor radius and a volume radius. A more recent study of $\mathrm{Ga}$ in the $\Sigma 9$ (221) Al boundary [279] suggests that $\mathrm{Ga}$ atoms draw charge from neighboring $\mathrm{Al}$ atoms, reducing the charge density on $\mathrm{Al}-\mathrm{Al}$ and $\mathrm{Al}-\mathrm{Ga}$ bonds. This charge reduction is enhanced by increasing amounts of $\mathrm{Ga}$ segregation and may be responsible for the GB embrittlement. On the other hand, investigations of $\mathrm{Al}$ surfaces covered with $\mathrm{Ga}$ [280] suggest that $\mathrm{Ga}$ should strongly embrittle even single-crystalline $\mathrm{Al}$, which is consistent with experimental observations.
Although our discussion is focused on metals, it should be mentioned that atomic-level modeling has been actively applied to study segregation and its effect on mechanical behavior in ceramic materials as well. As a recent example combining first-principles calculations with HRTEM observations, Buban et al. [281] were able to resolve the atomic structure of the $\Sigma 31 \mathrm{~GB}$ in alumina with and without $\mathrm{Y}$ dopants, and were able to identify the particular structural units featuring a preferential occupation by $\mathrm{Y}$ atoms. The GB structures computed with VASP were in good agreement with HRTEM and confirmed the energetic preference of the experimentally observed $\mathrm{Y}$ positions. A detailed examination of the $\mathrm{Y}$ effect on the bonding strength in the GB region has provided electronic insights into the dramatic improvement of creep resistance of alumina by Y-doping.

Because segregation and embrittlement studies heavily involve chemical effects, interatomic potentials have been less useful overall than first-principles methods. Nevertheless, EAM-based MD and MC simulations have been applied for generic investigations of structural and thermodynamic aspects of interface segregation [282-293]. In particular, Millet et al. [293] performed a parametric study of the effect of oversized impurities with various radii on GB segregation in polycrystalline $\mathrm{Cu}$ samples. They were able to demonstrate a stabilizing effect of the impurities on the 
GB structure by a reduction in the interface free energy and thus the driving force for the grain growth.

\subsection{Structure and thermodynamics of solid-solid heterophase interfaces}

Atomic-scale modeling has been applied extensively to solid-solid heterophase interfaces. A thorough review of this topic is beyond the scope of this paper, and what follows will necessarily be focused on only a few examples for metal/metal heterophase interfaces relevant in the context of alloy microstructures. Further, we will focus on structural and thermodynamic properties of such interfaces, highlighting primarily the use of first-principles DFT-based methods. Solid-solid heterophase interfaces are ubiquitous in commercial alloys, and their properties play an important role underlying the stability and morphology of microstructures and the associated mechanical properties.

Fig. 5 illustrates an application of DFT calculations to interfaces between the $\alpha$-Al matrix and $\beta^{\prime \prime} \mathrm{Mg}_{5} \mathrm{Si}_{6}$ precipitates in an Al-Mg-Si alloy [294]. For interfaces of this type, involving relatively complex precipitate phases, central questions concerning interface structure include the orientation relationships between the phases, their relative displacements parallel to the interface plane and their preferred crystal-plane terminations. Fig. 5 illustrates the size and the supercell geometries that can be readily employed in modern DFT calculations; the computational speed

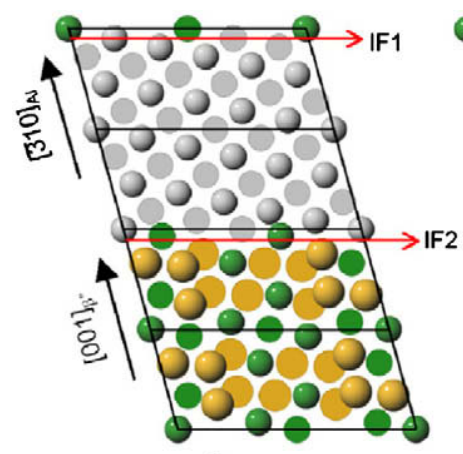

$\mathrm{C}_{1}$

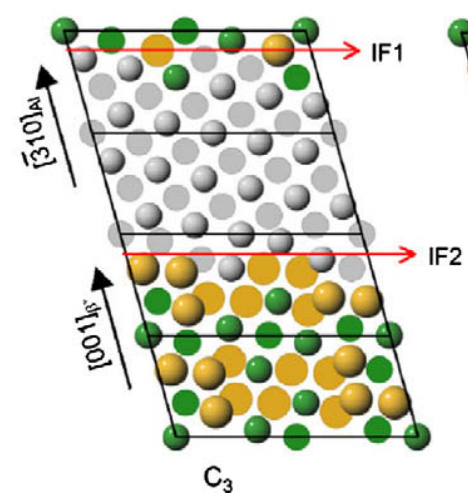

$\mathrm{C}_{3}$

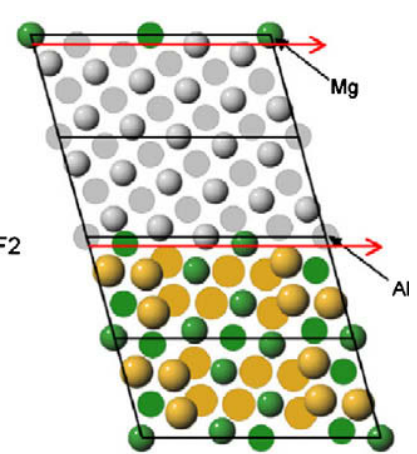

$\mathrm{C}_{2}$

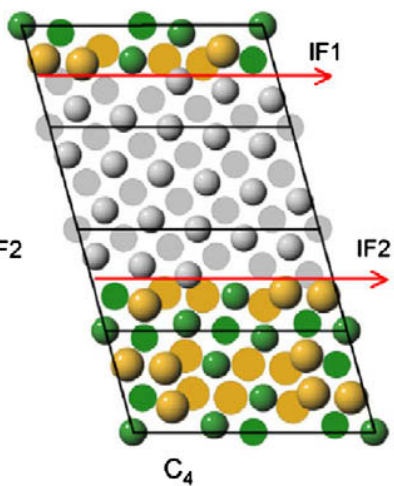

$\mathrm{C}_{4}$
Fig. 5. Supercells used in calculating the interfacial energies between $\alpha$-Al and $\beta^{\prime \prime}-\mathrm{Mg}_{5} \mathrm{Si}_{6}$ phases in $\mathrm{Al}-\mathrm{Mg}-\mathrm{Si}$ alloys [294]. The reader is referred to the original reference for a detailed explanation of the figure. associated with structural optimizations of models of this size is sufficiently fast that the energy of many different orientations and atomic configurations can be screened to arrive at plausible low-energy structures, as demonstrated in Ref. [294] (see also Ref. [295]). An important output of such calculations is the magnitude and anisotropy of the associated interfacial energies. These energies are important input parameters required for mesoscopic simulations of precipitate growth kinetics, and they are often not experimentally accessible. An example demonstrating the use of first-principles calculations to provide interfacial energies as input for quantitative phase-field modeling of precipitate growth morphologies can be found in Refs. [296,297].

For semicoherent or incoherent interfaces, the direct first-principles approach described above is limited to systems where the lateral periodicity in the interface plane is sufficiently small for the structure to be modeled by a few hundred atoms. If this is not the case, another option is to employ interatomic potentials [298], which give access to much larger models than can be handled by first-principles methods. Many examples of studies of semicoherent metal-metal interfaces have been reviewed in Ref. [298] (see [142] for a more recent example). When potentials cannot accurately describe the chemistry of the system, hybrid approaches have been employed combining first-principles supercells with continuum elasticity models to parameterize separate contributions to the interface energy arising from chemical, strain and misfit-dislocation contributions. Applications of this approach for metal/carbide interfaces can be found in Refs. [298,299].

Another complication arises when the interfaces are not compositionally sharp. In multicomponent systems, solute atoms may preferentially segregate to heterophase interfaces and, as for GBs, can give rise to substantial changes in structural, thermodynamic and cohesive interfacial properties. When the solute concentrations are dilute, equilibrium segregation to heterophase interfaces can be predicted relatively straightforwardly within a first-principles framework by computing the formation energy of single solute atoms at different positions in the vicinity of the interface. By employing these energies within standard mean-field statistical-thermodynamic models for dilute solutions, equilibrium composition profiles and associated changes in interfacial free energies can be calculated. Examples illustrating this approach can be found for Al$\mathrm{Mg}-\mathrm{Sc}$ alloys [300,301] and Ni-based superalloys [302308]. For systems where atom-probe data are available [300,301,303,304,308], first-principles predictions for solute segregation have been found to agree well with experimental results. Such calculations have also been used to examine the effects of solute segregation on interfacial free energies [301,304] and interface cohesion [307,309-312].

The dilute-solution models described above are not applicable to concentrated alloys. Atomistic simulations have been applied extensively in the modeling of compositionally diffuse coherent interfaces in concentrated alloys, employing one of two approaches. The first involves the 
CE formalism described in Section 2.2. In this approach, a first-principles-based cluster expansion for the alloy energetics is coupled with MC simulations or the clustervariation method to compute temperature-dependent composition profiles and interfacial free energies (see examples in Refs. [313-318]). The second approach employs grand-canonical MC simulations with interatomic potentials $[104,319,320]$. An example of an equilibrium composition profile calculated by this approach for an $\mathrm{Ni} / \mathrm{Ni}_{3} \mathrm{Al}$ (100) interface at $700 \mathrm{~K}$ is shown in Fig. 6 [104]. The interface is seen to be compositionally diffuse, with the envelope of the Al concentration profile varying between its bulk values in the $\gamma^{\prime}$ and $\gamma$ phases across several atomic planes. A similar result for $\mathrm{Ni} / \mathrm{Ni}_{3} \mathrm{Al}$ interfaces at $1000 \mathrm{~K}$ was obtained using a CE approach in Ref. [317]; this reference also discusses an important consequence of such diffuse composition profiles on diffusion-mediated coarsening kinetics. The formation of diffuse composition profiles has also been shown to correlate with a large reduction in the magnitude and anisotropy of coherent interfacial free energies with temperature [313-315,321]. For example, in $\mathrm{Al}-\mathrm{Li}$ alloys the $\alpha / \delta^{\prime}\left(\mathrm{fcc} / \mathrm{L}_{2}\right)$ interfacial free energies decrease in magnitude by as much as a factor of 5 (for the highest-energy orientation) as the temperature is raised from 0 to $500 \mathrm{~K}[313,321]$. This reduction in the calculated values was critical to achieving good agreement with experimental estimates based on precipitate coarsening measurements.

\subsection{Structure and thermodynamics of solid-liquid interfaces}

In the nucleation and growth of a crystal from its melt, the properties of solid-liquid interfaces often play a critical role underlying phase selection and the formation and evolution of microstructure [322-324]. Atomistic simulations have found extensive applications as a framework for prob-

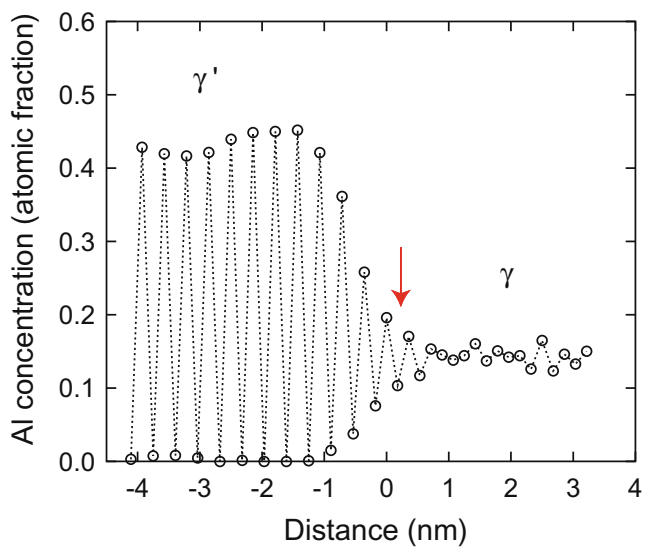

Fig. 6. Composition profile across the coherent $\gamma / \gamma /$ (100) interface in the $\mathrm{Ni}-\mathrm{Al}$ system obtained by Monte Carlo simulations at $700 \mathrm{~K}$ [104]. The points represent the average atomic fraction of $\mathrm{Al}$ atoms in individual (200) planes parallel to the interface. The arrow shows the initial interface position at $0 \mathrm{~K}$. ing the structure, thermodynamic and kinetic properties of such interfaces, and have been used to derive input parameters to phase-field simulations of solidification [325,326]. To date, most atomistic studies have focused on crystalmelt interfaces in single-component systems. This work has included detailed analyses of interfacial structure, as well as direct calculations of interfacial free energies and kinetic coefficients, and their associated crystalline anisotropies. Comprehensive reviews of the methods employed in these studies, and their application to pure metals and related model systems can be found in Refs. [325-328]. In this section we highlight applications related to equilibrium solid-liquid interfaces in alloys and chemically heterogeneous systems.

Detailed studies of crystal-melt interface structures have been undertaken for alloy systems based on hard-sphere [329-332] and Lennard-Jones potentials [333,334], and EAM potentials for $\mathrm{Cu}$ [335], an $\mathrm{Ni}-\mathrm{Cu}$ alloy [336] and the $\mathrm{Cu}-\mathrm{Ag}$ system [337]. Equilibrium solute and solvent density profiles have been calculated, and the magnitudes of relative solute adsorption coefficients have been computed to have values on the order of a few percent per interface site or less in most cases [330,332-334,336,337] (a notable exception was found in Ref. [334] at temperatures where the solute densities in solid and liquid phases were nearly equal).

Only relatively recently have simulation methods been demonstrated for calculating solid-liquid interfacial free energies $\gamma$ in alloys [334,338-340], through the use of the capillary fluctuation method [341] briefly described in the context of GB stiffness in Section 5.2. This method has been used in two recent studies to investigate the effect of solute additions on the magnitude and crystalline anisotropy of $\gamma$ in model fcc-forming mixtures [334,339,340]. Amini and Laird [340] considered a hardsphere mixture with size-ratio of 0.9 , while Becker et al. [334,339] studied a Lennard-Jones alloy featuring no atomic size mismatch and a ratio of elemental melting temperatures equal to 0.75 . In both cases, it was found that $\gamma$ decreases with increasing concentration of the lower-melting-point species (at fixed pressure). In addition, the anisotropy of the interfacial free energy was characterized by the relative ordering $\gamma_{100}>\gamma_{110}>\gamma_{111}$ for the high-symmetry [1 00$],\left[\begin{array}{lll}1 & 1 & 0\end{array}\right]$ and [1 111$]$ interface orientations, at all concentrations. An alternative method of calculation of $\gamma$ by thermodynamic integration has been proposed in Ref. [337].

Although the anisotropies in $\gamma$ are relatively small, being on the order of a few percent for atomically rough fcc crystal-melt interfaces, they can have important consequences for dendritic solidification microstructures, as discussed in Ref. [339,342]. For Lennard-Jones alloys, addition of solute was shown to give rise to a tendency towards destabilizing the $\langle 100\rangle$ orientation for dendrite growth, while no such effect was found for the hardsphere mixtures. These studies highlight a clear need for further atomistic investigations to better understand the 
nature of the microscopic interactions of solute atoms with solid-liquid interfaces, and the ways in which these interactions affect interface properties and thus influence solidification microstructures on larger scales.

The least studied class of solid-liquid interfaces are those between chemically dissimilar crystal and liquid phases (e.g. liquid $B$ against solid $A$ ). Such interfaces are particularly relevant in the context of heterogeneous nucleation, wetting phenomena and catalyst-mediated nanowire growth, yet their equilibrium thermodynamic properties have not been studied in great detail. For such heterogeneous solid-liquid systems, the simulation studies to date [343-346] have focused primarily on the investigation of equilibrium interface structures, diffusion and the formation of highly ordered structures in the liquid layers adjacent to the crystal. A recent review of this topic can be found in Ref. [346].

\section{Mechanical response of interfaces}

\subsection{Interaction of interfaces with dislocations}

Although interactions of interfaces with dislocations play an important role in mechanical behavior of materials, many aspects of this process are not well understood. In particular, slip transfer across GBs is important in deformation behavior of nanocrystalline materials, thin films and multilayers (Section 7).

Molecular statics simulations with EAM potentials were employed to study dislocation interaction with coherent twin boundaries in fcc metals [347,348]. Under an applied shear stress, a perfect screw dislocation could either transmit though the boundary or dissociate into partials in the boundary plane, depending on the generalized stacking fault energy and other material-dependent parameters. For an incident mixed $\left(60^{\circ}\right)$ dislocation, the dissociation reactions at the boundary were more complex and resulted in partials gliding into the neighboring twin and leaving a locked configuration in the boundary. Similar conclusions were reached in simulations of the impingement of single and multiple screw dislocations on various tilt GBs in Al using a multi-scale modeling approach [349]. Slip transmission through high-symmetry $\Sigma 3$ and $\Sigma 11$ boundaries was very difficult even after the head dislocation was fully absorbed and a pile-up formed in one grain. On the other hand, transmission through a $\Sigma 9$ boundary with a more complex atomic structure readily occurred by absorption of screw dislocations on one side and nucleation of new dislocations on the other side. This process depends very sensitively on the orientation of the applied stress and on the precise location where the dislocations enters the boundary.

Similar methods were applied to model dislocation interactions with symmetrical tilt GBs in bcc metals [350,351]. A lattice dislocation absorbed by the boundary created a highly distorted boundary region, which gave rise to a stress concentration and favored a crack nucleation.
The stress needed for the crack nucleation depended sensitively on the type of the load (e.g. uniaxial vs. biaxial), orientations of slip systems in the grains relative to the boundary plane, the detailed GB structure and many other factors. In some cases, the crack nucleation was preceded by emission of several dislocations into the grains. These simulations demonstrate that the crack nucleation process at GBs is more complex than often described in terms of a simple pile-up model.

Extensive work has been done on the slip resistance of GBs and heterophase interfaces in multi-layer systems. Atomistic simulations of coherent and semi-coherent fcc/fcc interfaces [352,353] indicate that the main factor in their resistance to slip transfer comes form the alternating tension-compression coherency strain regions. Incoherent interfaces present stronger barriers to slip transfer due to their relatively weak sliding resistance. The easy interface sliding produces image forces that attract glide dislocations into the interface. Once captured, the dislocation delocalizes its core and a new dislocation has to be nucleated in order to transmit the slip into the other phase. Attractive interaction with dislocations is also found for GBs. The interaction of screw dislocations with coherent twin boundaries in $\mathrm{Cu}$ and $\mathrm{Al}$ changes from repulsive at large distances to attractive at short distances, resulting in dislocation absorption by the boundary [354].

Similar trends were observed for fcc/bcc interfaces between different phases. Hoagland and co-workers [355357] have conducted extensive simulations of slip transfer across such interfaces in multi-layer composites. Their work has demonstrated the critical importance of the shear strength of interfaces for their interaction with lattice dislocations. If the shear strength is low, the stress field of a glide dislocation approaching the interface shears it locally, producing an image force that attracts the dislocation. This attraction results in the dislocation absorption and core spreading in the interface. For the dislocation transmission to the next phase, the dislocation must compact its core, which is a difficult process, making the interface a strong barrier to slip.

These effects were especially clearly observed in detailed studies of the shearing process of plane $\mathrm{Cu}-\mathrm{Nb}$ interfaces with the Kurdjumov-Sachs orientation relationship [355]. The shear resistance was found to be anisotropic and relatively weak, leading to an easy dislocation spreading in the interface and its high resistance to slip transmission. The interface sliding was mediated by the nucleation and spreading of interface dislocation loops, leading to a serrated behavior of the shear stress as a function of strain. Hoagland et al. [355] emphasize the significant difference between the slip resistance of coherent or semi-coherent interfaces, on the one hand, and that of incoherent interfaces on the other. In the former case, the slip planes are nearly continuous across the interface and glide dislocations can be transmitted through it. In the latter case, the slip can only continue by renucleation of new dislocations. 


\subsection{Interface deformation and sliding}

Interfaces are often the sites of damage initiation under applied mechanical loads. Substantial insight into the mechanisms of plastic deformation and fracture nucleation at interfaces has been obtained by atomistic methods. Possible responses of an interface to applied loads include interface sliding, interface migration coupled to shear deformation, dislocation emission in the grains/phases, and crack nucleation and propagation. In this section we discuss dislocation emission and sliding, followed by coupled GB migration (Section 6.3) and GB fracture (Section 6.4).

In a series of papers [358-360], sliding along a high-symmetry $\Sigma 11$ [ 1110$]\left(\begin{array}{lll}1 & 1 & 3\end{array}\right)$ tilt GB in aluminum was studied by molecular statics. As expected, a perfect GB with the ground-state structure exhibited an extremely high sliding resistance. Extrinsic defects, such as small steps and partial dislocations, reduced the critical stress of sliding by orders of magnitude. Disconnections (interface defects possessing both step and dislocation character [361,362]) could inject partials into one of the grains, contributing to the accommodation of the stresses arising during GB sliding and migration. An impingement of lattice dislocations on the GB produced a chain of defect reactions resulting in glissile defects which facilitated both GB sliding and migration. A number of interesting dislocation reactions were found in which the GB served as a source of lattice dislocations.

Sansoz and Molinari [229,363] performed simulated mechanical tests of several symmetrical and asymmetrical [1 110$]$ tilt $\mathrm{GBs}$ in $\mathrm{Cu}$ for shear and tension using quasitwo-dimensional (2-D) MD simulations combined with a quasi-continuum method. The boundaries responded to applied loads by sliding, coupled motion or emission of partial dislocations into the grains, depending on the grain misorientation and the GB plane. The authors found a correlation between the easy sliding observed in some of the boundaries and the occurrence of a certain type of structural units, called E-units. Such units are represented by capped trigonal prisms in three dimensions and are common structural units in many tilt boundaries. An interesting observation was the stick-slip behavior of the shear stress during the sliding processes [229,363].

MD simulations were employed to study the effect of applied tensile stresses on $\langle 100\rangle$ and $\langle 110\rangle$ symmetrical tilt GBs in aluminum and copper $[230,364,365]$. The mechanical failure along the boundaries was initiated by partial dislocation emission into the grains, accompanied by atomic rearrangements in the boundary. Such rearrangements often resulted in the formation of ledges which gave rise to a stress concentration. The tensile strength of the boundary depended on the orientation of the tensile load relative to the boundary plane and on certain structural features of the boundary, including the existence of the E-units mentioned above and distribution of free volume. A nonSchmidt behavior of the interface strength was observed, in which the dislocation nucleation events were signifi- cantly influenced by stress components normal to the slip planes.

\subsection{Grain boundary motion coupled to shear deformation}

GB motion coupled to shear deformation is an area where atomistic methods have been especially productive [366-370]. Stress-induced GB motion was observed in first-principles calculations [371-373] and atomistic simulations [229,374-377], as well as in experiments on low-angle [378,379] and more recently high-angle [380-389] GBs. That the coupling effect might be a generic property of most GBs was proposed in the Cahn-Taylor "unified approach" paper [390] based on theoretical considerations. Since then, significant progress has been made in understanding the atomic mechanisms, geometric rules and dynamics of coupled GB motion, and its relation to other processes in materials [366-370,387,391,389].

The basic idea of the coupling effect is that a shear stress applied to a GB induces its normal motion (Fig. 7). In turn, GB motion induced by an applied shear stress, or by any other driving force, produces shear deformation of the volume swept by the motion. Because this deformation is usually a simple shear parallel to the GB plane, the GB motion is coupled to grain translations. It has also been shown that the coupled motion of a curved GB induces grain rotation [390,392,393], which, in turn, creates a driving force for normal GB motion. Thus, a number of seemingly disparate phenomena originate from essentially the same physical effect. It should be emphasized that the coupled state of a boundary exists independently of applied driving forces. It has been demonstrated [368,370], for example, that spontaneous GB displacements caused by thermal fluctuations can produce shear deformations that follow all geometric rules of coupling. The coupling effect is characterized by a factor $\beta$ equal to the ratio of the tangential grain translation to the associated normal GB displacement or, equivalently, the ratio of the tangential and normal velocities, $v$ and $v_{n}$. The coupling is called perfect if $\beta=v / v_{n}$ is a geometric constant that depends only on the GB bicrystallography and not on the GB velocity or driving force.

Coupled GB motion is now recognized to be a very common phenomenon, existing due to the atomically ordered structure of GBs. Simulations have identified dozens of coupled GBs [366-368,370,393,394]. Stress-induced GB motion was observed in experiments on bicrystals in both metals [382-384,387-389,395-397] and ceramic materials [386], with experimental coupling factors matching their perfect geometric values [395-397]. The coupling effect might be responsible for the stress-driven GB motion and stress-induced grain growth in nanocrystalline materials [200,387,398-400].

The coupled GB motion does not require any diffusion and is implemented by deformation and rotation of structural units forming the boundary. The unit transformations occur by a nucleation and growth mechanism in which the transformed GB area is bounded by a growing disconnec- 
(a)

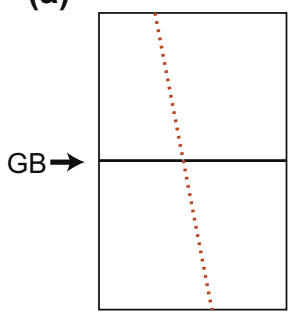

Initial GB

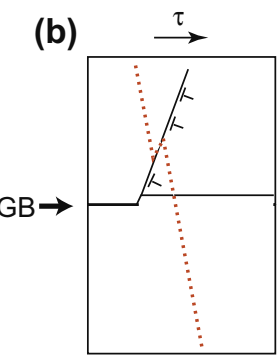

Slip initiation

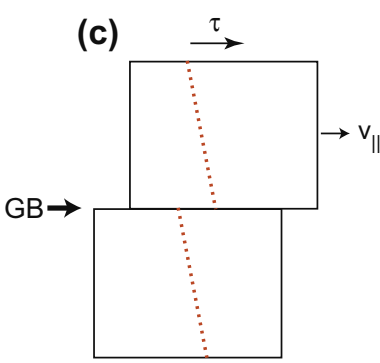

GB sliding

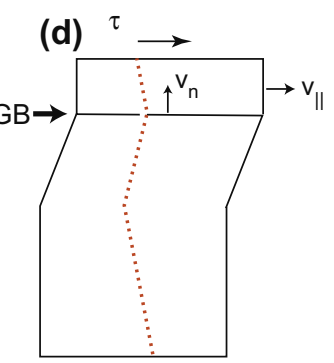

Perfect coupling

Fig. 7. Possible mechanical responses of a plane GB to applied shear stresses $\tau$. (a) Initial bicrystal with a dotted line showing a set of inert markers. (b) The GB initiates slip by emitting a dislocation. (c) Rigid GB sliding with a grain translation velocity $v_{\|}$; note the discontinuity of the marker line. (d) GB motion coupled to shear deformation ( $v_{n}$ is the normal GB velocity).

tion loop. Some GBs are prohibited from coupled motion by symmetry, such as pure twist GBs [368]. Aside from such special cases, any ordered GB should be able to couple to shear stresses and move, unless other competing mechanisms (such as GB sliding or emission of dislocations) are activated more easily. Simulations have shown that coupled motion can be blocked by constraints imposed by triple junction lines and other defects [401].

By crystal symmetry, multiple coupling modes can be implemented in a given GB, each characterized by a different $\beta$ value, atomic mechanism and critical stress [366-368]. The active mode depends on the geometric parameters of the boundary, the orientation of the applied shear stress and its magnitude relative to the critical resolved shear stresses of different coupling modes [368]. The critical stresses of the coupling modes vary with temperature and their crossovers can produce mode switches. Although it should be possible to identify all geometrically allowed coupling modes of a given GB from its five macroscopic parameters and the point-symmetry group of the crystal, such a general theory is yet to be developed.

Another important finding of the simulations is that many GBs lose their ability to couple to stresses at high temperatures [368] and respond to applied stresses by rigid sliding without any normal motion. A transition from coupling to sliding usually occurs continuously over a temperature range until coupling completely disappears. There are two possible mechanisms for this transition. In one, the GB structure becomes highly disordered (e.g. due to premelting, Section 8.1), which precludes the deformation and rotation of the structural units. In other cases, the GB structure remains well ordered and is able to support coupled motion, but the temperature activates sliding mechanisms with lower critical stresses, making sliding the dominant response to the applied stress. Fig. 8 shows an example of a temperature-misorientation diagram of mechanical responses of [ $\left[\begin{array}{lll}0 & 0 & 1\end{array}\right]$ symmetrical tilt GBs in copper [368].

Simulations have also provided important insights into the dynamics of stress-driven GB motion. At low temperatures and relatively high velocities, the motion exhibits stick-slip behavior characterized by a saw-tooth time dependence of the stress and a stop-and-go character of the motion (Fig. 9). The magnitude of the peak stress increases with GB velocity $v$ but decreases with temperature $T$. Qualitatively, this happens because thermal fluctuations help the GB overcome the nucleation barrier before it vanishes, resulting in a lower value of the effective peak stress. A one-dimensional model of GB motion was proposed [369,370], representing the boundary by a particle attached to an elastic rod dragging the particle through a periodic potential. One of the predictions of this model is that, in the stick-slip regime, $\log v$ must be proportional to $\left(\tau_{c}^{0}-\tau_{c}\right)^{3 / 2} / k_{B} T$, where $\tau_{c}$ is the critical resolved shear stress at the simulated temperature and $\tau_{c}^{0}$ is its value at $0 \mathrm{~K}$. Thus, at a fixed velocity, the critical stress is expected to decrease with temperature as $T^{2 / 3}$, a relation which was

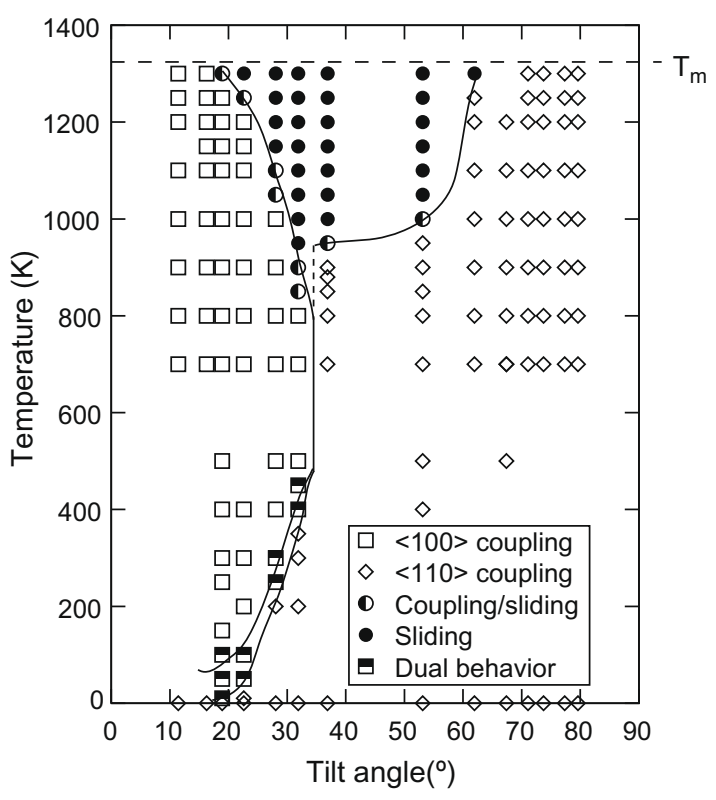

Fig. 8. Diagram of mechanical responses of [00 1$]$ symmetrical tilt GBs in copper obtained by MD simulations [368]. The points represent individual MD runs. In cases of dual behavior, the GB starts moving in the [110] coupling mode but later switches to the $[100]$ mode. Note that for highangle GBs coupling is replaced by sliding at about 0.7 of the melting point $T_{m}$. 

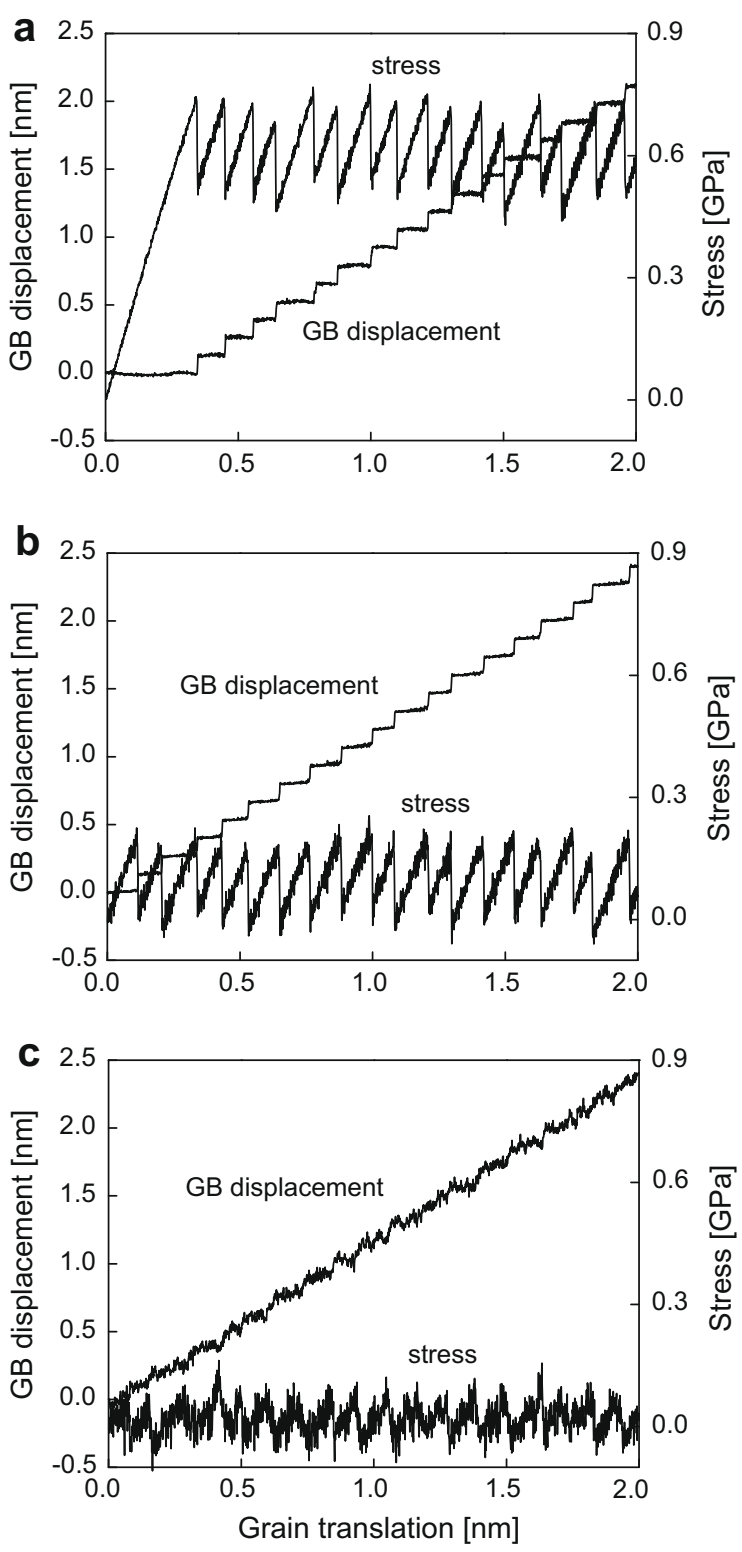

Fig. 9. GB displacement and shear stress at (a) $100 \mathrm{~K}$, (b) $500 \mathrm{~K}$ and (c) $900 \mathrm{~K}$ and the imposed grain translation velocity $1 \mathrm{~m} \mathrm{~s}^{-1}$ for the $\Sigma 21 \mathrm{~GB}$ in $\mathrm{Al}$ [370]. Note that the peak stress decreases with temperature. Above $900 \mathrm{~K}$, the stress behavior becomes noisy as the boundary begins to make occasional backward jumps. Through all these changes in the boundary dynamics, the coupling factor $\beta$ remains very close to its ideal (geometric) value.

confirmed by MD simulations [370]. There is an interesting similarity between the coupled GB motion in crystals and other cases of stick-slip dynamics existing in nature, e.g. the tip movements in atomic friction microscopy [402-405].

At high temperatures and/or slow velocities the GB can make occasional backward jumps, the stress behavior becomes noisy and the stick-slip dynamics eventually transform into driven random walk. In this regime, the role of the applied stress is to bias the rates of the forward and backward jumps, driving the GB predominantly forward [406]. This regime is characterized by a linear stress-veloc- ity relation known experimentally, $v=M \tau$; here, the $M$ is referred to as GB mobility (Section 9). During this dynamic transition from the stick-slip regime to driven Brownian motion, the GB remains perfectly coupled and follows a geometrical value of $\beta$.

A major challenge in the area of interface dynamics simulations is overcoming the huge gap between typical experimental GB velocities $\left(0.1-10 \mu \mathrm{m} \mathrm{s}^{-1}\right)$ and the extremely high velocities implemented in MD simulations (1-10 m $\mathrm{s}^{-1}$, Section 9). Due to the time-scale limitation of regular $\mathrm{MD}$, the lower bound of accessible velocities is currently on the order of $0.1-0.01 \mathrm{~m} \mathrm{~s}^{-1}$, depending on the model size. In the future, this gap could be closed by employing the accelerated MD methods [4-6,8,192,407]. Their promise for interface dynamics has been recently demonstrated by applying the parallel replica dynamics methods to reach GB velocities as low as $1 \mathrm{~mm} \mathrm{~s}^{-1}$ for coupled GB motion in copper [369].

\subsection{Interfaces and fracture}

On the atomistic level, mechanical energy dissipation occurs in two fundamental modes: shearing events, where atoms switch neighbors, and decohesion events, in which atoms lose the net number of neighbors - often irreversibly $[55,408]$. The former is a "renewable" dissipation mode since the newly switched neighbors can switch again, whereas the latter is non-renewable since the lost neighbors cannot be lost again. In Sections 6.1, 6.2 and 6.3 we mainly considered the former dissipation mode at interfaces; in this section we survey the latter. The two modes are of course coupled: shear-induced incompatibilities piled up at or trapped in the interface can trigger decohesion [193]. Vice versa, small changes in decohesion characteristics can induce large collateral changes in the shear dissipation [409].

Atomistic studies of intergranular fracture in elemental metals have a long history that will not be reviewed here $[12,215,410,411]$. While some simulations have been performed on polycrystalline samples [412-415], most of the physical insight comes from fracture studied of individual plane GBs with carefully chosen orientations of the applied load, crack front and lattice slip systems [411,416,417]. Such simulations have clarified the effects of the bicrystallography, generalized stacking fault (GSF) energies, temperature and other factors on the dislocation nucleation, twinning and other processes occurring at the GB crack tip. The dynamics of intergranular crack propagation and different mechanisms of energy dissipation have been studied in great detail $[418,419]$. As a promising recent development, an approach has been proposed to derive tractionseparation relations from MD simulations, providing atomistic-based input for cohesive-zone models for continuum fracture simulations [420-422].

The effect of impurities on GB fracture is another important direction that was briefly discussed in Section 5.3. Experimentally, it is known that small bulk concentrations 
of solutes can cause dramatic differences in the fracture mode of steels $[423,424]$ and intermetallics [425-429], where the material simply "falls apart" along interfaces to have clean intergranular fracture. Environmental effects such as hydrogen can also embrittle the interfaces [430,431]. Although we have come a long way towards experimental characterization of chemically complex interfaces under stress [281,432-436], atomistic calculations [437-439], especially by first-principles methods which can handle the complex interfacial chemistry, are invaluable for providing energetic information (Section 5.3).

At the mesoscopic level, well-known analytical models exist regarding both the quasi-static [440-442] and dynamic $[443,444]$ aspects of segregation-induced/delayed interfacial decohesion. As discussed in Section 5.3, the effects of solutes/impurities on mechanical decohesion can be roughly classified in two types $[437,438,445]$ :

- structural, such as atomic size differences [274],

- chemical contributions, such as electronegativity differences causing charge transfer and bond order change $[446,447]$, and/or other wavefunction- or magnetization-dependent features of the interfacial electronic structure [448-452].

Another way to look at a chemo-mechanical reaction $[197,198]$ such as interfacial decohesion is to consider its thermodynamic driving force [442] vs. kinetic aspects $[439,453]$. The thermodynamic aspect is solely concerned with the free energy change before and after the decohesion [442], with varying segregation, whereas the kinetic view is also concerned with the detailed pathway and the activation barrier [197,198], as well as with the solutes/impurities acting as catalysts or poisons $[430,453]$ for the chemomechanical reaction. Therefore, path-dependent information such as the maximum decohesion stress is also calculated [438,454-457].

Many kinds of interfacial decohesion have now been modeled with atomistic methods [438]: GBs of metals [274,445,448,446,457-463] and ceramics [464-467], metalceramic interfaces [437,468-470,466,471-474], hydrogen embrittlement $[475-478,469,479]$ and complex hetero-phase ceramic-ceramic interfaces [480] (even with intergranular glassy films [481]).

\section{Mechanical behavior on the nanoscale}

In Section 6 we surveyed the elementary mechanical responses of interfaces. In this section we focus more on the aggregate behavior. Nanostructured materials [482] have proliferated recently, in the form of bulk nanocrystals [483-486], thin films and multilayers [487-491], nanoporous materials [492-494], nanowires [495,496], nanopillars [201,497-500], nanopyramids [501], nanospheres [502,503], etc. Internal interfaces and/or free surfaces abound in these materials. While bulk dislocation activities are typically still indispensable for the materials low-temperature plasticity
[504], interfaces play ever-more-important roles regulating the dislocation dynamics, as well as directly contributing to the plastic strain, with shrinking size scale [505-515]. The characteristic sizescale $d$ can be the grain size, the layer thickness of the thin films or multilayers, the diameter of surface-confined nanowires, etc. A "Hall-Petch"-like strength vs. size correlation, $\sigma=\sigma_{0}+k d^{-\alpha}$, has been established experimentally for a great number of material systems, often with a "strong" power-law scaling exponent $\alpha$, such as $0.5[485,490,498,499,516,517]$. In nanostructured materials, this boundary contribution $k d^{-\alpha}$ to the total plastic flow resistance can dominate over the bulk plastic flow resistance $\sigma_{0}$. Then, at very small characteristic sizes, such as $d_{\mathrm{C}} \sim 20 \mathrm{~nm}$, a plateauing of the strong size scaling (Hall-Petch saturation) or even softening (inverse HallPetch) sets in with decreasing $d[485,506,508,512]$.

Atomistic simulations have provided significant knowledge about the interface- and surface-controlled plasticity, especially on the smaller end of the $d$-spectrum around the Hall-Petch saturation or inverse Hall-Petch transition [506-511,515]. Due to computational constraints, it is not feasible to directly simulate the larger- $d$ behavior with atomistic methods, and mesoscale techniques such as discrete dislocation dynamics (DDD) simulations are needed [190,513,514,518-522]. The two methods have offered an interesting contrast not only in the spatial, but also in the temporal behavior of dislocations at different $d$ 's. In DDD simulations of larger- $d$ micropillars [513,514,522], dislocation nucleation from surfaces was not implemented, nor did this seem necessary because, once mobile dislocations are seeded in the crystal as the initial condition, the sample is able to evolve and maintain a permanent population of mobile dislocations inside (DDD simulations use atomistic information about dislocation mobility [500,523], junction strength [190,520], cross-slip rate [524], etc., and the dislocation population can multiply and evolve inside the pillar with absorbing surfaces serving as the boundary condition). Thus, at any arbitrary time, it is possible to find one or more mobile dislocations inside the DDD simulated micropillars [513,514,522]. Strong power-law scaling is predicted from the DDD simulations, with the scaling exponent $\alpha$ close to the experiments [500,514,522].

In contrast, in most atomistic simulations of the smaller- $d$ nanocrystals, nanopillars and nanowires, one sees individual dislocations nucleate at the boundary, propagate across the crystal and get absorbed in other boundaries. At any arbitrary point of time during the atomistic simulation, one is likely to find some sessile dislocation structures or stacking faults stored in the crystal, but quite often no mobile dislocations. Thus, there appears to be a mechanism transition in the simulations at different $d \mathrm{~s}$, from the collective dynamics of an ensemble of mobile dislocations interacting with each other persistently within each grain and with boundaries, to individual mobile dislocations interacting transiently with boundaries at $d \sim d_{\mathrm{C}}$ (see Fig. 10a). 

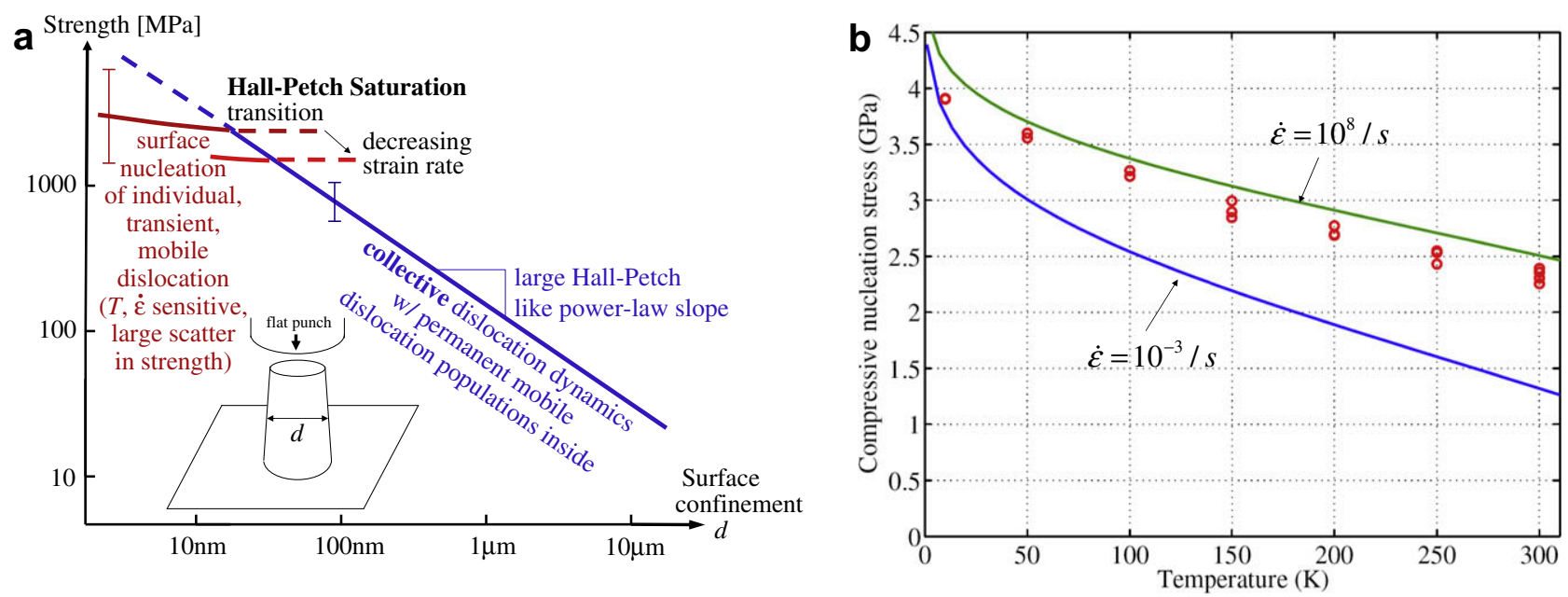

Fig. 10. (a) Predicted "Hall-Petch saturation" transition for surface-confined nanopillars, nanowires, nanospheres, etc. from atomistic calculation of surface dislocation nucleation [201]. The blue line illustrates strong power-law scaling of strength vs. size, due to surface-regulated collective dislocation dynamics: in this regime multiple dislocations interact and multiply via the Frank-Read mechanism, and the sample can support a permanent mobile dislocation density inside. The red curves illustrate transition to weaker size scaling: in this regime there are no permanent mobile dislocations inside, and in order to deform, new mobile dislocations must be nucleated afresh from surface sources, move transiently in a contiguous crystal volume and then disappear [499,504]. In addition to the transition from strong to weak size scaling, atomistic calculations also predicted enhanced temperature and strainrate sensitivities (see (b)), as well as a larger scatter in the measured flow stress, due to the smaller activation volume [201,504]. (b) Atomistically calculated surface dislocation nucleation stress in a $\mathrm{Cu}$ nanowire as a function of temperature and strain rate $\dot{\epsilon}$ from TST calculations (solid lines, $\dot{\epsilon}=10^{8} \mathrm{~s}^{-1}$ and $10^{-3} \mathrm{~s}^{-1}$ ) and direct MD simulations (circles, $\dot{\epsilon}=10^{8} \mathrm{~s}^{-1}$ only). The MD and TST calculations are independent of each other besides using the same interatomic potential [110]. Taken from Ref. [201] with permission. (For interpretation of the references to color in this figure legend, the reader is referred to the web version of this article.)

From the above discussion we see that it is important to distinguish between short-range boundary interactions due to direct atomistic kinetics occurring near interfaces or surfaces $[200,201,515]$, which likely extend no more than a few nanometers from the boundary, and the long-range boundary confinement effect on bulk dislocation dynamics $[521,525,526]$. The long-range effect could arise via dislocation free arms emanating from the boundary, the lengths of which are on the order of $10 \mathrm{~nm}$ or longer [514,522], and elastic interactions that can be even longer-ranged, as in the dislocation pile-up model $[516,517,527,528]$. Such a long-range boundary interaction effect is usually outside the realm of direct atomistic simulations.

Mughrabi et al. [529] measured the dislocation density in bulk work-hardened single-crystalline copper to be $\rho=3 \times 10^{14} \mathrm{~m}^{-2}$, corresponding to an average dislocation spacing of $s=\rho^{-1 / 2} \sim 50 \mathrm{~nm}$. Thus, if a contiguous crystal volume is smaller than $s^{3}$, there may not be a single dislocation in it. Because the existence or nonexistence of one mobile dislocation in a contiguous crystal volume, or "1to-0", is a "quantized" transition for the Frank-Read type of dislocation breeding mechanisms [530], this is yet another way to speculate that a transition in mechanism could occur when $d$ approaches tens of nanometers due to an extreme boundary confinement. Under such dislocation-starvation conditions [499,504], mobile dislocation nucleation from interfaces and/or surfaces becomes the strength-controlling mechanism (red curve in Fig. 10). Using the newly developed free-end NEB (FE-NEB) method, Zhu et al. [200] calculated the activation energies and activation volumes of surface dislocation nucleation from an atomically smooth surface, surface steps and kinks in $\mathrm{Cu}$, and found the activation volumes [193] to be in the range of $1-10 b^{3}$ [201], at the experimentally realistic strain rate of $\dot{\epsilon}=10^{-3} \mathrm{~s}^{-1}$. According to a TST analysis, such small activation volumes must lead to very sensitive temperature and strain-rate sensitivities of the flow stress. As shown in Fig. 10b, at $\dot{\epsilon}=10^{-3} \mathrm{~s}^{-1}$ FE-NEB/TST calculations predict a factor of three decrease in the average flow strength from 10 to $300 \mathrm{~K}$. Note that the FE-NEB/TST calculation has no adjustable parameters of its own, and besides using the same interatomic potential, is essentially independent of the MD simulations. To validate this new atomistic method, a comparison is made with direct MD simulations performed at $\dot{\epsilon}=10^{8} \mathrm{~s}^{-1}$ and various temperatures. The agreement between the two independent atomistic methods is excellent. We also see in Fig. 10b that going from the MD-accessible strain rate of $\dot{\epsilon}=10^{8} \mathrm{~s}^{-1}$ to the experimentally realistic strain rate of $\dot{\epsilon}=10^{-3} \mathrm{~s}^{-1}$ will give a factor of two decrease in the surface dislocation nucleation stress at $300 \mathrm{~K}$.

To better understand the short-ranged dislocation-GB reactions, it is often advantageous to regard GBs as "ad hoc slip systems". Such a view is supported by recent atomistic simulations of GB sliding and migration (Sections 6.2 and 6.3) for, just like lattice slip systems, GBs can also shear under applied stresses, possessing a nominal dislocation content given by the Frank-Bilby equation [367]. Thus, on a fundamental level, dislocation-GB reactions are no different from any other kinds of dislocation reac- 
tions. These reactions must satisfy the conservation of the Burgers vector, after the Frank-Bilby GB dislocation content is taken into account. GBs thus serve as reservoirs of dislocation content (lattice incompatibility), dynamically absorbing, desorbing and converting the dislocation content during deformation processes together with the lattice [200].

The ad hoc slip system view of GBs facilitates the understanding of phenomena such as the inverse Hall-Petch effect [506,508], since at very small grain sizes the operation of weak "ad hoc slip systems" will more than compensate for their confinement effect on the operation of normal lattice slip systems [512]. Formal similarities aside, there are important differences between ad hoc and normal slip systems. Normal lattice slip systems have a small multiplicity: in fcc metals there are only 12 well-defined lattice slip systems. In contrast, there are infinite possibilities of GB ad hoc slip systems, statistically distributed. Normal lattice slip systems have a high spatial density: every two adjacent atomic planes is a potential one. In contrast, the spatial density of ad hoc slip systems is inversely proportional to the grain size $d$. Even for a $10 \mathrm{~nm}$ grain size, the relative density of ad hoc slip systems is no more than a few percent. Nonetheless, that is sufficient to give rise to the inverse Hall-Petch behavior. This is because ad hoc slip systems are typically much weaker in shear compared to normal slip systems. The fundamental characterization of a slip system is the GSF energy [54,531-534], and the calculated GSF energies of ad hoc GB slip systems are typically smaller than for lattice slip systems [535] due to the larger structural disorder. This has profound consequences for the behavior of GB dislocations. With a weak GB GSF, the GB dislocation core tends to be more delocalized, with a smaller core energy. This tends to make clean (as- annealed) GBs strong traps for the lattice dislocation flux at the beginning of deformation (see Fig. 11).

For applications, ductility is sometimes as important as strength. The failure mode of nanostructured metals is often shear localization first [491,536-538], followed by micro-crack nucleation and growth. Thus, a strategy to delay catastrophic failure would be to (a) delay the onset of dramatic necking and (b) delay decohesion of interfaces. For (a), both linear (Consid'ere-Hart [539]) and nonlinear (Hutchinson-Neale [540]) continuum analyses point to the importance of the aggregate strain-rate sensitivity [200] and strain hardening rate in stabilizing near-uniform plastic flow. The essence of the Hall-Petch relation is that most of the plastic flow resistance may arise from interfacial resistance. Therefore interfacial hardening or the lack thereof, or even interfacial softening, may also govern the strain hardening of nanostructured materials to a large degree.

Atomic structures of interfaces can have a profound effect on their properties [541] (Section 6). Experimentally, there is evidence that the type of internal interfaces can have a strong effect on the aggregate ductility. Coherent twin boundaries are found to be superior to general GBs in preserving the aggregate ductility while still imparting high strength [542,543], presumably due to a larger hardenability during plastic flow [200] (issue (a) above), as well as better decohesion resistance (issue (b)).

Experimentally, crystalline-amorphous nanolaminates are also found to possess enhanced ductility [491]. It has been argued based on atomistic simulations that the amorphous-crystal interfaces (ACI) may be more damage-tolerant and enable better aggregate ductility than general GBs, due to their unique structures (only 2 inclination degrees of freedom, in contrast to general GBs,
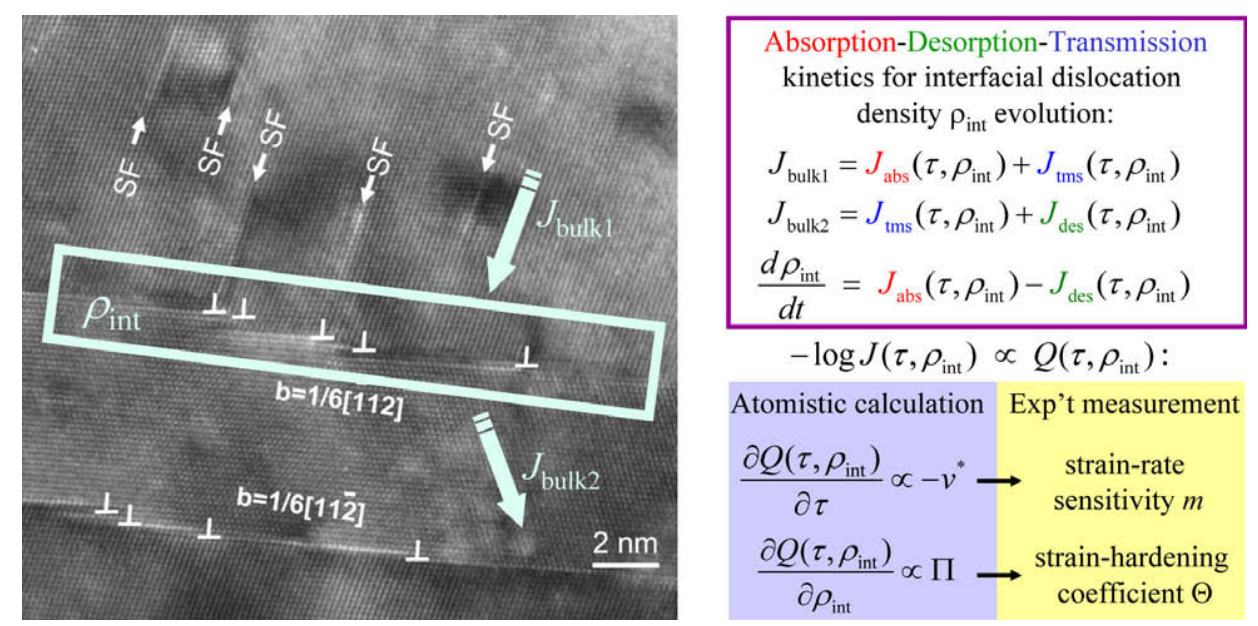

Fig. 11. Transmission electron microscopy image showing the accumulation of interfacial dislocation content $\rho_{\text {int }}$ inside a coherent twin boundary of nanotwinned $\mathrm{Cu}$ (from [638]), causing subsequent hardening of this interface. Time-averaging over discrete thermal activation events of dislocation absorption, desorption and transmission at the interface establishes an absorption-desorption-transmission kinetic equation, with $\rho_{\text {int }}$ as the internal state variable. Sensitivities (first-order derivatives) of the atomistically calculated activation energy $Q\left(\tau, \rho_{\text {int }}\right)$ with respect to the local stress $\tau$ and microstructural parameter $\rho_{\text {int }}$ can be related to the strain-rate sensitivity and strain hardening rate of the aggregate. Taken from Ref. [200] with permission. 
which have 5 degrees of freedom) and slip-transfer characteristics [491]. An ACI transducer's "ballistic shear" (dislocation slip) on the crystal side $\leftrightarrow$ "diffusive shears" on the amorphous side, which are carried by individual shear transformation zones [544-546] with infinite possibilities of Eshelby transformations [547], statistically distributed in the glass. As a result, while it is possible for an entire line of dislocation to get stuck in front of a $\mathrm{GB}$ as in the classic dislocation pile-up scenario of Hall and Petch [516,517], in front of an ACI it is possible to get stuck at points due to unfavorable kinetics, but it is much less likely for an entire line to get stuck [491].

\section{Grain boundaries at high temperatures}

\subsection{Grain boundary melting}

The atomic structure of surfaces, GBs and other interfaces tends to become increasingly disordered at high temperatures, especially when approaching the bulk melting point $T_{m}$. The thermal disordering of GBs can affect their sliding resistance [548,549], diffusion [550-552] and Coble creep rates, as well as their ability to move under applied shear stresses [368] or other driving forces, emit and absorb dislocations, and act as sinks and sources of vacancies. It has long been supposed that at high temperatures, surfaces and interfaces can develop a liquid-like layer, which grows wider as the temperature approaches $T_{m}[12,553,554]$. Despite the experimental efforts [548,555-561] and computer modeling by diffuse-interface [562-566], phase-field crystal $[567,568]$ and atomistic [549-552,569-576] methods, the thermodynamic nature of the GB premelting transition is not well established.

In recent $\mathrm{MD}$ simulations [550], high-angle $\mathrm{GBs}$ in $\mathrm{Cu}$ were observed to accumulate significant structural disorder with increasing temperature and eventually form a liquidlike layer about $10 \mathrm{~K}$ below $T_{m}$. In this premelting temperature range, the local structure factor in the GBs tended to zero, whereas the GB diffusion rapidly accelerated and approached the diffusivity of the bulk liquid phase at $T \rightarrow T_{m}$ (Section 8.2). In a more recent study [292], the liquid-layer thickness in the $\Sigma 5(210)\left[\begin{array}{ll}0 & 01\end{array}\right] \mathrm{Cu} \mathrm{GB}$ was found to rapidly increase with temperature near $T_{m}$ but remain finite at $T_{m}$. This boundary could be slightly overheated above $T_{m}$, indicating that its melting is a first-order transition. In recent MD simulations of $(001)$ twist GBs in $\mathrm{Si}$ [577], the high-angle boundaries $\left(37^{\circ}\right.$ and $45^{\circ}$ misorientation) displayed a continuous disordering transition with an apparently diverging width. A complete structural disorder of these boundaries was reached only at $T_{m}$. At temperatures below $T_{m}$, the boundaries retained at least some degree of structural order, with local fluctuations between the ordered (with recognizable structural units) and disordered states with a frequency increasing near $T_{m}$. At the same time, a low-angle $\left(16^{\circ}\right)$ boundary displayed a higher degree of structural order at all temperatures up to $T_{m}$ and retained a finite width at $T_{m}$.
One can expect a certain parallel between premelting phenomena at GBs and at open surfaces. The (111) surface of $\mathrm{Cu}$ is very stable and shows no signs of premelting, remaining metastable over a relatively wide $(30-40 \mathrm{~K})$ temperature range above $T_{m}$ [578]. By contrast, the (110) $\mathrm{Cu}$ surface melts continuously and displays a divergence of its thickness to infinity as temperature approaches $T_{m}$ [335]. The high stability of (111) fcc surfaces against premelting was also confirmed for $\mathrm{Al}$ [579] and $\mathrm{Au}$ [580]. The situation in bcc metals is different. The (1 111 ) surface of bcc $\mathrm{Nb}$ and $\mathrm{V}$ premelts continuously, with the liquid-layer thickness diverging as $-\ln \left(T_{m}-T\right)$ as $T \rightarrow T_{m}$. By contrast, the (100) and (1 10$)$ $\mathrm{V}$ surfaces remain ordered up to $T_{m}[581,582]$. These effects demonstrate the rich nature and complexity of interface premelting behaviors, which can range from a continuous process to a first-order transition, depending on the interface type and crystallographic orientation. While known and well studied for surfaces [579,583], this rich complexity is only beginning to be explored for GBs.

Attempts to understand the different scenarios of GB premelting often involve the concept of disjoining potential $\Psi(h)$, which represents the interaction free energy between the two solid-liquid interfaces bounding the liquid GB film as a function of their separation $h$. Theoretical models $[584,585]$ can predict the entire premelting behavior knowing only the disjoining potential and the thermodynamic functions of the bulk phases. For example, a purely repulsive disjoining potential would lead to a continuous premelting, whereas a combination of longrange attraction and short-range repulsion results in a finite $\mathrm{GB}$ width at $T_{m}$. While various physical factors influencing the disjoining potential have been discussed $[554,586]$, no theory unambiguously predicts $\Psi(h)$ for metallic GBs. Two-dimensional phase-field crystal simulations [567,568] demonstrate that some GBs feature a repulsive $\Psi(h)$ while others a combination of repulsion and attraction. Hoyt et al. [587] have recently proposed a method for extracting $\Psi(h)$ from MD simulations by analyzing width fluctuations of a premelted GB. Their method has been applied to a high-energy $\Sigma 5$ twist GB in $\mathrm{Ni}$ at temperatures up to $2 \mathrm{~K}$ below $T_{m}$, demonstrating that the disjoining potential is exponentially repulsive. Further research is needed to determine if GBs with lower energies can exhibit an attractive component of $\Psi(h)$.

Most of the previous simulation work was focused on premelting behavior of elemental crystals. GB premelting in alloys has recently been studied by semi-grand-canonical MC simulations employing the $\Sigma 5(210)[001]$ GB in $\mathrm{Cu}-$ rich $\mathrm{Cu}-\mathrm{Ag}$ alloys as a model [292]. Ag atoms strongly segregate to this boundary and produce significant distortions of its structure due to the large atomic size mismatch between $\mathrm{Cu}$ and $\mathrm{Ag}$. The $\mathrm{GB}$ disorder rapidly increases as the bulk composition approaches the solidus line from below. At the same time, the local chemical composition inside the boundary approaches the liquidus composition at the respective temperature. This behavior clearly indicates the formation of a liquid-alloy layer in the GB region 
when the bulk composition and temperature approach the solidus line. Among other interesting findings, the thickness of the liquid layer remains finite at the solidus line and the GB can be overheated and/or oversaturated to metastable states slightly above the solidus. Together with the continuous behavior of the local chemical composition and local structure factor in the GB region when crossing the solidus line, these observations point to the first order of the premelting transition in alloys. It remains to be seen whether this behavior is generic or specific to this particular boundary.

\subsection{Grain boundary diffusion}

Because the diffusivity of atoms in GBs is orders of magnitude faster than in the lattice, GB diffusion can control many processes in materials, including microstructure development, many phase transformations, creep, and some modes of plastic deformation and fracture [588]. Although a large volume of experimental data has been accumulated over the recent years [588-590], many aspects of GB diffusion remain poorly understood, including the diffusion mechanisms on the atomic level. Significant progress has been achieved through atomistic computer simulations [550,591-598], which revealed a number of new GB diffusion mechanisms that are profoundly different from known mechanisms of lattice diffusion.

To investigate the diffusion mechanisms, a single point defect (vacancy or self-interstitial) is created at various positions in the GB core and its formation free energy is evaluated using molecular statics and the harmonic approximation to atomic vibrations. From the defect-free energies, the equilibrium defect concentrations at different sites are computed and the most abundant (and thus most important for diffusion) defects are identified [550,595,596]. A single defect is then allowed to walk along the GB by running MD simulations, and the MD snapshots and atomic trajectories are analyzed to determine the most typical diffusive events induced by the defect. Simulations show that such events, or transitions, often represent complex atomic rearrangements involving a collective displacement of several atoms. The absolute rates of the transitions are computed using the harmonic TST [189] with the saddle-point search implemented by the NEB method.

Two different approaches have been developed to calculate the diffusion coefficients. In the first approach, the defect jump rates are compiled into a rate catalog, which is fed into KMC simulations [595,596]. Also knowing the defect concentrations, the KMC simulations permit accurate calculations of the diffusion coefficients in various directions in the GB core. Furthermore, by repeating the calculations for vacancies and interstitials separately, their contributions to GB diffusion can be compared in order to identify the dominant defect. However, the KMC method is only suitable at low temperatures, at which the boundary structure remains well ordered and diffusion is mediated by the motion of single point defects.
In the second approach, the diffusion coefficients are extracted directly from MD simulations [550-552,591, 598]. After bringing the boundary to point-defect equilibrium, an MD run is performed in which mean-squared displacements of atoms within the GB core are computed. The diffusion coefficient is extracted from the Einstein relation for various directions in the GB plane. Such calculations do not rely on the model assumptions inherent in the KMC simulations, but in order to accumulate adequate statistics of atomic jumps, the MD simulations are implemented at relatively high temperatures (typically, above $\left.0.6-0.7 T_{m}\right)$.

In recent years, both approaches have been applied to GBs in $\mathrm{Cu}, \mathrm{Ag}$, Ni and $\mathrm{Al}$, with different $\Sigma$ values and different orientations of the tilt axis [550,552,591-597] or twist axis $[598,599]$. The simulations have revealed a number of generic properties of point defects and diffusion processes in GBs, which can be summarized as follows.

The vacancy formation energy in GBs is on average lower than in the bulk but can display very strong siteto-site variations, ranging from $10 \%$ of the bulk value to above the bulk value. The same is true about GB self-interstitials, whose formation energy is also on average lower than in the lattice and shows very strong site variations. The variability of the point-defect energies can be linked to the existence of alternating tension and compression regions in the GB core [596,597]. The interaction of point defects with GBs is short-ranged and is usually limited to about 2-3 lattice spacings around the core. Some interesting correlations have been found between the point-defect formation energies and the GB energy [596,597,600]. An important observation is that the average vacancy and interstitial formation energies in GBs are close to each other, suggesting that both defects can be equally important for GB diffusion.

GB vacancies and interstitials can exist in a variety of structural forms. Both defects can be localized at certain sites or delocalized over an extended area. Not all GB sites can support a stable vacancy. Often, when an atom is removed from a site 1 to create a vacancy and the structure is relaxed, the vacant site is filled by a neighboring atom 2 during the relaxation. As a result, the vacancy ends up at site 2 and not at the intended site 1. Surprisingly, delocalized and unstable vacancies are very common forms of GB defects, especially in high-energy boundaries.

The multiplicity of structural forms of point defects in GBs leads to a large variety of their diffusion mechanisms. Vacancies can move by simple exchanges with individual atoms, as they do in the lattice, but can also induce collective jumps of two to three atoms at a time [596]. Such collective jumps can be linked to the existence of unstable vacancies and have been found in many boundaries. Interstitial atoms can migrate by hopping between interstitial positions, or can move by indirect jumps involving a collective displacement of several atoms. In such collective jumps, an interstitial atom kicks out a neighboring regular atom into an interstitial position in a neighboring struc- 
tural unit and takes its place. The displaced atom, in turn, can kick out its neighbor and the latter becomes an interstitial atom, etc. Interstitial jumps involving up to four atoms moving in a concerted manner have been found in some GBs. Point defects can also induce ring processes, in which a group of atoms implements a collective displacement in a cyclic manner [595]. Some of such ring mechanisms can happen spontaneously, without any preexisting defects. Overall, a prototypical diffusive event in GBs is a collective displacement of an atomic chain (string) in which the head atom fills a relatively open space (free volume) while the trailing atom leaves a similar open space behind [550,601]. There is an intriguing similarity between diffusion mechanisms in GBs and diffusion in supercooled liquids [602] and metallic glasses [603].

KMC simulations at low temperatures lead to the conclusion that there is no unique mechanism of GB diffusion: either vacancies or interstitials can dominate the diffusion flux, depending on the GB structure, the temperature and even the diffusion direction. In spite of the involvement of multiple diffusive events with different activation energies, the GB diffusion coefficients still follow the Arrhenius law with reasonable accuracy, suggesting that the diffusion process is controlled by one type of jump or perhaps a group of jumps with nearby activation energies. The anisotropy of GB diffusion can be very significant, especially at low temperatures. In high-angle tilt GBs, diffusion along the tilt axis is not necessarily faster than diffusion normal to it, contrary to the common assumption. At a given temperature, the diffusion coefficients can vary by several orders of magnitude, depending on the GB structure. Given these huge variations, the frequently used notion of an "average" GB diffusivity in a polycrystalline material may not be a well-defined physical quantity.

The MD simulations reveal positive deviations of GB diffusion from the Arrhenius law at high temperatures, where the diffusion coefficients rapidly increase and continuously approach the diffusivity of the bulk liquid phase as the temperature approaches $T_{m}$. At these temperatures, the diffusion coefficients in different GBs tend to converge, or even merge, suggesting a transition to a liquid-like mechanism of diffusion [550,551]. Importantly, such mergers can occur well below the premelting temperature range when the GB structures are still relatively ordered. This finding indicates that the local liquid-like structures responsible for the diffusion process are not static but constantly form and disappear in a manner similar to heterophase fluctuations between the solid and liquid phases $[553,604,605]$. A quantitative theory of such fluctuations and their effect on GB diffusion could be the subject of future research.

The calculated GB diffusion coefficients in $\mathrm{Cu}[337,550]$ are in reasonable quantitative agreement with experimental measurements [606]. A similarly encouraging agreement has recently been found for dislocation diffusion in $\mathrm{Al}$ [607,608]. This agreement demonstrates that atomistic simulations become capable of predicting quantitatively correct short- circuit diffusivities in metals, on top of providing insights into the underlying diffusion mechanisms. The latter information is extremely difficult to obtain experimentally.

\section{Interface motion}

The development and evolution of materials microstructure is in many cases influenced by the motion of interfaces, including GBs and heterophase interfaces. For solid materials, recrystallization, grain growth and certain phase transformations involve migration of GBs under various driving forces. In rapid solidification, the non-equilibrium properties of solid-liquid interfaces, namely their mobility and velocity-dependent partitioning coefficients, are known to play important roles in governing growth morphologies, solute partitioning and phase selection. The rates and microstructural morphologies associated with many solidstate transformations are strongly influenced by interface mobilities and the associated non-equilibrium solute partitioning across the interfaces.

In this section we review atomistic methods for studying interface motion, focusing specifically on GB migration. The techniques discussed below share many features in common with those applied in studies of solid-liquid interface kinetics; the interested reader is referred to very recent reviews [325,328] of the application of atomistic simulations in this context. To date, much less work has been done for solid-solid heterophase interfaces, although it is expected that some of the methods discussed below should be applicable to such interfaces as well.

The driving forces for GB migration typically originate from either capillary effects (e.g. during grain growth) or a difference between strain energy densities on either side of the boundary (e.g. during recrystallization). In some materials, GBs can be moved by an applied magnetic field [609-611] or other physical forces. Much of the current empirical knowledge about GB migration comes from experiments on bicrystals, in which individual boundaries with precisely characterized bicrystallography are moved by capillary forces $[389,612]$. Atomistic simulations have been extensively applied in this field, despite the challenges imposed by the time and length-scale limitations of the MD method. Although the simulated GB mobilities tend to be much higher than the experimental ones (see below), reasonable qualitative agreement with experiment has been demonstrated in a number of cases, and a glimpse of atomic mechanisms of GB migration is beginning to emerge [598,601].

A number of simulation schemes have been developed for GB migration studies, some of which will be briefly discussed below.

\subsection{Curvature-driven motion}

In this method, the GB is curved and moves by capillary forces. Although curvature driven GB migration has been observed in nanocrystalline samples containing several 
grains [377,613], most simulations are performed on bicrystals with crystallographically controlled GBs. Such simulations are thus qualitatively similar to the bicrystal experiments. For example, the half-loop geometry employed in the experiments [612] has been implemented in the computer, first in 2-D simulations with a LennardJones potential $[614,615]$ and more recently in 3-D simulations with an EAM Al potential for $\langle 111\rangle$ tilt GBs [616]. The capillary driving force $P$ is given by $P=\Gamma \kappa$, where $\kappa$ is the local mean curvature and $\Gamma=\gamma+\gamma / \prime$ is the GB stiffness (Section 5). Since the boundary curvatures implemented in simulations are much greater than in experiments, the simulated driving forces are much higher than those realized in experimental measurements.

It is usually assumed that the local GB velocity $v$ is proportional to the driving force and the GB mobility $M$,

$v=M P$,

a relation which usually holds in experiments $[389,612]$. Thus, the measured or computed relation between $v$ and $\kappa$ yields the so-called reduced mobility $M^{*}=M\left(\gamma+\gamma^{\prime \prime}\right)$. The mobility $M$ itself remains unknown, unless the GB stiffness is determined in separate calculations, which is a difficult task (Section 5). Also, since the GB is curved and spans a range of different inclinations, the results represent an average mobility over a range of inclination angles. This creates an uncertainly affecting the studies of relations between the GB mobility and the bicrystallography.

\subsection{Strain-driven motion}

In strain-driven simulations [598,617-619], a constant elastic strain is applied to both grains of a bicrystal containing a plane boundary. The driving force is the difference between the elastic strain energy densities in the grains, arising due to elastic anisotropy of the crystal lattice. Thus, the method works best for crystals with a strong elastic anisotropy. The applied strain (which is usually biaxial) must be as high as a few percent in order to create a large enough driving force that would produce significant GB displacements during typical MD runs (1-10 ns). This method imposes certain restrictions on the bicrystallography, in order to prevent shear stresses parallel to the boundary plane and avoid symmetries that lead to equal strain energy densities in the grains. For example, the method is not applicable to symmetrical tilt GBs. However, by contrast to the previous method, the GB remains planar and the inclination angles remain fixed during the simulation.

Another advantage of the method is that the GB mobility can be extracted directly from the velocity-force relation without involving the GB stiffness. However, the driving forces created in strain-driven simulations tend to be higher than in the capillary method. While the linear dynamics (Eq. (7)) are still followed in many cases, some simulations display strongly nonlinear velocity-force relations [618]. (In such cases, it has been proposed to deter- mine the mobility by extrapolation to the small-velocity limit: $M=(d v / d P)_{v \rightarrow 0}[618]$.) It should be pointed out that under the extremely high driving forces and fast GB speeds $\left(>1 \mathrm{~m} \mathrm{~s}^{-1}\right)$ implemented in such simulations, the GB can be taken quite far away from equilibrium, which might be one of the causes of the nonlinear effects.

\subsection{Artificial driving force}

A driving force can be created by artificially altering the potential energy of the atoms, depending on the local lattice orientation [620]. This artificial potential can be set up to give one grain an energetic preference over the other, creating an energy density difference across the boundary that drives its motion. This driving force, which can be thought of as similar to the magnetic force [621], is easier to control in simulations than the elastic strain energy. This method offers the advantage that it should work for both plane and curved boundaries and does not require that the material be elastically anisotropic or that the boundary be asymmetrical. In addition, artificially driven simulations can be implemented in smaller systems and afford shorter MD times [620]. The method has been applied to study the mobility of twist and mixed boundaries in Al over a wide angular range of crystallographic orientations [620]. It has also been applied to symmetrical and asymmetrical $\Sigma 5$ tilt boundaries in $\mathrm{Ni}[621]$.

This method has one caveat, however: it relies upon the assumption that the GB mobility does not depend on the nature of the driving force, which remains an open question. Nevertheless, the recent comparison of artificial and elastically driven simulations of the same Ni GBs gave close values of the mobility in the limit of small forces [621].

\subsection{Random walk method}

Trautt et al. [622] proposed a method in which $M$ is extracted from MD simulations of a random walk of a planar GB subject to fully periodic boundary conditions. The walk is induced by thermal fluctuations and is expected to follow the kinetic laws of 1-D diffusion. The GB mobility can be determined from the "diffusion coefficient" of the GB treated as a particle implementing a 1-D random walk. Since no driving force is applied to the boundary, the results represent the true zero-force limit of the GB mobility. Simulations have been performed for several metals (Al, $\mathrm{Cu}, \mathrm{Ni}, \mathrm{Ag}, \mathrm{Pb}$ and $\mathrm{Au}$ ) modeled with EAM and Lennard-Jones potentials [622]. Where comparison could be made, the mobilities determined by this method were in agreement with values obtained by the capillary-force method (although a comparison of planar and curved boundaries should be taken with a grain of salt) but exceed the mobilities predicted by strain-driven simulations. The latter appears to suggest that GB mobility may depend on the nature of the driving force. The authors indicate that their method can be properly modified to account 
for the presence of impurities and can be applied for studying the solute-drag effect on the atomic scale [622].

\subsection{Capillary wave method}

This approach [259] is based on the analysis of spontaneous shape fluctuations of an initially plane GB that becomes rough at high temperatures (Section 5). The GB stiffness is extracted from spectral analysis of shape fluctuation amplitudes of the boundary in the long-wave limit. At the same time, by examining the decay of the correlation function of the fluctuations, the decay exponent can be extracted and combined with the stiffness to compute the GB mobility $M$. Thus, the stiffness and mobility are determined separately for exactly the same boundary. As in the random-walk method, the mobility obtained represents the zero-force limit.

This appears to be the only simulation method at present that gives access to the GB stiffness $\gamma+\gamma^{\prime \prime}$. Calculations of $\gamma$ at finite temperatures involve thermodynamic integration schemes (Section 5) and present a challenging task. Direct calculations of $\gamma^{\prime \prime}$ (i.e. from $\gamma$ as a function of inclination angle) would be even more challenging and have probably never been done. Knowledge of $\gamma^{\prime \prime}$ is important for the understanding of many simulation results and for interpretation of experiments [623].

The method is computationally intensive and works at relatively high temperatures when the GB is rough. At low temperatures (below the roughening transition), the boundary is atomically flat and analyzing its capillary waves is impossible. Moreover, some high-symmetry boundaries that produce cusps on the inclination-angle dependence of their energy tend to remain atomically flat until rather high temperatures, putting them effectively beyond the reach of this method. Simulations of the asymmetrical $\Sigma 7\langle 111\rangle$ tilt $\mathrm{GB}$ in $\mathrm{Ni}[259]$ indicate that the contribution of the $\gamma^{\prime \prime}$ term to the GB stiffness is significant and strongly anisotropic (i.e. it depends on the orientation of the rotation axis; see Fig. 4). The obtained GB mobilities have the same order of magnitude as predicted by other methods.

\subsection{GB motion during recrystallization}

Although simulations of curvature-driven GBs are interesting in connection with grain growth, they hardly represent GB motion during recrystallization. The straindriven and artificial-force simulations are more relevant, but the excess energy of the receding grain is distributed uniformly over its lattice, and the boundary interactions with lattice dislocations are not considered. It is only recently that $\mathrm{MD}$ simulations have been extended to GB motion driven directly by a dislocation density difference, in which the boundary encounters lattice dislocations distributed in the receding grain, Ref. [624,625]. To achieve this, dislocation arrays (low-angle boundaries) are inserted in one grain of a bicrystal, whereas the other grain remains dislocation-free. As expected, upon heating the high-angle boundary moves into the dislocated grain (Fig. 12), closely reproducing GB motion during a recrystallization process. The elimination of the lattice dislocations as the boundary consumes the deformed grain reduces the stored energy and provides the driving force for this process.

The initial study [624] employed a Lennard-Jones potential and only one type of dislocation. The approach was later extended to $\mathrm{Cu}$ and $\mathrm{Al}$ simulations with EAMtype potentials and to different types of moving GBs (tilt or twist) and dislocations (edge or screw) arranged in arrays with different densities and misorientation angles $[624,625]$. The GB was found to move by small increments corresponding to individual dislocation absorption events. For edge dislocations parallel to the GB, two types of absorption events were found:

- At low dislocation densities, the boundary cusps out towards the nearest dislocation and quickly absorbs it, followed by a relatively slow curvature-driven motion of the rest of the boundary that eventually eliminates the cusp.

- At high dislocation densities, the dislocation advances towards the GB and gets absorbed.

The actual GB motion is often a mixture of the two mechanisms. For screw dislocations, the absorption process is nearly continuous and the GB remains fairly flat, making the GB motion less irregular.

This important work demonstrates the feasibility of direct MD simulations of strain-induced GB motion into a deformed grain. Further applications of this approach could give insights into recrystallization mechanisms and dynamics. Interestingly, the migration velocity was found to be proportional to the estimated driving force $P$ in some cases but to $P^{2}$ in other cases [625], a nonlinear effect that calls for an explanation. The authors $[624,625]$ point to variations in the dislocation structures and density as an important factor in recrystallization dynamics. The GB mobilities obtained by this method are lower than in all other MD simulations but still higher than in experiments.

\subsection{Summary of results}

The simulations performed so far confirm that GB mobility depends on the GB crystallography, the temperature and the impurity content in the material. The computed mobilities and their activation energies examined as functions of crystallographic angles exhibit maxima or minima at or near special low- $\Sigma$ misorientations [598, 614-620], in agreement with bicrystal experiments. It should be noted that the temperature dependence of GB mobility can be highly nontrivial. For example, recent MD simulations of [001] tilt GBs in $\mathrm{Ni}$ [621] reveal a significant increase of $M$ above the roughening transition temperature. The transition temperatures and $M$ values were found to be very different for symmetrical and asymmetrical boundaries. Furthermore, even the character of the GB 
a

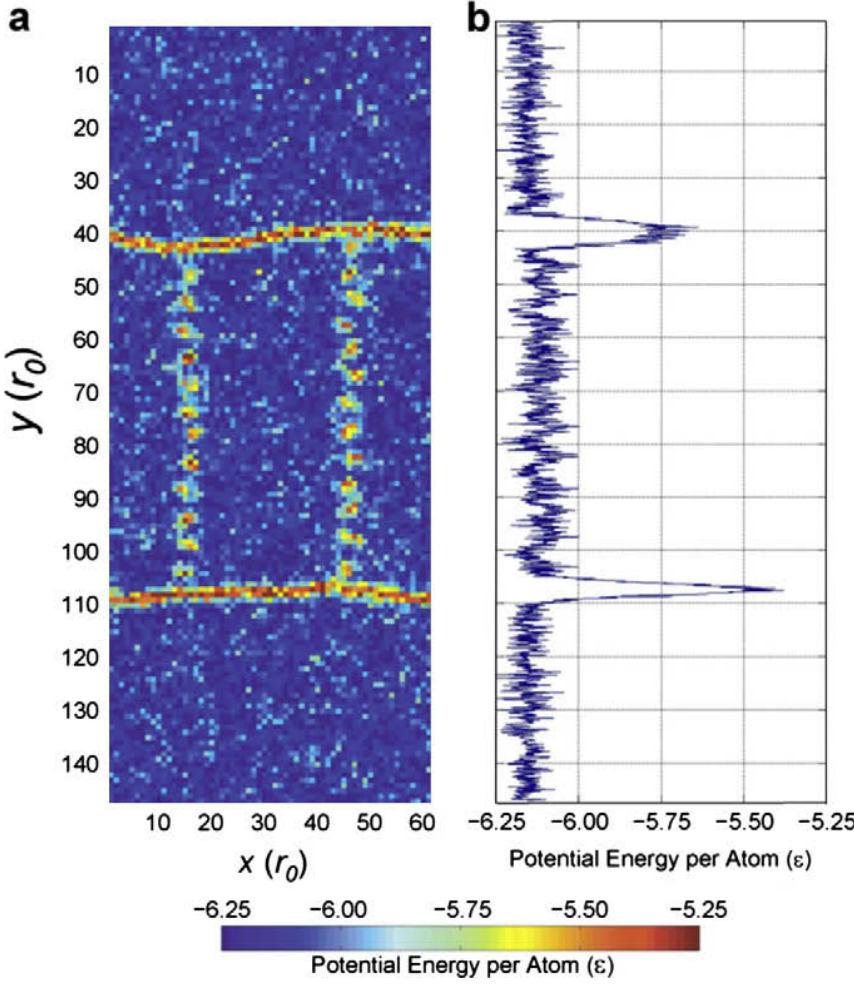

C

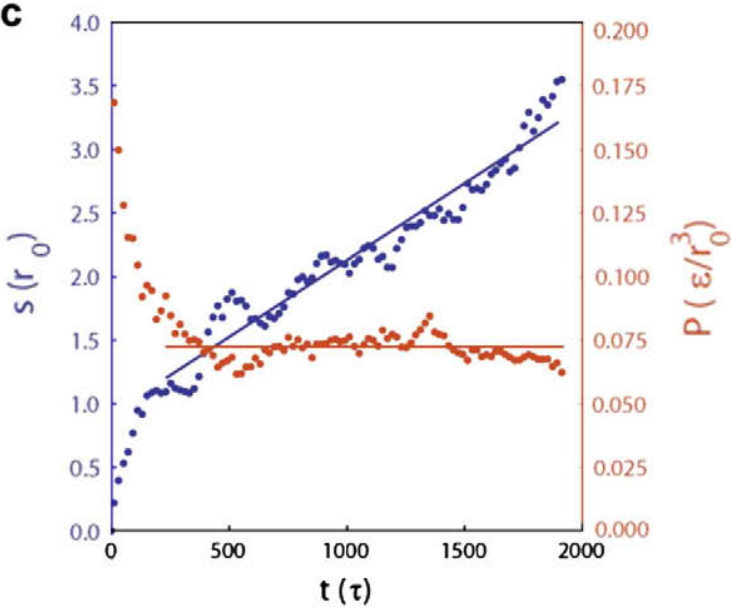

Fig. 12. MD simulation of GB motion driven by dislocation walls, mimicking GB migration during a recrystallization process [624]. (a) Top view of the simulation cell with atoms colored by potential energy. The two GBs are connected by two walls of edge dislocations. (b) The peaks of potential energy mark the GB positions and are used for their tracking. The GB displacement and driving force as functions of the simulation time. The GB velocity is obtained form the linear fit (shown) when the driving force becomes nearly constant.

motion changes from incremental below the roughening transition to continuous above it.

When GBs are moved by applied driving forces, such forces and the corresponding GB velocities greatly exceed the experimental values, a factor which should be taken into account when comparing simulations with experiments. The GB mobilities extracted from the simulations, no matter which method was applied, are consistently higher than experimental mobilities. Furthermore, the activation energies of GB migration always underestimate the experimental values, sometimes by as much as an order of magnitude.

The latter discrepancy is commonly attributed to the effect of impurities [626]. Even if the bulk impurity concentration is very small, its GB concentration can be high due to GB segregation. This explains the experimental fact that even extremely small amounts of certain impurities can produce very strong impacts on GB mobility [612]. Whereas all experiments are inevitably influenced by the impurity drag effect to one degree or another, the atomistic simulations are free from impurities. Optimistically speaking, this means that the simulations offer a unique opportunity to probe the intrinsic (impurity-free) mobility of GBs, which is important both from the fundamental point of view and as a reference point for a proper evaluation of the solute effect. However, the next step should be to include the solute drag effect into atomistic simulations.
The status and existing challenges in the solute drag area have been recently reviewed by Mendelev and Srolovitz [626]. Regular MD simulations remain impractical because of the time-scale limitation of the method. The solute drag involves diffusion of impurity atoms across the GB region and through the lattice, processes which are too slow to simulate on todays computers. Alternate approaches have been developed, such as Ising-type models implemented by KMC simulations. Although such simulations overcome the time-scale problem and advance the system along the migration path fast enough to capture some basic features, they rely on strong assumptions regarding the boundary structure and migration mechanisms. Nevertheless, such methods already offer a means of comparing simulation results with experiments, and for a critical evaluation of existing analytical models of the solute-drag effect.

\section{Outlook}

Atomistic simulations is a flourishing area of materials research, full of success stories, breakthroughs and unsolved problems. Some of the existing challenges and promising directions have been outlined in the relevant sections of this paper. We would like to conclude the paper by discussing a few more challenges and emerging directions of future research. 
The coming years may see new developments in the area of interatomic potentials. Continuous progress in this field is critical for expanding the atomistic simulations to new classes of materials and for improving their predictive capabilities. Potentials are quickly becoming a means of parameterization of first-principles databases, a transformation that happened to the cluster-expansion methods over a decade ago (Section 2.2).

It is important to continue the pursuit of new potential forms, including further extensions of EAM- and FS-type formalisms to systems with partially covalent bonding, charge transfer and magnetic energy. For example, encouraging progress has been reached in the development and application of bond-order potentials. In recent years, numerical BOPs have been successfully applied by Vitek and co-workers to study dislocations and GBs in transition metals and compounds [627]. Despite substantial advantages over EAM and FS potentials for transition metals, the numerical complexity prevents BOPs from application to large-scale MD simulations. The recently proposed analytical BOPs $[162,628,629]$ rely on analytical representations of energy and forces and might become suitable for largescale simulations. They generalize the second-moment approximation of electronic density of states underlying the FS formalism by including higher-order moments up to the sixth, and have been demonstrated to correctly reproduce structural trends and magnetic properties of transition metals. Some of the ideas developed in this and other areas, such as molecular simulations of chemical reactions, polymers and biological molecules, might be adaptable, or at least may serve as an inspiration, for new potential forms for metallic systems.

The development of more efficient potential fitting and testing procedures based on more rigorous statistical methods and new numerical algorithms can drastically improve the reliability of future potentials, eventually transforming the potential generation industry from art to science. There are outstanding issues even in the area of traditional EAMtype potentials. For example, a regular procedure should be developed for fitting ternary and higher-order systems (see the discussion at the end of Section 3.3).

The improved quality of potentials opens possibilities for more accurate thermodynamic calculations for solid and liquid phases using hybrid approaches combining classical MD and MC simulations with first-principles total energy calculations. One of the recently proposed approaches [630,631] permits free energy calculations on a DFT level of accuracy within a thermodynamic perturbation theory framework. The method starts out with free energies computed by thermodynamic integration using a classical interatomic potential, and then "corrects" them using first-principles calculations for only some of the reference configurations. If the reference configurations have been generated with an accurate potential, only a modest number of first-principles calculations is needed for convergence. The method has been demonstrated to work for concentrated solid and liquid alloys in binary systems [630].
This and similar approaches could eventually raise the accuracy of thermodynamic calculations to the DFT level (Section 2.2), and could be used for more accurate computations of phase diagrams, particularly in combination with the CALPHAD method. They could also provide reliable input data for phase-field simulations of microstructure evolution in alloys.

An excellent example of the impact that atomistic methods can produce on microstructure evolution modeling is given by the recent on-lattice KMC modeling studies of concentrated multicomponent systems, which was applied to investigate early stages of $\gamma /$-phase precipitation in $\mathrm{Ni}-$ Al- $\mathrm{Cr}$ alloys [632,633]. The model parameters, such as the pair-interaction and vacancy binding energies, vacancy jump barriers and attempt frequencies, were fit to a mix of first-principles and experimental data to achieve good agreement with known thermodynamic and kinetic properties of this system. The simulations have demonstrated a striking agreement with experimental 3-D atom-probe observations of precipitation kinetics, phase morphology and composition profiles across $\gamma / \gamma /$ interfaces, providing significant new insights into the origin of certain morphological features and into the role of diffusion kinetic factors [633]. The validity of this lattice model is justified by the small lattice misfit in this particular system. However, future extensions of this approach to more general cases of diffusion-controlled microstructure evolution would require the incorporation of elastic strain effects and other factors. Wang et al. [634] proposed accounting for longrange elastic effects by integrating a first-principles $\mathrm{CE}$ with KMC simulations, and applied this scheme in the context of morphological evolution of Guinier-Preston zones in $\mathrm{Al}-\mathrm{Cu}$ solutions [634]. In an alternative approach proposed recently by Rudd et al. [9], KMC simulations are combined with on-the-fly solution of the elasticity problem using the Lanczos recursion algorithm.

In the area of solid-solid interfaces, atomistic simulations have been actively applied to study the interface structure and equilibrium thermodynamic properties (Section 5.4) but not the kinetics of interface motion. For example, the motion of semicoherent and incoherent heterophase interfaces during diffusionless (martensitic) transformations can be readily accessible by regular MD simulations, yet such studies remain scarce $[635,636]$. Systematic atomistic investigations of the kinetics of interface migration during martensitic transformations, e.g. in a manner similar to the studies of GB migration (Section 9), could provide very useful input to mesoscopic phase-transformation models. The key ingredients for such studies are interatomic potentials predicting the correct structural transformations in the systems of interest. A recent example is given by the $\mathrm{Zr}$ potential reproducing the experimentally known hcp-bcc transformation [637].

Finally, as discussed in Section 9, an important task for the future is to create computational capabilities for direct MD simulations of the solute drag effect on interface motion. The initial step could involve a fast-diffusing inter- 
stitial impurity, such as $\mathrm{H}$ or $\mathrm{C}$, provided that a reasonably accurate potential for the relevant binary system will be developed.

\section{Acknowledgements}

We are grateful to Roy Benedek and Vasek Vitek for carefully reading the manuscript and making very useful suggestions. We are also grateful to Vesselin Yamakov for providing additional literature references and very helpful comments on simulations of grain boundary fracture. Y.M. acknowledges support of the US Department of Energy (Grant No. DE-FG02-01ER45871) and NASA (Grant No. NNX08AC07A). M.A. acknowledges support of the US Department of Energy (Grant No. DE-FG0206ER46282). Y.M. and M.A. also acknowledge support through the DOE Computational Materials Science Network Program. J.L. acknowledges support by ONR N00014-05-1-0504, NSF CMMI-0728069 and MRSEC grant DMR-0520020, and AFOSR FA9550-08-1-0325.

\section{References}

[1] Yip S, editor. Handbook of materials modeling. Dordrecht: Springer; 2005.

[2] Hafner J. Acta Mater 2000;48:71.

[3] Kobayashi R, Nakamura T, Ogata S. J Comput Theor Nanosci 2008;5:1768.

[4] Voter AF. J Chem Phys 1997;106:4665.

[5] Voter AF. Phys Rev B 1998;57:R13985.

[6] Sørensen MR, Voter AF. J Chem Phys 2000;112:9599.

[7] Laio A, Parrinello M. Proc Natl Acad Sci USA 2002;99:12562.

[8] Uberuaga BP, Stuart SJ, Voter AF. Phys Rev B 2007;75:014301

[9] Rudd RE, Mason DR, Sutton AP. Prog Mater Sci 2007;52:319.

[10] Paper on phase-field methods of this cluster, Acta Materialia.

[11] Paper on the finite-element and other continuum method of this cluster, Acta Materialia.

[12] Sutton AP, Balluffi RW. Interfaces in crystalline materials. Oxford: Clarendon Press; 1995.

[13] Hohenberg P, Kohn W. Phys Rev 1964;136:B864.

[14] Kohn W, Sham LJ. Phys Rev 1965;140:A1133.

[15] Qian XF, Li J, Lin X, Yip S. Phys Rev B 2006;73:035408.

[16] Horsfield AP, Bowler DR, Fisher AJ, Todorov TN, Sanchez CG. J Phys Condes Matter 2005; 17:4793.

[17] Car R, Parrinello M. Phys Rev Lett 1985;55:2471.

[18] Kresse G, Hafner J. Phys Rev B 1993;47:558.

[19] Laasonen K, Pasquarello A, Car R, Lee C, Vanderblit D. Phys Rev B 1993;47:10142.

[20] Mills G, Jonsson H, Schenter GK. Surf Sci 1995;324:305.

[21] Schmitt UW, Voth GA. J Chem Phys 1999;111:9361.

[22] Szabo A, Ostlund N. Modern quantum chemistry: introduction to advanced electronic structure theory. New York: McGraw-Hill; 1989.

[23] Ceperley DM, Alder BJ. Phys Rev Lett 1980;45:566.

[24] Vosko SH, Wilk L, Nusair M. Can J Phys 1980;58:1200.

[25] Perdew JP, Zunger A. Phys Rev B 1981;23:5048

[26] Perdew JP, Wang Y. Phys Rev B 1992;45:13244.

[27] Perdew JP, Chevary JA, Vosko SH, Jackson KA, Pederson MR, Singh DJ, et al. Phys Rev B 1992;46:6671.

[28] Perdew JP, Burke K, Ernzerhof M. Phys Rev Lett 1996;77:3865.

[29] Perdew JP, Burke K, Ernzerhof M. Phys Rev Lett 1997;78:1396.

[30] Stephens PJ, Devlin FJ, Chabalowski CF, Frisch MJ. J Phys Chem 1994;98:11623.

[31] Tao JM, Perdew JP, Staroverov VN, Scuseria GE. Phys Rev Lett 2003;91:146401.
[32] Kresse G, Furthmueller J. Vienna ab initio simulation package (VASP). Vienna: Vienna University; 2001.

[33] Kresse G, Furthmueller J. Phys Rev B 1996;54:11169.

[34] Gonze X, Beuken JM, Caracas R, Detraux F, Fuchs M, Rignanese GM, et al. Comput Mater Sci 2002;25:478.

[35] Baroni S, Corso AD, de Gironcoli S, Giannozzi P, Cavazzoni C, Ballabio G, et al. PWscf code website: http://www.pwscf.org/.

[36] Dacapo code homepage: http://wiki.fysik.dtu.dk/dacapo/Dacapo.

[37] Quantumespresso code homepage: http://www.quantum-espresso.org/.

[38] Schwarz K, Blaha P, Madsen GKH. Comput Phys Commun 2002;147:71.

[39] GAUSSIAN code homepage: http://www.lrz-muenchen.de/services/ software/chemie/gaussian/.

[40] GAMESS code homepage: http://www.msg.ameslab.gov/ GAMESS/

[41] NWChem code homepage: http://www.emsl.pnl.gov/docs/nwchem/ nwchem.html.

[42] Q-Chem code homepage: http://www.q-chem.com/.

[43] Soler JM, Artacho E, Gale JD, Garcia A, Junquera J, Ordejon P, et al. J Phys Condes Matter 2002;14:2745.

[44] Lewis JP, Glaesemann KR, Voth GA, Fritsch J, Demkov AA, Ortega J, et al. Phys Rev B 2001;64:195103.

[45] Chelikowsky JR, Troullier N, Saad Y. Phys Rev Lett 1994;72:1240.

[46] Pask JE, Sterne PA. Modell Simul Mater Sci Eng 2005;13:R71.

[47] Mortensen JJ, Hansen LB, Jacobsen KW. Phys Rev B 2005;71:035109.

[48] Troullier N, Martins JL. Phys Rev B 1991;43:1993.

[49] Vanderbilt D. Phys Rev B 1990;41:7892.

[50] Laasonen K, Car R, Lee C, Vanderbilt D. Phys Rev B 1991;43:6796.

[51] Blochl PE. Phys Rev B 1994;50:17953.

[52] Nielsen OH, Martin RM. Phys Rev B 1985;32:3780.

[53] Baroni S, de Gironcoli S, Dal Corso A, Giannozzi P. Rev Mod Phys 2001;73:515.

[54] Ogata S, Li J, Yip S. Science 2002;298:807.

[55] Ogata S, Li J, Hirosaki N, Shibutani Y, Yip S. Phys Rev B 2004;70:104104.

[56] Liu F, Ming PM, Li J. Phys Rev B 2007;76:064120.

[57] Sanchez JM, Ducastelle F, Gratias D. Physica A 1984;128:334.

[58] Zarkevich NA, Johnson DD. Phys Rev Lett 2004;92:255702.

[59] Fähnle M, Drautz R, Lechermann F, Singer R, Díaz-Ortiz A, Dosch H. Phys Stat Solidi B 2005;242:1159.

[60] Hart GLW, Blum V, Walorski MJ, Zunger A. Nat Mater 2005;4:391

[61] Sluiter MHF, Kawazoe Y. Phys Rev B 2005;71:212201.

[62] Díaz-Ortiz A, Dosch H, Drautz R. J Phys Condens Matter 2007;19:406206.

[63] van de Walle A, Ghosh G, Asta M. In: Bozzolo G, Noebe RD, Abel $P$, editors. Applied computational materials modeling. New York: Springer; 2007. p. 1-34.

[64] Ruban AV, Abrikosov IA. Rep Prog Phys 2008;71:046501.

[65] van de Walle A. Nat Mater 2008;7:455.

[66] van de Walle A. Calphad 2009;33:266.

[67] Stocks GM, Nicholson DM, Shelton WA, Gyorffy BL, Pinski FJ, Johnson DD. In: Gonis A, Turchi PEA, editors. Statics and dynamics of alloy phase transformations. NATO Advanced Study Institute, Series B: Physics. New York: Plenum Press; 1994.

[68] Vitos L, Abrikosov IA, Johansson B. Phys Rev Lett 2001;87:156401.

[69] Chepulskii RV, Staunton JB, Bruno E, Ginatempo B, Johnson DD. Phys Rev B 2002;65:064201.

[70] Rowlands DA, Ernst A, Gyorffy BL, Staunton JB. Phys Rev B 2006;73:165122.

[71] Geng HY, Sluiter MHF, Chen NX. J Chem Phys 2005;122:214706.

[72] van de Walle A, Ceder G. Rev Mod Phys 2002;74:11.

[73] Ozolins V, Sadigh B, Asta M. J Phys Condens Matter 2005;17:2197.

[74] Zunger A, Wei SH, Ferreira LG, Bernard JE. Phys Rev Lett 1990;65:353. 
[75] Jiang C, Wolverton C, Sofo J, Chen LQ, Liu ZK. Phys Rev B 2004;69:214202.

[76] Shin D, Arroyave R, Liu ZK, van de Walle A. Phys Rev B 2006; 74:024204.

[77] Shin D, van de Walle A, Wang Y, Liu ZK. Phys Rev B 2007;76:144204.

[78] Ghosh G, van de Walle A, Asta M. Acta Mater 2008;56:3202.

[79] Wolverton C, Zunger A. Phys Rev Lett 1995;75:3162.

[80] Shchyglo O, Bugaev VN, Drautz R, Udyansky A, Reichert H, Dosch HD. Phys Rev B 2005;72:140201.

[81] Geng HY, Sluiter MHF, Chen NX. Phys Rev B 2006;73:012202.

[82] Craievich PJ, Weinert M, Sanchez JM, Watson RE. Phys Rev Lett 1994;72:3076.

[83] Craievich PJ, Sanchez JM, Watson RE, Weinert M. Phys Rev B 1997;55:787.

[84] Grimvall G. Der Bunsen Phys Chem 1998;102:1083.

[85] Drautz R, Reichert H, Fähnle M, Dosch H, Sanchez JM. Phys Rev Lett 2001;87:236102.

[86] Müller S, Stöhr M, Wieckhorst O. Appl Phys A 2005;82:415.

[87] Han BC, Van der Ven A, Ceder G, Hwang BJ. Phys Rev B 2005;72:205409.

[88] Müller S. Surf Interface Anal 2006;38:1158.

[89] Liu JZ, van de Walle A, Ghosh G, Asta M. Phys Rev B 2005;72:144109.

[90] Van der Ven A, Ceder G. Phys Rev Lett 2005;94:045901.

[91] Van der Ven A, Thomas JC, Qingchuan X, Swoboda B, Morgan D. Phys Rev B 2008;78:104306.

[92] Kattner UR. JOM 1997;49:14

[93] Lukas HL, Fries SG, Sundman B. Computational thermodynamics. The Calphad method. Cambridge: Cambridge University Press; 2007.

[94] Ghosh G, van de Walle A, Asta M, Olson GB. Calphad 2002;26:491.

[95] Ghosh G, Olson GB. Acta Mater 2007;55:3281.

[96] Liu ZK, Chen LQ. In: Bozzolo G, Noebe RD, Abel P, editors. Applied computational materials modeling. New York: Springer; 2007.

[97] Turchi PEA, Abrikosov IA, Burton B, Fries SG, Grimvall G, Kaufman L, et al. Calphad 2007;31:4.

[98] Wolverton C, Yan ZY, Vijayaraghavan R, Ozolins V. Acta Mater 2002;50:2187.

[99] Mishin Y. In: Yip S, editor. Handbook of materials modeling. Dordrecht: Springer; 2005. p. 459-78.

[100] Finnis MW, Sinclair JE. Philos Mag A 1984;50:45.

[101] Daw MS, Baskes MI. Phys Rev B 1984;29:6443.

[102] Daw MS. In: Vitek V, Srolovitz DJ, editors. Atomistic simulation of materials: beyond pair potentials. New York: Plenum Press; 1989. p. $181-91$.

[103] Ackland GJ, Finnis MW, Vitek V. J Phys F: Met Phys 1988;18:L153.

[104] Mishin Y. Acta Mater 2004;52:1451.

[105] Williams PL, Mishin Y, Hamilton JC. Modell Simul Mater Sci Eng 2006; $14: 817$

[106] Voter AF, Chen SP. MRS Symp Proc 1987;82:175.

[107] Ercolessi F, Adams JB. Europhys Lett 1994;26:583.

[108] Mishin Y, Farkas D, Mehl MJ, Papaconstantopoulos DA. Phys Rev B 1999;59:3393.

[109] Foiles SM, Baskes MI, Daw MS. Phys Rev B 1986;33:7983.

[110] Mishin Y, Mehl MJ, Papaconstantopoulos DA, Voter AF, Kress JD. Phys Rev B 2001;63:224106.

[111] Mendelev MI, Han S, Srolovitz DJ, Ackland GJ, Sun DY, Asta M. Philos Mag 2003;83:3977.

[112] Mishin Y, Mehl MJ, Papaconstantopoulos DA. Phys Rev B 2002;65:224114.

[113] Farkas D, Rocqueta D, Vilette A, Ternes K. Modell Simul Mater Sci Eng 1996;4:359.

[114] Landa A, Wynblatt P, Gerschick A, Vitek V, Ruban A, Skriver H. Acta Mater 1999;47:2477.
[115] Tomar V, Zhou M. Phys Rev B 2006;73:174116.

[116] Zope RR, Mishin Y. Phys Rev B 2003;68:024102.

[117] Strachan A, Cagin T, Gülseren O, Mukherjee S, Cohen RE, Goddard WA. Modell Simul Mater Sci Eng 2004;12:S445.

[118] Mishin Y, Mehl MJ, Papaconstantopoulos DA. Acta Mater 2005;53:4029.

[119] Mishin Y, Lozovoi AY. Acta Mater 2006;54:5013.

[120] Chamati H, Papanicolaou N, Mishin Y, Papaconstantopoulos DA. Surf Sci 2006;600:1793.

[121] Mendelev MI, Kramer MJ, Becker CA, Asta M. Philos Mag 2008;68:1723.

[122] Robertson IJ, Heine V, Payne MC. Phys Rev Lett 1993;70:1944.

[123] Frederiksen SL, Jacobsen KW, Brown KS, Sethna JP. Phys Rev Lett 2004;93:165501.

[124] Behler J, Parrinello M. Phys Rev Lett 2007;98:146401.

[125] Sanville E, Bholoa A, Smith R, Kenny SD. J Phys Condens Matter 2008;20:285219.

[126] Hunger J, Beyreuther S, Huttner G, Allinger K, Radelof U, Zsoinal L. Eur J Inorg Chem 1998;6:693.

[127] Marques JMC, Prudente FV, Pereira FB, Almeida MM, Maniero AM, Fellows CE. J Phys B At Mol Opt Phys 2008;41:085103.

[128] Brommer P, Gahler F. Modell Simul Mater Sci Eng 2007;15:295.

[129] Brenner DW. Phys Stat Solidi B 2000;217:23.

[130] Baskes MI. Phys Rev Lett 1987;59:2666.

[131] Baskes MI, Nelson JS, Wright AF. Phys Rev B 1989;40:6085.

[132] Baskes MI. Phys Rev B 1992;46:2727.

[133] Baskes MI, Angelo JE, Bisson CL. Modell Simul Mater Sci Eng 1994;2:505.

[134] Baskes MI. Mater Chem Phys 1997;50:152.

[135] Lee BJ, Shim JH, Baskes MI. Phys Rev B 2003;68:144112.

[136] Baskes MI, Srinivasan SG, Valone SM, Hoagland RG. Phys Rev B 2007;75:094113

[137] Baskes MI, Johnson RA. Modell Simul Mater Sci Eng 1994;2:147.

[138] Kim YM, Lee BJ, Baskes MI. Phys Rev B 2006;74:014101.

[139] Lee BJ, Baskes MI, Kim H, Cho YK. Phys Rev B 2001;64:184102.

[140] Baskes MI. Mater Sci Eng A 1999;261:165.

[141] Chen D, Yan M, Liu YF. Scripta Mater 1999;40:913.

[142] Hu SY, Baskes MI, Stan M, Chen LQ. Acta Mater 2006;54:4699.

[143] Jelinek B, Houze J, Kim S, Horstemeyer MF, Baskes MI, Kim SG. Phys Rev B 2007;75:054106.

[144] Sa I, Lee BJ. Scripta Mater 2008;59:595.

[145] Lee BJ, Lee WL. Calphad 2005;29:7.

[146] Lee BJ. Acta Mater 2006;54:701.

[147] Lee BJ, Jang JW. Acta Mater 2007;55:6779.

[148] Kim YM, Lee BJ. Acta Mater 2008;56:3481.

[149] Hashibon A, Lozovoi AY, Mishin Y, Elsässer C, Gumbsch P. Phys Rev B 2008;77:094131.

[150] Pasianot R, Farkas D, Savino EJ. Phys Rev B 1991;43:6952.

[151] Oliver S, Conte R, Fortunelli A. Phys Rev B 2008;77:054104.

[152] Webb EB, Grest GS. Phys Rev Lett 2001;86:2066.

[153] Wu G, Lu G, Garcia-Cervera CJ, E W. Phys Rev B 2009;79: 035124.

[154] Krasko GL. J Appl Phys 1996;79:4682.

[155] Dudarev SL, Derlet PM. J Phys Condens Matter 2005;17:7097.

[156] Dudarev SL, Derlet PM. J Comput Aided Mater Des 2007;14: 129.

[157] Finnis MW. Interatomic forces in condensed matter. Oxford: Oxford University Press; 2003.

[158] Stillinger FH, Weber TA. Phys Rev B 1985;31:5262.

[159] Tersoff J. Phys Rev B 1988;37:6991.

[160] Bazant MZ, Kaxiras E. Phys Rev B 1997;56:8542.

[161] Broughton JQ, Mehl MJ. Phys Rev B 1999;59:9259.

[162] Muller M, Ehrhart P, Albe K. J Phys Condens Matter 2007;19:326220.

[163] Haile JM. Molecular dynamics simulation: elementary methods. New York: Wiley; 1997.

[164] Rapaport DC. The art of molecular dynamics simulation. 2nd ed. Cambridge: Cambridge University Press; 2004. 
[165] Li J. In: Yip S, editor. Handbook of materials modeling. Dordrecht: Springer; 2005. p. 565-88 [Mistake free version at http:// alum.mit.edu/www/liju99/Papers/05/Li05-2.8.pdf].

[166] Allen M, Tildesley D. Computer simulation of liquids. New York: Clarendon Press; 1987.

[167] Schlick T. Molecular modeling and simulation. Berlin: SpringerVerlag; 2002

[168] Frenkel D, Smit B. Understanding molecular simulation: from algorithms to applications. 2nd ed. San Diego, CA: Academic Press; 2002.

[169] Li J, Ngan AHW, Gumbsch P. Acta Mater 2003;51:5711.

[170] Li J. In: Yip S, editor. Handbook of materials modeling. Dordrecht: Springer; 2005. p. 773-92 [Mistake free version at http:// alum.mit.edu/www/liju99/Papers/05/Li05-2.19.pdf].

[171] Stillinger FH. Science 1995;267:1935.

[172] Mauro JC, Loucks RJ, Balakrishnan J, Raghavan S. J Chem Phys 2007;126:194103.

[173] Massen CP, Doye JPK. Phys Rev E 2007;75:037101.

[174] Rickman JM, LeSar R. Annu Rev Mater Res 2002;32:195.

[175] van de Walle A, Ceder G. Rev Mod Phys 2002;74:11.

[176] Dmitriev SV, Kitamura T, Li J, Umeno Y, Yashiro K, Yoshikawa N. Acta Mater 2005;53:1215.

[177] Li J, Yip S. Phys Rev B 1997;56:3524.

[178] Porter LJ, Li J, Yip S. J Nucl Mater 1997;246:53.

[179] Li J, Porter L, Yip S. J Nucl Mater 1998;255:139.

[180] Torrie GM, Valleau JP. J Comput Phys 1977;23:187.

[181] Newman MEJ, Barkema GT. Monte Carlo methods in statistical physics. Oxford: Clarendon Press; 1999.

[182] Landau DP, Binder K. A guide to Monte Carlo simulations in statistical physics. Cambridge: Cambridge University Press; 2000.

[183] Binder K, Heermann DW. Monte Carlo simulation in statistical physics. 4th ed. Berlin: Springer-Verlag; 2002.

[184] Wang FG, Landau DP. Phys Rev Lett 2001;86:2050.

[185] Fichthorn KA, Weinberg WH. J Chem Phys 1991;95:1090.

[186] Bales GS, Chrzan DC. Phys Rev B 1994;50:6057.

[187] Soneda N, de la Rubia TD. Philos Mag A: Phys Condens Matter Struct Defect Mech Prop 1998;78:995.

[188] Sadigh B, Lenosky TJ, Theiss SK, Caturla MJ, de la Rubia TD, Foad MA. Phys Rev Lett 1999;83:4341.

[189] Vineyard GH. Phys Chem Solid 1957;3:121.

[190] Bulatov VV, Hsiung LL, Tang M, Arsenlis A, Bartelt MC, Cai W, et al. Nature 2006;440:1174.

[191] Bulatov VV, Argon AS. Modell Simul Mater Sci Eng 1994;2:167.

[192] Voter AF, Montalenti F, Germann TC. Annu Rev Mater Res 2002;32:321.

[193] Li J. MRS Bull 2007;32:151.

[194] Henkelman G, Uberuaga BP, Jonsson H. J Chem Phys 2000;113:9901.

[195] Henkelman G, Jonsson H. J Chem Phys 2000;113:9978.

[196] Zhu T, Li J, Yip S. Phys Rev Lett 2004;93:025503.

[197] Zhu T, Li J, Yip S. Phys Rev Lett 2004;93:205504.

[198] Zhu T, Li J, Lin X, Yip S. J Mech Phys Solids 2005;53:1597.

[199] Henkelman G, Jonsson H. J Chem Phys 1999;111:7010.

[200] Zhu T, Li J, Samanta A, Kim HG, Suresh S. Proc Natl Acad Sci USA 2007;104:3031.

[201] Zhu T, Li J, Samanta A, Leach A, Gall K. Phys Rev Lett 2008;100:025502.

[202] Barkema GT, Mousseau N. Phys Rev Lett 1996;77:4358.

[203] Miron RA, Fichthorn KA. J Chem Phys 2003;119:6210.

[204] Miron RA, Fichthorn KA. Phys Rev Lett 2004;93:128301.

[205] Laio A, Parrinello M. Proc Natl Acad Sci USA 2002;99:12562.

[206] Laio A, Rodriguez-Fortea A, Gervasio FL, Ceccarelli M, Parrinello M. J Phys Chem B 2005;109:6714.

[207] Abraham FF, Walkup R, Gao HJ, Duchaineau M, De la Rubia TD, Seager M. Proc Natl Acad Sci USA 2002;99:5783.

[208] Buehler MJ, Abraham FF, Gao HJ. Nature 2003;426:141.
[209] Kadau K, Germann TC, Hadjiconstantinou NG, Lomdahl PS, Dimonte G, Holian BL, et al. Proc Natl Acad Sci USA 2004;101:5851.

[210] Nakano A, Kalia RK, Nomura K, Sharma A, Vashishta P, Shimojo F, et al. Comput Mater Sci 2007;38:642.

[211] Germann TC, Kadau K. Int J Mod Phys C 2008;19:1315.

[212] Plimpton S. J Comput Phys 1995;117:1.

[213] Stadler J, Mikulla R, Trebin HR. Int J Mod Phys C 1997;8:1131.

[214] Smith W, Forester TR. J Mol Graph 1996;14:136.

[215] Wolf D, Yip S, editors. Materials interfaces atomic-level structure and properties. London: Chapman \& Hall; 1992.

[216] Sutton AP, Vitek V. Philos Trans Roy Soc Lond A 1983;309:37.

[217] Rohrer GS, Saylor DM, El Dasher B, Adams B, Rollett AD, Wynblatt P. Z Metallk 2004;95:197.

[218] Saylor DM, El Dasher BS, Rollett AD, Rohrer GS. Acta Mater 2004;52:3649.

[219] Kim CS, Rollett AD, Rohrer GS. Scripta Mater 2006;54:1005.

[220] Medlin DL, Foiles SM, Cohen D. Acta Mater 2001;49:3689.

[221] Goukon N, Yamada T, Kajihara M. Acta Mater 2000;48:2837.

[222] Lee SB. Mater Lett 2003;57:3779.

[223] Duparc OH, Poulat S, Larere A, Thibault J, Priester L. Philos Mag A 2000;80:853.

[224] Merkle KL. Interface Sci 1995;2:311.

[225] Merkle KL, Thompson LJ, Phillips F. Interface Sci 2004;12:277.

[226] Hamilton JC, Siegel DJ, Daruka I, Léonard F. Phys Rev Lett 2003;90:246102.

[227] Schmidt C, Ernst F, Finnis MW, Vitek V. Phys Rev Lett 1995;75:2160.

[228] Schmidt C, Finnis MW, Ernst F, Vitek V. Philos Mag A 1998;77:1161.

[229] Sansoz F, Molinari JF. Acta Mater 2005;53:1931.

[230] Spearot DE, Tschopp MA, Jacob KI, McDowell DL. Acta Mater 2007;55:705.

[231] Tschopp MA, Tucker GJ, McDowell DL. Acta Mater 2007;55:3959.

[232] Tschopp MA, McDowell DL. Philos Mag 2007;87:3147.

[233] Tschopp MA, McDowell DL. Philos Mag 2007;87:3871.

[234] Brown JA, Mishin Y. Phys Rev B 2007;76:134118.

[235] Wu ZX, Zhang YW, Srolovitz DJ. Acta Mater 2009;57:4278.

[236] Campbell GH, Chan DK, Medlin DL, Anelo JE, Carter CB. Scripta Mater 1996;35:837.

[237] Nadarzinski K, Ernst F. Philos Mag A 1996;74:641.

[238] Medlin DL, Campbell GH, Carter CB. Acta Mater 1998;46:5135.

[239] Merkle KL. Ultramicroscopy 1991;37:130.

[240] Merkle KL, Wolf D. Philos Mag A 1992;65:513.

[241] Cahn JW. J Phys Colloques 1982;43:199.

[242] Hsieh TE, Balluffi RW. Acta Metall 1989;37:2133.

[243] Marquis EA, Hamilton JC, Medlin DL, Leonard F. Phys Rev Lett 2004;93:156101.

[244] Hashimoto M, Ishida Y, Yamamoto R, Doyama M. Acta Metall 1981;29:617.

[245] Broughton JQ, Gilmer GH. J Chem Phys 1986;84:5759.

[246] Broughton JQ, Gilmer GH. J Phys Chem 1987;91:6347.

[247] Najafabadi R, Srolovitz DJ, LeSar R. J Mater Res 1991;6:999.

[248] Rickman JM, Najafabadi R, Zhao L, Srolovitz DJ. J Phys Condens Matter 1992;4:4923.

[249] Sutton AP, Mulheran PA, Stoneham AM. Philos Trans R Soc Lond A 1992;341:233.

[250] Hairie A, Lebouvier B, Nouet G, Paumier E, Ralantoson N, Sutton AP. Interface Sci 1994;2:17.

[251] Foiles SM. Phys Rev B 1994;49:14930.

[252] Broughton JQ, Gilmer GH. Modell Simul Mater Sci Eng 1998;6:393.

[253] Hou M, Hairie M, Lebouvier B, Paumier E, Ralantoson N, Duparc OH, et al. Mater Sci Forum 1996;207-209:249.

[254] Lusk MT, Fellinger MR, Beale PD. J Chem Phys 2006;124:064707.

[255] Herring C. In: Gomer R, Smith CS, editors. The physics of powder metallurgy. New York: McGraw-Hill; 1949. 
[256] Herring C. In: Gomer R, Smith CS, editors. Structure and properties of solid surfaces. Chicago, IL: University of Chicago Press; 1953. p. 5-81.

[257] Du D, Zhang H, Srolovitz DJ. Acta Mater 2007;55:467.

[258] Traut ZT, Upmanyu M. Scripta Mater 2005;52:1175.

[259] Foiles SM, Hoyt JJ. Acta Mater 2006;54:3351.

[260] Rice JR, Wang JS. Mater Sci Eng A 1989;107:23

[261] Wu R, Freeman AJ, Olson GB. Science 1994:265:376.

[262] Zhong L, Wu R, Freeman AJ, Olson GB. Phys Rev B 2000;62:13938

[263] Braithwaite JS, Rez P. Acta Mater 2005;53:2715.

[264] Wachowicz E, Kiejna A. Comput Mater Sci 2008;43:736.

[265] Yamaguchi M, Shiga M, Kaburaki H. J Phys Condens Matter 2004;16:3933

[266] Yamaguchi M, Nishiyama Y, Kaburaki H. Phys Rev B 2007;76:035418

[267] Geng WT, Freeman AJ, Olson GB. Phys Rev B 2001;63:165415.

[268] Geng WT, Freeman AJ, Wu R, Geller CB, Raynolds JE. Phys Rev B 1999;60:7149.

[269] Smith RW, Geng WT, Geller CB, Wu R, Freeman AJ. Scripta Mater 2000;43:957.

[270] Janisch R, Elsässer C. Phys Rev B 2003;67:224101.

[271] Kim M, Geller CB, Freeman AJ. Scripta Mater 2004;50:1341

[272] Gesari S, Irigoyen B, Juan A. Appl Surf Sci 2006;253:1939.

[273] Duscher G, Chisholm MF, Alber U, Ruhle M. Nat Mater 2004;3:621.

[274] Schweinfest R, Paxton AT, Finnis MW. Nat Mater 2004;432:1008.

[275] Lozovoi AY, Paxton AT, Finnis MW. Phys Rev B 2006;74:155416.

[276] Lozovoi AY, Paxton AT. Phys Rev B 2008;77:165413.

[277] Thomson DI, Heine V, Finnis MW, Marzari N. Philos Mag Lett 1997;76:281

[278] Thomson DI, Heine V, Payne MC, Marzari N, Finnis MW. Acta Mater 2000;48:3623.

[279] Zhang Y, Lu GH, Wang T, Deng S, Shu X, Kohyama M, et al. J Phys Condens Matter 2006;18:5121.

[280] Stumpf R, Feibelman PJ. Phys Rev B 1996;54:5145.

[281] Buban JP, Matsunada K, Chen J, Shibata N, Ching WY, Yamamoto T, et al. Science 2006;311:212.

[282] Foiles SM. In: Surface segregation phenomena. Boca Raton, FL: CRC Press; 1990. p. 79.

[283] Udler D, Seidman DN. Phys Stat Solidi B 1992;172:267.

[284] Udler D, Seidman DN. Acta Mater 1994;42:1959.

[285] Menyhard M, Yan M, Vitek V. Acta Metall Mater 1994:42:2783.

[286] Rittner JD, Seidman DN. Acta Mater 1997;45:3191.

[287] Liu XY, Xu W, Foiles SM, Adams JB. Appl Phys Lett 1998;72:1578.

[288] Creuze J, Berthier F, Tetot R, Legrand B. Phys Rev B 2000;62:2813.

[289] Creuze J, Berthier F, Tetot R, Legrand B. Phys Rev Lett 2001;86:5735.

[290] Xie X, Mishin Y. Acta Mater 2002;50:4303.

[291] Brown JA, Mishin Y. Acta Mater 2005;53:2149.

[292] Williams PL, Mishin Y. Acta Mater 2009;57:3786.

[293] Millett PC, Selvan RP, Saxena A. Acta Mater 2007;55:2329.

[294] Wang Y, Liu ZK, Chen LQ, Wolverton C. Acta Mater 2007;55:5934.

[295] Hashibon A, Elsässer C, Mishin Y, Gumbsch P. Phys Rev B 2007;76:245434.

[296] Vaithyanathan V, Wolverton C, Chen LQ. Phys Rev Lett 2002;88:125503.

[297] Vaithyanathan V, Wolverton C, Chen LQ. Acta Mater 2004;52:2973

[298] Benedek R, Seidman DN, Woodward C. J Phys Condens Matter 2002;14:2877.

[299] Benedek R, Seidman DN, Woodward C. Interface Sci 2004;12:57.

[300] Marquis EA, Seidman DN, Asta M, Woodward C, Ozolins V. Phys Rev Lett 2003;91:036101.

[301] Marquis EA, Seidman DN, Asta M, Woodward C. Acta Mater 2006:54:119.

[302] Peng P, Soh AK, Yang R, Hu ZQ. Comput Mater Sci 2006;38:354.
[303] Zhou Y, Mao Z, Booth-Morrison C, Seidman DN. Appl Phys Lett 2008;93:171905.

[304] Amouyal Y, Mao Z, Seidman DN. Appl Phys Lett 2008;93:201905.

[305] Wang C, Wang CY. Surf Sci 2008;602:2604.

[306] Wang YJ, Wang CY. J Appl Phys 2008;104:013109.

[307] Wang YJ, Wang CY. Mater Sci Eng A 2008;490:242.

[308] Geng WT, Ping DH, Gu YF, Cui CY, Harada H. Phys Rev B 2007;76:224102.

[309] Liu Y, Chen KY, Lu G, Zhang JH, Hu ZQ. Acta Mater 1997;45:1837.

[310] Geng CY, Wang CY, Yu T. Physica B 2005;358:314.

[311] Peng P, Zhou DW, Liu JS, Yang R, Hu ZQ. Mater Sci Eng A 2006;416:169.

[312] Wu YX, Li XY, Wang YM. Acta Mater 2007;55:4845.

[313] Sluiter M, Kawazoe Y. Phys Rev B 1996;54:10381.

[314] Asta M, Foiles SM, Quong AA. Phys Rev B 1998:57:11265.

[315] Asta M, Hoyt JJ. Acta Mater 2000;48:1089.

[316] Asta M, Ozolins V, Woodward C. JOM 2001;9:16.

[317] Ardell AJ, Ozolins V. Nat Mater 2005;4:309.

[318] van de Walle A, Ellis DE. Phys Rev Lett 2007;98:266101.

[319] Hyland RW, Asta M, Rohrer CL, Foiles SM. Acta Mater 1998:46:3667.

[320] Becker CA, Mishin Y, Boettinger WJ. J Mater Sci 2008;43:3873.

[321] Asta M. Acta Mater 1996;44:4131.

[322] Kurz W, Fisher DJ. Fundamentals of solidification. Rockport, MA: Trans Tech Publications; 1984.

[323] Tiller WA. The science of crystallization: microscopic interfacial phenomena. New York: Cambridge University Press; 1991.

[324] Herlach D, Galenko P, Holland-Moritz D. Metastable solids from undercooled melts. Boston, MA: Elsevier; 2007.

[325] Hoyt JJ, Asta M, Karma A. Mater Sci Eng R 2003;41:121.

[326] Hoyt JJ, Asta M, Haxhimali T, Karma A, Napolitano R, Trivedi R, et al. MRS Bull 2004;29:935.

[327] Laird BB, Davidchack RL. J Phys Chem B 2005;109:17802.

[328] Asta M, Beckermann C, Karma A, Kurz W, Napolitano R, Plapp M, et al. Acta Mater 2009;57:941.

[329] Davidchack RL, Laird BB. Phys Rev E 1996;54:5905.

[330] Davidchack RL, Laird BB. Mol Phys 1999;97:833.

[331] Sibug-Aga R, Laird BB. J Chem Phys 2002;116:3410.

[332] Sibug-Aga R, Laird BB. Phys Rev B 2002;66:144106.

[333] Becker CA, Asta M, Hoyt JJ, Foiles SM. J Chem Phys 2006; 124:164708.

[334] Becker CA, Olmsted DL, Asta M, Hoyt JJ, Foiles SM. Phys Rev B 2009;79:054109.

[335] Frolov T, Mishin Y. Phys Rev B 2009;79:045430.

[336] Ramalingam H, Asta M, van de Walle A, Hoyt JJ. Interface Sci 2002;10:149

[337] Frolov T, Mishin Y. J Chem Phys 2009;131:054702.

[338] Asta M, Hoyt JJ, Karma A. Phys Rev B 2002;66:R100101.

[339] Becker CA, Olmsted DL, Asta M, Hoyt JJ, Foiles SM. Phys Rev Lett 2007:98:125701.

[340] Amini M, Laird BB. Phys Rev B 2008;78:144112.

[341] Hoyt JJ, Asta M, Karma A. Phys Rev Lett 2001;86:5530.

[342] Haxhimali T, Karma A, Gonzales F, Rappaz M. Nat Mater 2006;5:660.

[343] Geysermans P, Gorse D, Pontikis V. J Chem Phys 2000;113:6382.

[344] Geysermans P, Pontikis V. J Phys IV France 2002;12:238.

[345] Heni M, Löwen H. Phys Rev E 2002;65:021501.

[346] Kaplan WD, Kauffmann Y. Annu Rev Mater Res 2006;36:1.

[347] Jin ZH, Gumbsch P, Ma E, Albe K, Lu K, Hahn H, et al. Scripta Mater 2006;54:1163

[348] Jin ZH, Gumbsch P, Albe K, Ma E, Lu K, Gleiter H, et al. Acta Mater 2008;56:1126.

[349] Dewald MP, Curtin WA. Philos Mag 2007;87:4615.

[350] Cheng Y, Mrovec M, Gumbsch P. Philos Mag 2008;88:547.

[351] Cheng Y, Mrovec M, Gumbsch P. Mater Sci Eng A 2008:483:329.

[352] Rao SI, Hazzledine PM. Philos Mag A 2000;80:2011. 
[353] Hoagland RG, Mitchell TE, Hirth JP, Kung H. Philos Mag A 2002;82:643.

[354] Chen Z, Jin Z, Gao H. Phys Rev B 2007;75:212104.

[355] Hoagland RG, Kurtz RJ, Henager CH. Scripta Mater 2004;50:775.

[356] Wang J, Hoagland RG, Hirth JP, Misra A. Acta Mater 2008;56:3109.

[357] Wang J, Hoagland RG, Hirth JP, Misra A. Acta Mater 2008;56:5685.

[358] Kurtz RJ, Hoagland RG, Hirth JP. Philos Mag A 1999;79:665.

[359] Kurtz RJ, Hoagland RG, Hirth JP. Philos Mag A 1999;79:683.

[360] Hoagland RG, Kurtz RJ. Philos Mag A 2002;82:1073.

[361] Hirth JP. J Phys Chem Solids 1994;55:985.

[362] Hirth JP, Pond RC. Acta Mater 1996;44:4749.

[363] Sansoz F, Molinari JF. Acta Mater 2004;53:1283.

[364] Spearot DE, Jacob KI, McDowell DL. Acta Mater 2005;53:3579.

[365] Tschopp MA, McDowell DL. Scripta Mater 2008;58:299.

[366] Suzuki A, Mishin Y. Mater Sci Forum 2005;502:157.

[367] Cahn JW, Mishin Y, Suzuki A. Philos Mag 2006;86:3965.

[368] Cahn JW, Mishin Y, Suzuki A. Acta Mater 2006;54:4953.

[369] Mishin Y, Suzuki A, Uberuaga B, Voter AF. Phys Rev B 2007;75:224101.

[370] Ivanov VA, Mishin Y. Phys Rev B 2008;78:064106.

[371] Molteni C, Francis GP, Payne MC, Heine V. Phys Rev Lett 1996;76:1284

[372] Molteni C, Morzani N, Payne MC, Heine V. Phys Rev Lett 1997;79:869.

[373] Hamilton JC, Foiles SM. Phys Rev B 2002;65:064104.

[374] Chen LQ, Kalonji G. Philos Mag A 1992;66:11.

[375] Shiga M, Shinoda W. Phys Rev B 2004;70:054102.

[376] Chandra N, Dang P. J Mater Sci 1999;34:655.

[377] Haslam AJ, Moldovan D, Yamakov V, Wolf D, Phillpot SR, Gleiter H. Acta Mater 2003;51:2097.

[378] Li CH, Edwards EH, Washburn J, Parker J. Acta Metall 1953;1:223.

[379] Bainbridge DW, Li CH, Edwards EH. Acta Metall 1954;2:322.

[380] Biscondi M, Goux C. Mem Sci Rev Met 1968;75:167.

[381] Fukutomi H, Iseki T, Endo T, Kamijo T. Acta Mater 1991;39:1445.

[382] Winning M, Gottstein G, Shvindlerman LS. Acta Mater 2001;49:211.

[383] Winning M, Gottstein G, Shvindlerman LS. Acta Mater 2002;50:353.

[384] Winning M, Rollett AD. Acta Mater 2005;53:2901.

[385] Sheikh-Ali AD, Szpunar JA, Garmestani H. Interface Sci 2003;11:439.

[386] Yoshida H, Yokoyama K, Shibata N, Ikuhara Y, Sakuma T. Acta Mater 2004;52:2349.

[387] Legros M, Gianola DS, Hemker KJ. Acta Mater 2008;56:3380.

[388] Mompiou F, Caillard D, Legros M. Acta Mater 2009;57:2198.

[389] Molodov DA, Shvindlerman LS. Int J Mater Res 2009;100:461.

[390] Cahn JW, Taylor JE. Acta Mater 2004;52:4887.

[391] Cahn JW, Mishin Y. Int J Mater Res 2009;100:510.

[392] Taylor JE, Cahn JW. Interfaces Free Boundaries 2007;9:493.

[393] Srinivasan SG, Cahn JW. In: Ankem S, Pande CS, Ovidko I, Ranganathan R, editors. Science and technology of interfaces, Seattle 2002. Warrendale, PA: TMS; 2002. p. 3-14.

[394] Zhang H, Duy D, Srolovitz DJ. Philos Mag 2008;88:243.

[395] Molodov D, Ivanov A, Gottstein G. Acta Mater 2007;55:1843.

[396] Molodov D, Gorkaya T, Gottstein G. Mater Sci Forum 2007;558559:927.

[397] Winning M. Philos Mag 2007;87:5017.

[398] Monk J, Hyde B, Farkas D. J Mater Sci 2006;41:7741.

[399] Hemker KJ, Sharpe WN. Annu Rev Mater Res 2007;37:93.

[400] Gianola DS, Eberl C, Cheng XM, Hemker KJ. Adv Mater 2008;20:303.

[401] Bernstein N. Acta Mater 2008;56:1106.

[402] Mate CM, McClelland GM, Erlandsson R, Chiang S. Phys Rev Lett 1987;59:1942.

[403] Gnecco E, Bennewitz R, Gyalog T, Loppacher C, Bammerlin M, Meyer E, et al. Phys Rev Lett 2000;84:1172.
[404] Gnecco E, Bennewitz R, Gyalog T, Meyer E. J Phys Condens Matter 2001;13:R619.

[405] Socoliuc A, Gnecco E, Maier S, Pfeiffer O, Baratoff A, Bennewitz R, et al. Science 2006;313:207.

[406] Ivanov VA, Mishin Y. Phys Rev B 2008;78:064106.

[407] Uberuaga BP, Stuart SJ, Voter AF. Phys Rev B 2007;75:014301.

[408] Anderson PM, Rice JR. Scripta Metall 1986;20:1467.

[409] McMahon CJ, Vitek V. Acta Met 1979;27:507.

[410] Farkas D, Selinger R. In: Yip S, editor. Handbook of materials modeling. Dordrecht: Springer; 2005.

[411] Yip S, Wolf D. Mater Sci Forum 1989;46:77.

[412] Rudd RE, Belak JF. Comput Mater Sci 2002;24:148.

[413] Farkas D. Philos Mag A 2000;80:1425.

[414] Farkas D, Van Swygenhoven H, Derlet P. Phys Rev B 2002;66:060101.

[415] Cao A, Wei Y. Phys Rev B 2007;76:024113.

[416] Cleri F, Phillpot SR, Wolf D. Interface Sci 1999;7:45.

[417] Farkas D. Philos Mag Lett 2000;80:229.

[418] Yamakov V, Saether E, Phillips DR, Glaessgen EH. Phys Rev Lett 2005;95:015502.

[419] Yamakov V, Saether E, Glaessgen EH. J Mate Sci 2008;43:7488.

[420] Yamakov V, Saether E, Phillips DR, Glaessgen EH. J Mech Phys Solids 2006;54:1899.

[421] Coffman VR, Sethna JP, Heber G, Liu M, Ingraffea A, Bailey NP, et al. Modell Simul Mater Sci Eng 2008;16:065008.

[422] Zhou XW, Zimmermann JA, Reedy ED, Moody NR. Mech Mater 2008; $40: 832$.

[423] Mulford RA, McMahon CJ, Pope DP, Feng HC. Met Trans A Phys Met Mater Sci 1976;7:1183.

[424] Pugh SF. An introduction to grain boundary fracture in metals. London: The Institute of Metals; 1991.

[425] Liu CT, White CL, Horton JA. Acta Met 1985;33:213.

[426] Liu CT, Lee EH, McKamey CG. Scripta Metall 1989;23:875.

[427] Yamaguchi M, Umakoshi Y. Prog Mater Sci 1990;34:1.

[428] Liu CT. Scripta Metall Mater 1992;27:25.

[429] Liu CT, George EP, Maziasz PJ, Schneibel JH. Mater Sci Eng A: Struct Mater Prop Microstruct Process 1998;258:84.

[430] Latanision RM, Opperhauser H. Met Trans 1974;5:483.

[431] McMahon CJ. Eng Fract Mech 2001;68:773.

[432] Robertson IM, Tabata T, Wei W, Heubaum F, Birnbaum HK. Scripta Metall 1984;18:841.

[433] Muller DA, Mills MJ. Mater Sci Eng A: Struct Mater Prop Microstruct Process 1999;260:12.

[434] Misra RDK. Surf Interface Anal 2001;31:509.

[435] Teter DF, Robertson IM, Birnbaum HK. Acta Mater 2001;49:4313.

[436] Duscher G, Chisholm MF, Alber U, Ruhle M. Nat Mater 2004;3:621.

[437] Finnis MW. J Phys Condes Matter 1996;8:5811.

[438] Ogata S, Umeno Y, Kohyama M. Modell Simul Mater Sci Eng 2009;17:013001.

[439] Gumbsch P. Mater Sci Eng A 1999;260:72.

[440] Seah MP. Acta Met 1980;28:955.

[441] Hirth JP, Rice JR. Met Trans A Phys Met Mater Sci 1980;11:1501.

[442] Rice JR, Wang JS. Mater Sci Eng A: Struct Mater Prop Microstruct Process 1989;107:23.

[443] Bika D, McMahon CJ. Acta Metall Mater 1995;43:1909.

[444] Pfaendtner JA, McMahon CJ. Acta Mater 2001;49:3369.

[445] Lozovoi AY, Paxton AT, Finnis MW. Phys Rev B 2006;74:155416.

[446] Messmer RP, Briant CL. Acta Met 1982;30:457.

[447] Wu RQ, Freeman AJ, Olson GB. Science 1994;265:376.

[448] Losch W. Acta Met 1979;27:1885.

[449] Hashimoto M, Ishida Y, Wakayama S, Yamamoto R, Doyama M, Fujiwara T. Acta Met 1984;32:13.

[450] Eberhart ME, Vvedensky DD. Phys Rev Lett 1987;58:61.

[451] Krasko GL, Olson GB. Solid State Commun 1990;76:247.

[452] Krasko GL. Mater Sci Eng A: Struct Mater Prop Microstruct Process 1997;234:1071.

[453] Eberhart M. Science 1994;265:332. 
[454] Painter GS, Averill FW. Phys Rev Lett 1987;58:234.

[455] Kitamura T, Umeno Y. Modell Simul Mater Sci Eng 2003;11:127.

[456] Lu GH, Deng SH, Wang TM, Kohyama M, Yamamoto R. Phys Rev B 2004;69:134106.

[457] Yamaguchi M, Shiga M, Kaburaki H. Science 2005;307:393.

[458] Goodwin L, Needs RJ, Heine V. J Phys Condes Matter 1990;2:351.

[459] Lu G, Kioussis N, Wu R, Ciftan M. Phys Rev B 1999;59:891.

[460] Farkas D, Nogueira R, Ruda M, Hyde B. Metall Mater Trans A Phys Metall Mater Sci 2005;36A:2067.

[461] Lu GH, Zhang Y, Deng S, Wang T, Kohyama M, Yamamoto R, et al. Phys Rev B 2006;73:224115.

[462] Zhang Y, Lu GH, Deng SH, Wang TM, Xu HB, Kohyama M. Phys Rev B 2007;75:174101.

[463] Xiao W, Liu CS, Tian ZX, Geng WT. J Appl Phys 2008;104:053519.

[464] Kohyama M. Philos Mag Lett 1999;79:659.

[465] Kohyama M. Phys Rev B 2002;65:184107.

[466] Christensen M, Wahnstrom G. Acta Mater 2004;52:2199.

[467] Chen J, Xu YN, Rulis P, Ouyang LZ, Ching WY. Acta Mater 2005;53:403.

[468] Ogata S, Kitagawa H. J Jpn Inst Met 1996;60:1079.

[469] Qi Y, Hector LG. Phys Rev B 2003;68:201403.

[470] Qi Y, Hector LG. Phys Rev B 2004;69:235401.

[471] Zhang Q, Cagin T, van Duin A, Goddard WA, Qi Y, Hector LG. Phys Rev B 2004;69:045423.

[472] Dmitriev SV, Yoshikawa N, Kohyama M, Tanaka S, Yang R, Tanaka Y, et al. Comput Mater Sci 2006;36:281.

[473] Matsunaga K, Sasaki T, Shibata N, Mizoguchi T, Yamamoto T, Ikuhara Y. Phys Rev B 2006;74:125423.

[474] Shi S, Tanaka S, Kohyama M. Phys Rev B 2007;76:075431.

[475] Angelo JE, Moody NR, Baskes MI. Modell Simul Mater Sci Eng 1995;3:289.

[476] Geng WT, Freeman AJ, Wu R, Geller CB, Raynolds JE. Phys Rev B 1999;60:7149.

[477] Zhong LP, Wu RQ, Freeman AJ, Olson GB. Phys Rev B 2000;62:13938.

[478] Wang FH, Shang JX, Li JM, Wang CY. Intermetallics 2000;8:589.

[479] Liu LM, Wang SQ, Ye HQ. J Phys Condes Matter 2005;17:5335.

[480] Hao SQ, Delley B, Veprek S, Stampfl C. Phys Rev Lett 2006;97:086102.

[481] Chen J, Ouyang LZ, Rulis P, Misra A, Ching WY. Phys Rev Lett 2005;95:256103.

[482] Gleiter H. Acta Mater 2000;48:1.

[483] Valiev RZ, Islamgaliev RK, Alexandrov IV. Prog Mater Sci 2000;45:103.

[484] Kumar KS, Van Swygenhoven H, Suresh S. Acta Mater 2003;51:5743.

[485] Meyers MA, Mishra A, Benson DJ. Prog Mater Sci 2006;51:427.

[486] Dao M, Lu L, Asaro RJ, De Hosson JTM, Ma E. Acta Mater 2007;55:4041.

[487] Nix WD. Met Trans A Phys Met Mater Sci 1989;20:2217.

[488] Freund LB, Suresh S. Thin film materials: stress, defect formation and surface evolution. Cambridge: Cambridge University Press; 2004.

[489] Misra A, Kung H. Adv Eng Mater 2001;3:217.

[490] Misra A, Hirth JP, Hoagland RG. Acta Mater 2005;53:4817.

[491] Wang YM, Li J, Hamza AV, Barbee TW. Proc Natl Acad Sci USA 2007; 104:11155.

[492] Erlebacher J, Aziz MJ, Karma A, Dimitrov N, Sieradzki K. Nature 2001:410:450

[493] Volkert CA, Lilleodden ET, Kramer D, Weissmuller J. Appl Phys Lett 2006;89:061920.

[494] Hodge AM, Biener J, Hayes JR, Bythrow PM, Volkert CA, Hamza AV. Acta Mater 2007;55:1343.

[495] Wu B, Heidelberg A, Boland JJ. Nat Mater 2005;4:525.

[496] Han XD, Zheng K, Zhang YF, Zhang XN, Zhang Z, Wang ZL. Adv Mater 2007;19:2112.

[497] Uchic MD, Dimiduk DM, Florando JN, Nix WD. Science 2004;305:986.
[498] Volkert CA, Lilleodden ET. Philos Mag 2006;86:5567.

[499] Greer JR, Nix WD. Phys Rev B 2006;73:245410.

[500] Greer JR, Weinberger CR, Cai W. Mater Sci Eng A: Struct Mater Prop Microstruct Process 2008;493:21.

[501] Wang JL, Lian J, Greer JR, Nix WD, Kim KS. Acta Mater 2006;54:3973.

[502] Gerberich WW, Mook WM, Perrey CR, Carter CB, Baskes MI, Mukherjee R, et al. J Mech Phys Solids 2003;51:979.

[503] Shan ZW, Adesso G, Cabot A, Sherburne MP, Asif SAS, Warren OL, et al. Nat Mater 2008;7:947.

[504] Suresh S, Li J. Nature 2008;456:716

[505] Arzt E. Acta Mater 1998;46:5611.

[506] Yip S. Nature 1998;391:532.

[507] Van Swygenhoven H. Science 2002;296:66.

[508] Schiotz J, Jacobsen KW. Science 2003;301:1357.

[509] Yamakov V, Wolf D, Phillpot SR, Mukherjee AK, Gleiter H. Nat Mater 2004;3:43.

[510] Yip S. Nat Mater 2004;3:11.

[511] Van Swygenhoven H, Weertman JR. Mater Today 2006;9:24.

[512] Argon AS, Yip S. Philos Mag Lett 2006;86:713.

[513] Espinosa HD, Berbenni S, Panico M, Schwarz KW. Proc Natl Acad Sci USA 2005;102:16933.

[514] Rao SI, Dimiduk DM, Parthasarathy TA, Uchic MD, Tang M, Woodward C. Acta Mater 2008;56:3245.

[515] Derlet PM, Gumbsch P, Hoagland R, Li J, McDowell DL, van Swygenhoven H, et al. MRS Bull 2009;34:184.

[516] Hall EO. Proc Phys Soc Lond B 1951;64:747.

[517] Petch NJ. J Iron Steel Inst 1953;174:25.

[518] Amodeo RJ, Ghoniem NM. Phys Rev B 1990;41:6958.

[519] Zbib HM, Rhee M, Hirth JP. Int J Mech Sci 1998;40:113.

[520] Bulatov V, Abraham FF, Kubin L, Devincre B, Yip S. Nature 1998;391:669.

[521] Devincre B, Hoc T, Kubin L. Science 2008;320:1745.

[522] Weygand D, Poignant M, Gumbsch P, Kraft O. Mater Sci Eng A: Struct Mater Prop Microstruct Process 2008;483:188.

[523] Cai W, Bulatov VV, Chang JP, Li J, Yip S. In: Nabarro FRN, Hirth JP, editors. Dislocations in solids, vol. 12. Amsterdam: Elsevier; 2004. p. $1-80$.

[524] Rasmussen T, Vegge T, Leffers T, Pedersen OB, Jacobsen KW. Philos Mag A: Phys Condens Matter Struct Defect Mech Prop 2000;80:1273.

[525] Dimiduk DM, Woodward C, LeSar R, Uchic MD. Science 2006;312:1188.

[526] Csikor FF, Motz C, Weygand D, Zaiser M, Zapperi S. Science 2007;318:251.

[527] Eshelby JD, Frank FC, Nabarro FRN. Philos Mag 1951;42:351.

[528] Hirth JP. Philos Mag 2006;86:3959.

[529] Mughrabi H. Philos Mag 2006;86:4037.

[530] Frank FC, Read WT. Phys Rev 1950;79:722.

[531] Vitek V. Philos Mag 1968;18:773.

[532] Vitek V. Scripta Metall 1970;4:725.

[533] Van Swygenhoven H, Derlet PM, Froseth AG. Nat Mater 2004;3:399.

[534] Ogata S, Li J, Yip S. Phys Rev B 2005;71:224102.

[535] Warner DH, Sansoz F, Molinari JF. Int J Plast 2006;22:754.

[536] Valiev RZ, Alexandrov IV, Zhu YT, Lowe TC. J Mater Res 2002;17:5

[537] Wang YM, Ma E. Acta Mater 2004;52:1699.

[538] Zhu YT, Liao XZ. Nat Mater 2004;3:351.

[539] Hart EW. Acta Met 1967;15:351.

[540] Hutchinson JW, Neale KW. Acta Met 1977;25:839.

[541] Read WT, Shockley W. Phys Rev 1950;78:275.

[542] Lu L, Schwaiger R, Shan ZW, Dao M, Lu K, Suresh S. Acta Mater 2005;53:2169.

[543] Lu L, Chen X, Huang X, Lu K. Science 2009;323:607.

[544] Ogata S, Shimizu F, Li J, Wakeda M, Shibutani Y. Intermetallics 2006;14:1033

[545] Shimizu F, Ogata S, Li J. Mater Trans 2007;48:2923. 
[546] Schuh CA, Hufnagel TC, Ramamurty U. Acta Mater 2007;55:4067.

[547] Eshelby JD. Proc R Soc Lond Ser A Math 1957;241:376.

[548] Watanabe T, Kimura SI, Karashima S. Philos Mag A 1984;49:845.

[549] Broughton JQ, Gilmer GH. Phys Rev Lett 1986;56:2692.

[550] Suzuki A, Mishin Y. J Mater Sci 2005;40:3155.

[551] Keblinski P, Wolf D, Phillpot SR, Gleiter H. Philos Mag A 1999;79:2735.

[552] Frolov T, Mishin Y. Phys Rev B 2009;79:174110.

[553] Ubbelohde AR. Molten state of matter: melting and crystal structure. Chichester: Wiley; 1978.

[554] Luo J. Crit Rev Solid State Mater Sci 2007;32:67.

[555] Balluffi RW, Maurer R. Scripta Metall 1988;22:709.

[556] Hsieh TE, Balluffi RW. Acta Metall 1989;37:1637.

[557] Masumura RA, Glicksman ME, Vold CL. Scripta Metall 1972;6:943.

[558] Vold CL, Glicksman ME. In: Hu H, editor. The nature and behavior of grain boundaries. New York: Metallurgical Society of AIME/ Plenum Press; 1972. p. 171-83.

[559] Inoko F, Okada T, Muraga T, Nakano Y, Yoshikawa T. Interface Sci 1997;4:263.

[560] Divinski SV, Lohman M, Herzig C, Straumal B, Baretzky B, Gust W. Phys Rev B 2005;71:104104.

[561] Gupta VK, Yoon DH, Meyer HM, Luo J. Acta Mater 2007;55:3131.

[562] Bishop CM, Tang M, Cannon RM, Carter WC. Mater Sci Eng A 2006;422:102.

[563] Lobkovsky A, Warren JW. Physica D 2002;164:202.

[564] Tang M, Carter WC, Cannon RM. Phys Rev B 2006;73:024102.

[565] Tang M, Carter WC, Cannon RM. Phys Rev Lett 2006;97:075502.

[566] Mishin Y, Boettinger WJ, Warren JA, McFadden GB. Acta Mater 2009;57:3771.

[567] Berry J, Elder KR, Grant M. Phys Rev B 2008;77:224114.

[568] Mellenthin J, Karma A, Plapp M. Phys Rev B 2008;78:184110.

[569] Kikuchi R, Cahn JW. Phys Rev B 1980;21:1893.

[570] Besold G, Mouritsen OG. Comp Mater Sci 2000;18:225.

[571] Guillope M, Ciccotti G, Pontikis V. Surf Sci 1984;144:67.

[572] Broughton JQ, Gilmer GH. Modell Simul Mater Sci Eng 1998;6:87.

[573] Lutsko JF, Wolf D, Phillpot SR, Yip S. Phys Rev B 1989;40:2841.

[574] Nguyen T, Ho PS, Kwok T, Nitta C, Yip S. Phys Rev B 1992;46:6050.

[575] Lu J, Szpunar JA. Interface Sci 1995;3:143.

[576] Williams PL, Mishin Y. Acta Mater 2009;57:3786.

[577] von Alfthan S, Kaski K, Sutton AP. Phys Rev B 2007;76:245317.

[578] Frolov T, Mishin Y, in preparation.

[579] Tartaglino U, Zykova-Timan T, Ercolessi F, Tosatti E. Phys Rep 2005;411:291

[580] Wang NY, Rokhlin SI, Farson DF. Nanotechnology 2008;19:415701.

[581] Yang X, Hu W, Yuan X. Phys Lett A 2007;365:161.

[582] Sorkin V, Polturak E, Adler J. Phys Rev B 2003;68:174103.

[583] Dash JD, Fu HY, Weelaufer JS. Rep Prog Phys 1995;58:115.

[584] Lipowsky R. Phys Rev Lett 1986;57:2876.

[585] Lipowsky R, Fisher ME. Phys Rev B 1987;36:2126.

[586] Clarke DR. J Am Ceramic Soc 1987;70:15.

[587] Hoyt JJ, Olmsted D, Jindal S, Asta M, Karma A. Phys Rev E 2009;79:020601.

[588] Kaur I, Mishin Y, Gust W. Fundamentals of grain and interphase boundary diffusion. Chichester: Wiley; 1995.

[589] Mishin Y, Herzig Chr, Bernardini J, Gust W. Int Mater Rev 1997; $42: 155$

[590] Mishin Y, Herzig Chr. Mater Sci Eng A 1999;260:55.

[591] Liu CL, Plimpton SJ. Phys Rev B 1995;51:4523.

[592] Nomura M, Lee SY, Adams JB. J Mater Res 1991;6:1.

[593] Nomura M, Adams JB. J Mater Res 1992;7:3202.

[594] Nomura M, Adams JB. J Mater Res 1995;10:2916.

[595] Sørensen MR, Mishin Y, Voter AF. Phys Rev B 2000;62:3658.
[596] Suzuki A, Mishin Y. Interface Sci 2003;11:131.

[597] Suzuki A, Mishin Y. J Metastable Nonocryst Mater 2004;19:1.

[598] Schönfelder B, Gottstein G, Schvindlerman LS. Acta Mater 2005;53:1597.

[599] Pun GPP, Mishin Y, in preparation.

[600] Suzuki A, Mishin Y. Interface Sci 2003;11:425.

[601] Zhang H, Srolovitz DJ, Douglas JF, Warren JA. Acta Mater 2007;55:4527.

[602] Donati C, Douglas JF, Kob W, Plimpton SJ, Poole PH, Glotzer SC. Phys Rev Lett 1998;80:2338.

[603] Heesemann A, Zollmer V, Ratzke K, Faupel F. Phys Rev Lett 2000;84:1467.

[604] Frenkel J. Kinetic theory of liquids. New York: Dover; 1955.

[605] Mott NF. Proc Phys Soc 1948;60:391.

[606] Surholt T, Herzig Chr. Acta Mater 1997;45:3817.

[607] Pun GPP, Mishin Y. Defect Diffus Forum 2007;266:49.

[608] Pun GPP, Mishin Y. Acta Mater 2009;57:5531.

[609] Molodov DA, Konijnenberg PJ. Scripta Mater 2006;54:977.

[610] Bhaumik S, Molodova X, Molodov DA, Gottstein G. Scripta Mater 2006;55:995.

[611] Molodov DA, Bollmann C, Gottstein G. Mater Sci Eng A 2007;467:71.

[612] Gottstein G, Shvindlerman LS. Grain boundary migration in metals. Boca Raton, FL: CRC Press; 1999.

[613] Xiao S, Hu W. J Cryst Growth 2006;286:512.

[614] Upmanyu M, Srolovitz DJ, Schvindlerman LS, Gottstein G. Acta Mater 1999;47:3901.

[615] Upmanyu M, Srolovitz DJ, Shvindlerman LS, Gottstein G. Acta Mater 2002;50:1405.

[616] Zhang H, Upmanyu M, Srolovitz DJ. Acta Mater 2005;53:79.

[617] Schönfelder B, Wolf D, Phillpot SR, Furtkamp M. Interface Sci 1997;5:245.

[618] Zhang H, Mendelev MI, Srolovitz DJ. Acta Mater 2004;52:2569.

[619] Schönfelder B, Gottstein G, Schvindlerman LS. Metall Mater Trans A 2006;37:1757.

[620] Janssens KGF, Olmsted D, Holm EA, Foiles SM, Plimpton SJ, Derlet PM. Nat Mater 2006;5:124.

[621] Olmsted DL, Foiles SM, Holm EA. Scripta Mater 2007;57:1161.

[622] Trautt ZT, Upmanyu M, Karma A. Science 2006;314:632.

[623] Lobkovsky AE, Karma A, Medelev MI, Haataja M, Srolovitz DJ. Acta Mater 2004;52:285.

[624] Godiksen RB, Trautt ZT, Upmanyu M, Schiotz J, Jensen DJ, Schmidt S. Acta Mater 2007;55:6383.

[625] Godiksen RB, Schmidt S, Jensen DJ. Modell Simul Mater Sci Eng 2008;16:065002.

[626] Mendelev MI, Srolovitz DJ. Modell Simul Mater Sci Eng 2002;10:R79.

[627] Aoki M, Nguyen-Manh D, Pettifor DG, Vitek V. Prog Mater Sci 2007;52:154.

[628] Drautz R, Pettifor DG. Phys Rev B 2006;74:174117.

[629] Drautz R, Zhou XW, Murdick DA, Gillespie B, Wadley HNG, Pettifor DG. Prog Mater Sci 2007;52:196.

[630] Angioletti-Ubert S, Asta M, Finnis MW, Lee PD. Phys Rev B 2008;78:134203.

[631] Greeff CW. J Chem Phys 2008;128:184104.

[632] Mao Z, Sudbrack CK, Yoon KE, Martin G, Seidman DN. Nat Mater 2007;6:210.

[633] Seidman DN. MRS Bull 2009;34:537.

[634] Wang J, Wolverton C, Müller S, Liu ZK, Chen LQ. Acta Mater 2005;53:2759.

[635] Lill JV, Broughton JQ. Phys Rev Lett 2000;84:5784.

[636] Lill JV, Broughton JQ. Phys Rev B 2001;63:144102.

[637] Mendelev MI, Ackland GJ. Philos Mag Lett 2007;87:349.

[638] Dao M, Lu L, Shen Y, Suresh S. Acta Mater 2006;54:5421. 